JOURNAL OF THE

AMERICAN MATHEMATICAL SOCIETY

Volume 13, Number 1, Pages 1-54

S 0894-0347(99)00317-3

Article electronically published on August 23, 1999

\title{
TWO-PRIMARY ALGEBRAIC $K$-THEORY OF RINGS OF INTEGERS IN NUMBER FIELDS
}

\author{
J. ROGNES AND C. WEIBEL \\ WITH AN APPENDIX BY M. KOLSTER
}

\section{INTRODUCTION}

In the early 1970's, Lichtenbaum [L1, L2] made several distinct conjectures about the relation between the algebraic $K$-theory, étale cohomology and zeta function of a totally real number field $F$. This paper confirms Lichtenbaum's conjectural connection between the 2-primary $K$-theory and étale cohomology of $F$, and (when $\operatorname{Gal}(F / \mathbb{Q})$ is Abelian) to the zeta function. Up to a factor of $2^{r_{1}}$, we obtain the relationship conjectured by Lichtenbaum in [L2, 2.4 and 2.6]. In the special case $F=\mathbb{Q}$, this result was obtained in [W3].

Our methods depend upon the recent spectacular results of Voevodsky [V2], Suslin and Voevodsky [SV], and Bloch and Lichtenbaum [BL]. Together with Appendix B to this paper, they yield a spectral sequence starting with the étale cohomology of any field of characteristic zero and converging to its 2 -primary $K$-theory. For number fields, this is essentially the spectral sequence whose existence was conjectured by Quillen in [Q4]. The main technical difficulties with this spectral sequence, overcome in this paper, are that it does not degenerate at $E_{2}$ when $F$ has a real embedding, and that it has no known multiplicative structure.

To describe our result we introduce some notation. If $A$ is an Abelian group, we let $A\{2\}$ denote its 2-primary torsion subgroup, and let \#A denote its order when $A$ is finite. We write $K_{n}(R)$ for the $n$th algebraic $K$-group of a $\operatorname{ring} R$, and $H_{\text {ét }}^{n}(R ; M)$ for the $n$th étale cohomology group of $\operatorname{Spec}(R)$ with coefficients in $M$.

Theorem 0.1. Let $F$ be a totally real number field, with $r_{1}$ real embeddings. Let $R=\mathcal{O}_{F}\left[\frac{1}{2}\right]$ denote the ring of 2 -integers in $F$. Then for all even $i>0$

$$
2^{r_{1}} \cdot \frac{\# K_{2 i-2}(R)\{2\}}{\# K_{2 i-1}(R)\{2\}}=\frac{\# H_{\text {ét }}^{2}\left(R ; \mathbb{Z}_{2}(i)\right)}{\# H_{\text {ét }}^{1}\left(R ; \mathbb{Z}_{2}(i)\right)} \text {. }
$$

(It is well known that all $K$ - and cohomology groups appearing in this formula are finite.)

This theorem is an immediate consequence of Theorem 0.6 below. It can also be formulated in terms of the $K$-theory of the $\operatorname{ring} \mathcal{O}_{F}$ of integers in $F$, because the localization sequence implies that $K_{n}\left(\mathcal{O}_{F}\right)\{2\} \cong K_{n}(R)\{2\}$ for all $n>0$.

Received by the editors July 13, 1998.

2000 Mathematics Subject Classification. Primary 19D50; Secondary 11R70, 11S70, 14F20, $19 \mathrm{~F} 27$.

Key words and phrases. Two-primary algebraic $K$-theory, number fields, Lichtenbaum-Quillen conjectures, étale cohomology, motivic cohomology, Bloch-Lichtenbaum spectral sequence. 
Lichtenbaum made a second conjecture relating the values $\zeta_{F}(1-i)$ of the zeta function to étale cohomology (and also to $K$-theory); see [L1, 9.1] and [L2, 1.5]. The $\ell \neq 2$ part of this conjecture was established by Wiles in 1990; see Theorem 1.6 of [Wi]. We have included an appendix to this paper, written by M. Kolster, showing that the 2-primary part of Lichtenbaum's conjecture is a consequence of the 2-adic Main Conjecture of Iwasawa theory for the trivial character. The appendix also explains how a theorem of Wiles (see [Wi, Gri]) implies that this Main Conjecture holds for all totally real Abelian number fields. Let the notation $a \sim_{2} b$ indicate that the rational numbers $a$ and $b$ have the same 2 -adic valuation. We have then verified Lichtenbaum's second conjecture for such fields:

Theorem 0.2. Let $F$ be a totally real Abelian number field, and $R$ its ring of 2integers. Then for all even $i>0$

$$
\zeta_{F}(1-i) \quad \sim_{2} \quad 2^{r_{1}} \cdot \frac{\# K_{2 i-2}(R)\{2\}}{\# K_{2 i-1}(R)\{2\}} .
$$

The problem of computing the higher $K$-theory of a number field $F$, or rather of its ring of 2-integers $R=\mathcal{O}_{F}\left[\frac{1}{2}\right]$, has a rich history. The groups $K_{n}(R)$ are finitely generated by [Q3], and we have already mentioned Lichtenbaum's conjectures about their orders. Harris and Segal showed in [HS] that each $K_{2 i-1}\left(\mathcal{O}_{F}\right)$ contains a cyclic summand whose 2-order is $w_{i}(F), 2 w_{i}(F)$ or $w_{i}(F) / 2$. Here the numbers $w_{i}(F)$ are defined as the maximal powers of 2 such that $\operatorname{Gal}\left(F\left(\zeta_{2^{n}}\right) / F\right)$ has exponent dividing $i$, and can be calculated easily in particular cases; see Definition 1.7 and Proposition 1.9.

Our results resolve the indeterminacy in the order of these "Harris-Segal" summands. By [HS], the indeterminacy is only an issue when $F$ is "exceptional," and was eliminated or reduced in several cases in [W1]. The following statement is extracted from Theorems 1.13 and 6.14 .

Theorem 0.3. Let $F$ be a number field, and set $w_{i}=w_{i}(F)$. When $F$ is totally imaginary, the 2-primary Harris-Segal summand in $K_{2 i-1}\left(\mathcal{O}_{F}\right)$ is cyclic of order $w_{i}$. When $F$ has a real embedding, then the 2-primary Harris-Segal summand is: $\mathbb{Z} / w_{i}$ when $i \equiv 0,1 \bmod 4 ; \mathbb{Z} / 2 w_{i}$ when $i \equiv 2 \bmod 4$; and is zero when $i \equiv 3 \bmod 4$.

Historically, the next breakthrough was Soulé's thesis [So], which showed the importance of $K$-theory with coefficients, and established some parts of Lichtenbaum's conjectures for $\ell \neq 2$.

Since 1980 , most of the work on the $K$-theory of number fields has focussed upon Quillen's conjecture in [Q4] that there should be an Atiyah-Hirzebruch type spectral sequence which starts at the étale cohomology of $R$, and has an abutment coinciding with the $\ell$-adic $K$-theory of $R$ in degrees $\geq 2$. (Here $R$ is still the ring of 2-integers in a number field $F$.) The next significant step was taken by Dwyer and Friedlander $[\mathrm{DF}]$; they constructed the "étale $K$-theory" of a scheme, which is naturally equipped with an Atiyah-Hirzebruch type spectral sequence as well as a comparison map $K_{*}(R) \otimes \mathbb{Z}_{\ell} \rightarrow \hat{K}_{n}^{\text {ét }}(R)$. They proved that the comparison map is onto in degrees $n \geq 1$, assuming either that $\ell \neq 2$ or that $\sqrt{-1} \in F$.

We will not attempt to mention all the work on the Lichtenbaum-Quillen conjectures in the last 20 years (Math. Reviews lists over 50 papers on the subject), mentioning only a few of the authors not named above: Alexander Beilinson, Bruno Kahn, Steve Mitchell, Vic Snaith and Bob Thomason. The best results to date have been for odd primes, because of the following technical problem that occurs when 
$\ell=2$ : the Dwyer-Friedlander spectral sequence of a number field $F$ is not bounded, and so is not known to converge, unless $F$ is totally imaginary. In addition, its multiplicative structure is less useful when $\sqrt{-1} \notin F$.

Using the results of Voevodsky [V2] and Bloch-Lichtenbaum [BL], we refine the Dwyer-Friedlander results, and extend them to all totally imaginary number fields. The result, as well as the methods we use, were suggested by Quillen in [Q4].

Theorem 0.4. Let $R=\mathcal{O}_{F}\left[\frac{1}{2}\right]$ be the ring of 2-integers in any totally imaginary number field $F$. (a) For all $n \geq 2$

$$
K_{n}(R) \cong \begin{cases}H_{\text {ét }}^{2}\left(R ; \mathbb{Z}_{2}(i+1)\right) \oplus(\text { odd }) & \text { for } n=2 i, \\ \mathbb{Z}^{r_{2}} \oplus \mathbb{Z} / w_{i}(F) \oplus(\text { odd }) & \text { for } n=2 i-1 .\end{cases}
$$

Here the notation "(odd)" refers to a finite group of odd order.

(b) Suppose in addition that $\sqrt{-1} \in F$. Then the natural map induces an isomorphism

$$
K_{n}(R) \otimes \mathbb{Z}_{2} \cong \hat{K}_{n}^{\text {ét }}(R)
$$

for $n \geq 1$.

Part (a) of this theorem will be proven as Theorem 6.14(a) below. Note that it can also be written in terms of $\mathcal{O}_{F}$ because if $n \geq 2$, then $K_{n}\left(\mathcal{O}_{F}\right)$ is a subgroup of finite odd index in $K_{n}(R)$. Part (b) will be extended to all totally imaginary $F$ in $[\mathrm{RW}]$.

Proof of part (b). Dwyer and Friedlander proved in [DF, 8.7] that the natural map $K_{n}(R) \otimes \mathbb{Z}_{2} \rightarrow \hat{K}_{n}^{\text {ét }}(R)$ is a surjection for all $n \geq 1$. Part (a) combined with [DF, 8.2 and 8.8], shows that the two sides are abstractly isomorphic as finitely generated $\mathbb{Z}_{2}$-modules for $n \geq 1$. Hence the natural map is an isomorphism when $n \geq 1$.

Here is the local analog of Theorem 0.4; it will be proven as Theorem 3.7 below. Recall that a $p$-local field $E$ of characteristic zero is a finite extension of the field $\mathbb{Q}_{p}$ of $p$-adic numbers. By considering the 2 -adically completed $K$-groups $K_{n}\left(E ; \mathbb{Z}_{2}\right)$ of $E$ we avoid accounting for the typically uncountable uniquely divisible summands in $K_{n}(E)$.

Theorem 0.5. Let $E$ be a 2-local field of characteristic zero with valuation ring $\mathcal{O}_{E}$, and set $d=\left[E: \mathbb{Q}_{2}\right]$. Then for all $n \geq 2$, the 2-adically completed $K$-groups of $\mathcal{O}_{E}$ and $E$ are:

$$
K_{n}\left(\mathcal{O}_{E} ; \mathbb{Z}_{2}\right) \cong K_{n}\left(E ; \mathbb{Z}_{2}\right) \cong \begin{cases}\mathbb{Z} / w_{i}(E) & \text { for } n=2 i, \\ \mathbb{Z}_{2}^{d} \oplus \mathbb{Z} / w_{i}(E) & \text { for } n=2 i-1 .\end{cases}
$$

As we have mentioned, our methods yield a concrete calculation of the 2-torsion in the algebraic $K$-theory of the ring of integers in a number field. When $F$ has a real embedding there is a kind of periodicity of order eight, related to the 8-fold periodicity in the homotopy groups of $B O$ and the image of $J$. To describe it, we use the traditional notation that $r_{1}$ and $r_{2}$ denote the number of real, resp. pairs, of complex embeddings of $F$. 
We write $A \rtimes B$ for an Abelian group extension of $B$ by $A$, i.e., an Abelian group containing $A$ with quotient $B$.

Theorem 0.6. Let $F$ be a number field with at least one real embedding, and let $R=\mathcal{O}_{F}\left[\frac{1}{2}\right]$ denote the ring of 2-integers in $F$. Then for all $n \geq 2$

$$
K_{n}\left(\mathcal{O}_{F}\right) \cong K_{n}(R) \cong \begin{cases}H_{\text {ét }}^{2}\left(R ; \mathbb{Z}_{2}(4 k+1)\right) & \text { for } n=8 k, \\ \mathbb{Z}^{r_{1}+r_{2}} \oplus \mathbb{Z} / 2 & \text { for } n=8 k+1, \\ H_{\text {ét }}^{2}\left(R ; \mathbb{Z}_{2}(4 k+2)\right) & \text { for } n=8 k+2, \\ \mathbb{Z}^{r_{2}} \oplus(\mathbb{Z} / 2)^{r_{1}-1} \oplus \mathbb{Z} / 2 w_{4 k+2}(F) & \text { for } n=8 k+3, \\ (\mathbb{Z} / 2)^{\rho} \rtimes H_{\text {ét }}^{2}\left(R ; \mathbb{Z}_{2}(4 k+3)\right) & \text { for } n=8 k+4, \\ \mathbb{Z}^{r_{1}+r_{2}} & \text { for } n=8 k+5, \\ \tilde{H}_{\text {ét }}^{2}\left(R ; \mathbb{Z}_{2}(4 k+4)\right) & \text { for } n=8 k+6, \\ \mathbb{Z}^{r_{2}} \oplus \mathbb{Z} / w_{4 k+4}(F) & \text { for } n=8 k+7,\end{cases}
$$

modulo odd finite groups. Here $j \leq \rho<r_{1}$, where $j$ is the signature defect of $R$ (see Theorem 0.7 and Definition 7.3), and $\tilde{H}_{\text {èt }}^{2}\left(R ; \mathbb{Z}_{2}(4 k+4)\right.$ ) is the kernel of the natural surjective map

$$
\alpha^{2}: H_{\text {êt }}^{2}\left(R ; \mathbb{Z}_{2}(4 k+4)\right) \rightarrow \bigoplus^{r_{1}} H_{\text {êt }}^{2}\left(\mathbb{R} ; \mathbb{Z}_{2}(4 k+4)\right) \cong(\mathbb{Z} / 2)^{r_{1}}
$$

induced by the $r_{1}$ real embeddings of $F$ (see Definition 6.6).

The 2-primary subgroup of the finite group $K_{8 k+4}(R)$ is isomorphic to the maximal finite quotient of $K_{8 k+5}\left(R ; \mathbb{Z} / 2^{\infty}\right)$. The latter is $K$-theory with coefficients $\mathbb{Z} / 2^{\infty}$, and it fits into the short exact sequence

$$
0 \rightarrow(\mathbb{Z} / 2)^{r_{1}-1} \rightarrow K_{8 k+5}\left(R ; \mathbb{Z} / 2^{\infty}\right) \rightarrow\left(\mathbb{Z} / 2^{\infty}\right)^{r_{1}+r_{2}} \oplus H_{\text {èt }}^{2}\left(R ; \mathbb{Z}_{2}(4 k+3)\right) \rightarrow 0 .
$$

We prove this result as Theorem 6.14(b), using Borel's calculation (cited as Theorem 6.2 below) of the rational ranks of these groups. The extensions in degrees $8 k+3$ and $8 k+4$, as well as the inequality $j \leq \rho<r_{1}$, are discussed in Corollary 7.12 .

Simple examples of totally real fields with $r_{1}=2$ such that $K_{8 k+4}(R)$ has no 2torsion (so $\rho=j=0$ ) are given by Rognes and Østvær in [RO]. One such example is $F=\mathbb{Q}(\sqrt{5}), R=\mathbb{Z}\left[\sqrt{5}, \frac{1}{2}\right]$.

While the exact structure of the even $K$-groups $K_{2 i-2}(R)$ remains as intricate as the étale cohomology groups $H_{\text {ét }}^{2}\left(R ; \mathbb{Z}_{2}(i)\right)$, we do know the 2-rank of these $K$-groups. See Definition 7.3 for a discussion of the Picard group $\operatorname{Pic}(R)$ and the narrow Picard group $\mathrm{Pic}_{+}(R)$ appearing in the following result, which will be proven as Theorem 7.11.

Theorem 0.7. Let $F$ be a number field with $r_{1}>0$ real embeddings and $r_{2} \geq 0$ pairs of complex embeddings. Let $R$ be its ring of 2-integers, let $s$ be the number of prime ideals in $\mathcal{O}_{F}$ dividing 2 , let $t$ be the 2-rank of the Picard group $\operatorname{Pic}(R)$, and let $u$ be the 2-rank of the narrow Picard group $\mathrm{Pic}_{+}(R)$. (The signature defect of $R$ 
is $j=u-t$.) Then for all $n>0$

$$
\text { the 2-rank of } K_{n}(R)\{2\}= \begin{cases}s+t-1 & \text { for } n=8 k, \\ 1 & \text { for } n=8 k+1, \\ r_{1}+s+t-1 & \text { for } n=8 k+2, \\ r_{1} & \text { for } n=8 k+3, \\ s+u-1 & \text { for } n=8 k+4, \\ 0 & \text { for } n=8 k+5, \\ s+u-1 & \text { for } n=8 k+6, \\ 1 & \text { for } n=8 k+7 .\end{cases}
$$

The main part of our work uses the Bloch-Lichtenbaum spectral sequence of [BL] with $\mathbb{Z} / 2^{\infty}$-coefficients to compute the algebraic $K$-groups $K_{*}\left(R ; \mathbb{Z} / 2^{\infty}\right)$. The higher Chow groups describing the $E_{2}$-term of this spectral sequence were reexpressed as motivic cohomology groups by Suslin in [S2], and as étale cohomology groups by Suslin and Voevodsky in [SV], using Voevodsky's results in [V2].

In section 1 we discuss a mod $2^{\nu}$ version of the Bloch-Lichtenbaum spectral sequence and handle the field versions of Theorems 0.4 and 0.5. In sections 2 and 4 we review the portions of étale cohomology theory we need. We prove Theorem 0.5 in section 3. Section 5 is devoted to the Bloch-Lichtenbaum spectral sequence for the real numbers. Our calculation of $K_{*}\left(R ; \mathbb{Z} / 2^{\infty}\right)$ is given in section 6 . We then obtain the information about the 2-adic $K$-groups $K_{*}\left(R ; \mathbb{Z}_{2}\right) \cong K_{*}(R) \otimes \mathbb{Z}_{2}$ using universal coefficient theorems such as Proposition 2.4. The number of summands in $K_{2 i}(R)\{2\}$ is determined in section 7 , along with the $K$-theory $K_{*}(R ; \mathbb{Z} / 2)$ with coefficients mod 2 .

We would like to point out that we do not make any assumption that the BlochLichtenbaum spectral sequence behaves well with respect to multiplication by either the Hopf map $\eta$ or the Bott element $\beta$. That assumption remains unverified, although it is conjectured to hold by Beilinson, and was used in the papers [W3] and [K2] in order to obtain results similar to our Theorems 0.1, 0.2 and 0.6. We circumvent this difficulty in section 5 , using topological information about $K\left(\mathbb{Q}_{2}\right)$ from $[\mathrm{R}]$. In particular, Theorem 5.3 below provides a substitute for Proposition 4 in [W3]; this substitution justifies the calculation of the 2-torsion in $K_{*}(\mathbb{Z})$ in [W3]. Many of the results in Bruno Kahn's preprint [K2] can also be justified by this result.

This paper evolved over twelve months, beginning with the Oberwolfach conference in June 1996. We completed the mod 2 and 2-adic calculations in July and September 1996, respectively. However, our calculations depended upon the assumption that the differentials in the Bloch-Lichtenbaum spectral sequence for $\mathbb{R}$ commute with multiplication by the Hopf map $\eta$, an assumption we thought we could easily prove. Meanwhile Kahn's preprint [K2] appeared in January 1997, proving the results under the assumption that the spectral sequence commutes with both $\eta$ and $\beta$. His preprint clarified our thinking in several respects, especially with respect to the unknown quantity $\rho$ in Theorem 0.6 , and we would like to thank him for useful discussions in the following months.

The authors would also like to thank Vladimir Voevodsky for his encouragement and his hints. Weibel thanks Spencer Bloch, Steve Lichtenbaum and Mark Walker for helpful discussions about [BL], [L1] and motivic cohomology. We also 
thank Manfred Kolster for writing the appendix on zeta-functions and the Main Conjecture of Iwasawa theory.

\section{The Bloch-Lichtenbaum spectral sequence}

In order to compute the 2-primary part of the algebraic $K$-theory of a field $F$, we shall use a certain spectral sequence. We begin by describing it.

In [BL, 1.3.4], Bloch and Lichtenbaum constructed a third quadrant spectral sequence converging to the algebraic $K$-groups of any field $F$ :

$$
E_{2}^{p, q}=C H^{-q}(F,-p-q) \Longrightarrow K_{-p-q}(F) .
$$

Here the $C H^{i}(F, n)$ denote Bloch's higher Chow groups of the scheme $\operatorname{Spec}(F)$. By definition (see [Bl]), $C H^{i}(F, n)$ is the $n$th homotopy group of a simplicial Abelian group $[p] \mapsto \mathcal{Z}_{\mathrm{Bl}}^{i}(F, p)$, which is free Abelian in each degree and zero in degrees $p<i$. In particular, $C H^{i}(F, n)=0$ for $n<i$ and $E_{2}^{p, q}=0$ for $p>0$. The terms along the $q$-axis are isomorphic to Milnor $K$-theory: $C H^{i}(F, i) \cong K_{i}^{M}(F)$. This is a result of Nesterenko and Suslin [NS, 4.9].

In fact, their construction also induces a spectral sequence with any finite coefficients $\mathbb{Z} / m$. Unfortunately, one may not derive this finite coefficient variant by a naïve modification of the arguments in [BL], because a crucial step (Corollary 2.3.3, used in the "key step" of [BL, 6.1]) fails to hold with finite coefficients. We have provided a correct derivation of the finite coefficient spectral sequence in Appendix B. Defining $C H^{i}(F, n ; \mathbb{Z} / m)$ as the $n$th homotopy group of $\mathcal{Z}_{\mathrm{Bl}}^{i}(F, \cdot) \otimes \mathbb{Z} / m=\mathcal{Z}_{\mathrm{Bl}}^{i}(F, \cdot ; \mathbb{Z} / m)$, the third quadrant spectral sequence associated to the exact couple becomes

$$
E_{2}^{p, q}=C H^{-q}(F,-p-q ; \mathbb{Z} / m) \Longrightarrow K_{-p-q}(F ; \mathbb{Z} / m) .
$$

Note that again $E_{2}^{p, q}=0$ for $p>0$ and that the terms along the $q$-axis are now $C H^{i}(F, i ; \mathbb{Z} / m) \cong K_{i}^{M}(F) \otimes \mathbb{Z} / m$.

It remains to translate the $E_{2}$-terms of this spectral sequence into étale cohomology groups. We do this in two steps, the first step being a straightforward translation into the language of motivic cohomology. Let $H_{\mathcal{M}}^{n}\left(F ; \mathbb{Z} / \ell^{\nu}(i)\right)$ denote the motivic cohomology groups of $\operatorname{Spec}(F)$ as defined in [S3], [FV] and [V1]. Up to vocabulary, Suslin proved in [S2] that these motivic cohomology groups agree with Bloch's higher Chow groups with finite coefficients, when $F$ is of characteristic zero. Here is a more precise statement.

Proposition 1.1 (Suslin). Let $X$ be a smooth affine variety over a field $F$ of characteristic zero. Then there are natural isomorphisms

$$
C H^{i}(X, n) \cong H_{\mathcal{M}}^{2 i-n}(X ; \mathbb{Z}(i)) \quad \text { and } \quad C H^{i}\left(X, n ; \mathbb{Z} / \ell^{\nu}\right) \cong H_{\mathcal{M}}^{2 i-n}\left(X ; \mathbb{Z} / \ell^{\nu}(i)\right) .
$$

Proof. Following $[\mathrm{FV}]$, let $\mathcal{Z}_{\text {equi }}(Y, r)(U)$ denote the free Abelian group on the integral subschemes $Z$ of $Y \times U$ which are equidimensional of relative dimension $r$ over $U$. Letting $U$ run over the cosimplicial scheme $\Delta^{\prime}$ yields a simplicial Abelian group which we write (abusively but simply) as $\mathcal{Z}_{\text {equi }}(Y, r)$. By [FV] 9.2, 8.2 and 8.1 (which require characteristic zero), we have:

$$
H_{\mathcal{M}}^{2 i-n}(X ; \mathbb{Z}(i)) \cong A_{0, n}\left(X, \mathbb{A}^{i}\right) \cong A_{d, n}\left(F, X \times \mathbb{A}^{i}\right) \cong \pi_{n} \mathcal{Z}_{\text {equi }}\left(X \times \mathbb{A}^{i}, d\right) .
$$

Here $d$ is the dimension of $X$, so that the subschemes $Z$ in $X \times \mathbb{A}^{i} \times U$ have codimension $i$. By $[\mathrm{S} 2,2.1]$ and homotopy invariance of Bloch's higher Chow groups 
[Bl], we have

$$
\pi_{n} \mathcal{Z}_{\text {equi }}\left(X \times \mathbb{A}^{i}, d\right) \cong C H^{i}\left(X \times \mathbb{A}^{i}, n\right) \cong C H^{i}(X, n) .
$$

The variant with coefficients is immediate.

The étale cohomology groups $H_{\text {ét }}^{n}(\operatorname{Spec}(R) ; M)$ are defined when $R$ is a commutative unital ring, and $M$ is an étale sheaf on $\operatorname{Spec}(R)$. Milne's book [M1] is a general reference for étale cohomology.

To complete the translation into étale cohomology we need two results. Voevodsky proved in [V2] that the Galois symbol $K_{n}^{M}(F) / 2 \rightarrow H_{\text {ét }}^{n}(F ; \mathbb{Z} / 2)$ is an isomorphism for every field $F$ of characteristic $\neq 2$, and every $n \geq 0$. Suslin and Voevodsky showed in [SV, 5.11] that for $F$ a field of characteristic zero this implies (among other things) that there is a natural isomorphism

$$
H_{\mathcal{M}}^{n}(F ; \mathbb{Z} / 2(i)) \cong \begin{cases}H_{\text {ét }}^{n}(F ; \mathbb{Z} / 2(i)) & \text { for } 0 \leq n \leq i \\ 0 & \text { otherwise. }\end{cases}
$$

Here the étale sheaf $\mathbb{Z} / 2(i)$ is just the constant sheaf $\mathbb{Z} / 2$. This is independent of $i$, since there are no nontrivial module actions upon the Abelian group $\mathbb{Z} / 2$. We shall use the additive notation $\mathbb{Z} / 2^{\nu}(i)$ for the étale sheaf $\mu_{2^{\nu}}^{\otimes i}$ (see Notations 2.3).

There is a natural map $H_{\mathcal{M}}^{n}\left(F ; \mathbb{Z} / 2^{\nu}(i)\right) \rightarrow H_{\text {ét }}^{n}\left(F ; \mathbb{Z} / 2^{\nu}(i)\right)$ for all $\nu(c f$. [SV, $5.3])$, so a 5-lemma argument shows that more generally

$$
H_{\mathcal{M}}^{n}\left(F ; \mathbb{Z} / 2^{\nu}(i)\right) \cong \begin{cases}H_{\text {ét }}^{n}\left(F ; \mathbb{Z} / 2^{\nu}(i)\right) & \text { for } 0 \leq n \leq i \\ 0 & \text { otherwise. }\end{cases}
$$

Hence when $F$ has characteristic zero and we are working with 2-primary coefficients, the $E_{2}$-term of the Bloch-Lichtenbaum spectral sequence may be expressed as:

$$
E_{2}^{p, q}=\left\{\begin{array}{ll}
H_{\text {ét }}^{p-q}\left(F ; \mathbb{Z} / 2^{\nu}(-q)\right) & \text { for } q \leq p \leq 0, \\
0 & \text { otherwise }
\end{array} \Longrightarrow K_{-p-q}\left(F ; \mathbb{Z} / 2^{\nu}\right) .\right.
$$

Passing to the colimit over $\nu$, and writing $W(i)$ for the union of the étale sheaves $\mathbb{Z} / 2^{\nu}(i)$, we also obtain a spectral sequence:

$$
E_{2}^{p, q}= \begin{cases}H_{\text {ét }}^{p-q}(F ; W(-q)) & \text { for } q \leq p \leq 0, \Longrightarrow K_{-p-q}\left(F ; \mathbb{Z} / 2^{\infty}\right) . \\ 0 & \text { otherwise }\end{cases}
$$

Hereafter we briefly denote étale cohomology groups by $H^{n}(R ; M)=H_{\text {ét }}^{n}(R ; M)$.

It is not so clear whether there is an inverse limit spectral sequence, with 2adic coefficients, since the cohomology groups of fields are typically not finitely generated, and limits of such groups are typically not exact.

To summarize:

Theorem 1.4. For each field $F$ of characteristic zero there are spectral sequences (1.2) and (1.3) as above, which are natural in $F$ and in the coefficients. We call these the $\bmod 2^{\nu}$ and $\bmod 2^{\infty}$ Bloch-Lichtenbaum spectral sequences for $F$, respectively.

Remark 1.5. If the Dwyer-Friedlander spectral sequence in $[\mathrm{DF}]$ is reindexed, it looks like the Bloch-Lichtenbaum spectral sequence (1.2) with the restriction $p \leq 0$ lifted. We suspect that the Bloch-Lichtenbaum spectral sequence agrees with the reindexed Dwyer-Friedlander spectral sequence for $p \leq 0$, when $\operatorname{cd}_{2}(F)<\infty$. This 
seems particularly likely in light of our Theorem $0.4(\mathrm{~b})$. If so, we would be able to use multiplicative tricks like [DF, 5.4], when $\operatorname{cd}_{2}(F)<\infty$. See [K1].

Example 1.6. When $\bar{F}$ is algebraically closed, e.g. $\bar{F}=\mathbb{C}$, then $H^{n}(\bar{F} ; W(i))=0$ for $n \neq 0$ and $H^{0}(\bar{F} ; W(i))=\mathbb{Z} / 2^{\infty}$ for all $i$. Thus (if $\operatorname{char}(F)=0$ ) the $\bmod 2^{\infty}$ spectral sequence collapses at the $E_{2}$-term. We recover

$$
K_{n}\left(\bar{F} ; \mathbb{Z} / 2^{\infty}\right) \cong \begin{cases}\mathbb{Z} / 2^{\infty} & \text { for } n \text { even, } \\ 0 & \text { for } n \text { odd. }\end{cases}
$$

In the $\bmod 2$ case, $H^{n}(\bar{F} ; \mathbb{Z} / 2)=0$ for $n \neq 0$ and $H^{0}(\bar{F} ; \mathbb{Z} / 2)=\mathbb{Z} / 2$, so the $\bmod 2$ spectral sequence also collapses at the $E_{2}$-term, with

$$
K_{n}(\bar{F} ; \mathbb{Z} / 2) \cong \begin{cases}\mathbb{Z} / 2 & \text { for } n \text { even } \\ 0 & \text { for } n \text { odd }\end{cases}
$$

Example 1.6 shows that the edge map

$$
e: K_{2 i}\left(F ; \mathbb{Z} / 2^{\infty}\right) \rightarrow H^{0}(F ; W(i)) \subseteq W(i)
$$

in the $\bmod 2^{\infty}$ Bloch-Lichtenbaum spectral sequence (1.3) has an elementary interpretation. It factors as the projection of $K_{2 i}\left(F ; \mathbb{Z} / 2^{\infty}\right)$ onto the 2-torsion subgroup $K_{2 i-1}(F)\{2\}$ of $K_{2 i-1}(F)$, followed by the natural map from $K_{2 i-1}(F)\{2\}$ to $K_{2 i-1}(\bar{F})\{2\} \cong W(i)$. Following [W1, p. 276], we shall call $e$ the (2-primary) $e$-invariant, since by [Q5] it is a generalization of Adams' complex $e$-invariant. The size of the target group $H^{0}(F ; W(i))$ is determined by the presence of roots of unity in cyclotomic extensions, as follows.

Definition 1.7. Fix a prime $\ell$ and let $F$ be a field. We define an integer $w_{i}(F)$ by

$$
w_{i}(F)=\max \left\{\ell^{\nu} \mid \operatorname{Gal}\left(F\left(\mu_{\ell^{\nu}}\right) / F\right) \text { has exponent dividing } i\right\}
$$

for each integer $i$. If there is no maximum we let $w_{i}(F)=\ell^{\infty}$. In particular $\mathbb{Z} / w_{0}(F)=\mathbb{Z} / \ell^{\infty}$. Note that $w_{-i}(F)=w_{i}(F)$ for all $i$.

We call a field $F$ exceptional if the Galois group $\operatorname{Gal}\left(F\left(\mu_{\ell^{\nu}}\right) / F\right)$ is not cyclic for some $\nu$, and nonexceptional otherwise. There are exceptional fields only for $\ell=2$. Real number fields are exceptional when $\ell=2$.

Proposition 1.8. Let $F$ be any field. Then $H^{0}(F ; W(i)) \cong \mathbb{Z} / w_{i}(F)$.

Proof. Let $\zeta$ be a primitive $\ell^{\nu}$ th root of unity. Then $\zeta^{\otimes i}$ is invariant under $g \in G_{F}$ (the absolute Galois group) precisely when $g^{i} \zeta=\zeta$, and $\zeta^{\otimes i}$ is invariant under all of $G_{F}$ precisely when the group $\operatorname{Gal}\left(F\left(\mu_{\ell^{\nu}}\right) / F\right)$ has exponent $i$.

We recall the following from $[\mathrm{W} 1,6.3]$. (See $[\mathrm{HS}, \mathrm{p} .28]$ when $\ell$ is odd.) Let $\log _{\ell}(n)$ be the maximal power of $\ell$ dividing $n$, i.e., the $\ell$-adic valuation of $n$. By convention let $\log _{\ell}(0)=\infty$.

Proposition 1.9. Let $F$ be a field of characteristic $\neq 2$. Let a be maximal such that $F(\sqrt{-1})$ contains a primitive $2^{a}$ th root of unity, i.e., $\mu_{2^{\infty}}(F(\sqrt{-1}))=\mu_{2^{a}}$. Let $i$ be any integer, and let $b=\log _{2}(i)$.

(a) If $\sqrt{-1} \in F$, then $w_{i}(F)=2^{a+b}$.

(b) If $\sqrt{-1} \notin F$ and $i$ is odd, then $w_{i}(F)=2$.

(c) If $\sqrt{-1} \notin F, F$ is exceptional and $i$ is even, then $w_{i}(F)=2^{a+b}$.

(d) If $\sqrt{-1} \notin F, F$ is nonexceptional and $i$ is even, then $w_{i}(F)=2^{a+b-1}$. 
For example, $w_{i}(\mathbb{R})$ is 2 if $i$ is odd, and $2^{\infty}$ if $i$ is even. In this case it is well known that the $e$-invariant is onto for $i$ even, and has image $\mathbb{Z} / 2$ for $i \equiv 1 \bmod 4$. In section 5 we will consider the rest of the $\bmod 2^{\infty}$ spectral sequence for the field of real numbers. This will allow us to treat real number fields in sections 6 and 7 ; cf. Theorems 6.7 and 7.4 .

Although it does not have characteristic zero, the $e$-invariant of a finite field is well known to be an isomorphism. For later reference, we record the Galois cohomology of $\mathbb{F}_{q}$ (taken from [So, III.1.4]) and the $K$-groups of $\mathbb{F}_{q}$ (taken from [Q1]).

Proposition 1.10. Let $\mathbb{F}_{q}$ be the finite field with $q$ elements. Then $w_{i}\left(\mathbb{F}_{q}\right)$ is the greatest power of $\ell$ dividing $q^{i}-1$, and

$$
K_{n}\left(\mathbb{F}_{q}\right)= \begin{cases}\mathbb{Z} & \text { for } n=0 \\ \mathbb{Z} /\left(q^{i}-1\right) & \text { for } n=2 i-1>0 \\ 0 & \text { otherwise. }\end{cases}
$$

Suppose $(\ell, q)=1$. When $i \neq 0$ we have $H^{n}\left(\mathbb{F}_{q} ; W(i)\right)=0$ for $n \neq 0$, and $H^{0}\left(\mathbb{F}_{q} ; W(i)\right)=\mathbb{Z} / w_{i}\left(\mathbb{F}_{q}\right)$. When $i=0$ we have $H^{n}\left(\mathbb{F}_{q} ; W(0)\right) \cong \mathbb{Z} / \ell^{\infty}$ for $n=$ 0,1 , and $H^{n}\left(\mathbb{F}_{q} ; W(0)\right)=0$ for $n \neq 0,1$. In nonnegative degrees

$$
K_{2 i}\left(\mathbb{F}_{q} ; \mathbb{Z} / \ell^{\infty}\right) \cong H^{0}\left(\mathbb{F}_{q} ; W(i)\right)=\mathbb{Z} / w_{i}\left(\mathbb{F}_{q}\right),
$$

while $K_{n}\left(\mathbb{F}_{q} ; \mathbb{Z} / \ell^{\infty}\right)=0$ if $n$ is odd or negative.

We now consider the $e$-invariant of a $p$-local field $E$ of characteristic zero. If $\mathcal{O}_{E}$ is its valuation ring we have $H^{0}\left(\mathcal{O}_{E} ; W(i)\right)=H^{0}(E ; W(i)) \cong \mathbb{Z} / w_{i}(E)$. Thus the following rigidity theorem for étale cohomology determines $w_{i}(E)$.

Proposition 1.11. Let $E$ be a p-local field of characteristic zero with valuation ring $\mathcal{O}_{E}$ and residue field $k_{\mathfrak{P}}$, and let $M$ be one of the coefficient modules $\mathbb{Z} / \ell^{\nu}(i)$, $W(i)$ or $\mathbb{Z}_{\ell}(i)$. Suppose $p \neq \ell$. Then the canonical map $\mathcal{O}_{E} \rightarrow k_{\mathfrak{P}}$ induces an isomorphism

$$
H^{n}\left(\mathcal{O}_{E} ; M\right) \stackrel{\cong}{\longrightarrow} H^{n}\left(k_{\mathfrak{P}} ; M\right)
$$

in every degree $n$. In particular $w_{i}(E)=w_{i}\left(k_{\mathfrak{P}}\right)$ for all $i$, and is finite for $i \neq 0$.

Proof. This is well known for $M=\mathbb{Z} / \ell^{\nu}(i)$; see $[\mathrm{M} 1$, III.3.11(a)] and/or $[\mathrm{CF}$, II.7(1)]. The other cases follow by passage to $\left(\mathrm{co}^{-}\right)$limits.

We may now determine the mod $2^{\infty} K$-groups of $p$-local fields of characteristic zero and totally imaginary number fields.

Theorem 1.12. Let $E$ be a p-local field of characteristic zero, and write $w_{i}$ for $w_{i}(E)$. The $\bmod 2^{\infty}$ algebraic $K$-groups of $E$ are:

$$
K_{n}\left(E ; \mathbb{Z} / 2^{\infty}\right) \cong \begin{cases}H^{0}(E ; W(i))=\mathbb{Z} / w_{i} & \text { for } n=2 i \geq 0 \\ H^{1}(E ; W(i)) & \text { for } n=2 i-1 \geq 1\end{cases}
$$

We can and shall make the group $K_{2 i-1}\left(E ; \mathbb{Z} / 2^{\infty}\right) \cong H^{1}(E ; W(i))$ explicit. When $p \neq 2$, it is the finite cyclic group $\mathbb{Z} / w_{i-1}$; see Corollary 2.10 and Theorem 3.4. When $p=2, i \geq 1$ and $d=\left[E: \mathbb{Q}_{2}\right]$ this group is $\left(\mathbb{Z} / 2^{\infty}\right)^{d} \oplus \mathbb{Z} / w_{i-1}$; see Proposition 3.6 and Theorem 3.7. 
Proof. Consider the mod $2^{\infty}$ Bloch-Lichtenbaum spectral sequence (1.3) for $E$, converging to $K_{*}\left(E ; \mathbb{Z} / 2^{\infty}\right)$. The $E_{2}$-term is

$$
E_{2}^{p, q}= \begin{cases}H^{p-q}(E ; W(-q)) & \text { for } q \leq p \leq 0 \\ 0 & \text { otherwise. }\end{cases}
$$

Now it is well known that $H^{n}(E ; W(i))=0$ for $n \geq 3$; this is part of the Local Duality Theorem [T, 2.1], which we have restated below in Theorem 2.11. By an elementary direct limit argument, detailed in Corollary 2.12, we also have $H^{2}(E ; W(i))=0$ when $i \neq 1$. Hence the spectral sequence is concentrated on the two diagonal lines where $0 \leq p-q \leq 1$, and the spectral sequence collapses at the $E_{2}$-term.

Theorem 1.13. Let $F$ be a totally imaginary number field. Its mod $2^{\infty}$ algebraic $K$-groups are given as follows:

$$
K_{n}\left(F ; \mathbb{Z} / 2^{\infty}\right) \cong \begin{cases}H^{0}(F ; W(i))=\mathbb{Z} / w_{i}(F) & \text { for } n=2 i \geq 0 \\ H^{1}(F ; W(i)) & \text { for } n=2 i-1 \geq 1 .\end{cases}
$$

Proof. The argument is similar to the proof of Theorem 1.12, except that the reference for $H^{2}(F ; W(i))=0$ when $i \geq 2$ is [W1, 7.3], restated below in Theorem $4.5(\mathrm{a})$.

Remark 1.14. There is a cyclic summand $E^{i}$ of $K_{2 i-1}(F)$ for $i>0$, constructed in [HS], called the Harris-Segal summand. If $F$ is totally imaginary, it was known that this summand has order $w_{i}(F)$, except possibly when $F$ is exceptional and $i \equiv 0 \bmod 4 . \quad\left(\right.$ See $[\mathrm{HS}]$ and $[\mathrm{W} 1,6.6 .2]$.) Since $K_{2 i}\left(F ; \mathbb{Z} / 2^{\infty}\right) \cong \mathbb{Z} / w_{i}(F)$ is an extension of $K_{2 i-1}(F)\{2\}$ by $K_{2 i}(F) \otimes \mathbb{Z} / 2^{\infty}$ we must have $E^{i} \cong \mathbb{Z} / w_{i}(F)$. Therefore this result resolves the ambiguity in the order of $E^{4 k}$, as well as showing that $E^{i}$ is all of the 2-torsion subgroup of $K_{2 i-1}(F)$.

Next we describe the mod $2 K$-theory of $p$-local fields of characteristic zero and totally imaginary number fields, in terms of étale cohomology groups. An alternative identification may be given using the well known facts that $H^{0}(F ; \mathbb{Z} / 2)=\mathbb{Z} / 2$, $H^{1}(F ; \mathbb{Z} / 2) \cong F^{\times} / 2$ and $H^{2}(F ; \mathbb{Z} / 2) \cong{ }_{2} \operatorname{Br}(F)$ for every field $F$. Moreover, if $E$ is a $p$-local field of characteristic zero, then $H^{2}(E ; \mathbb{Z} / 2) \cong \mathbb{Z} / 2$.

Recall that the notation $H^{2} \rtimes H^{0}$ represents an Abelian group extension of $H^{0}$ by $H^{2}$. For example, $\mathbb{Z} / 2 \rtimes \mathbb{Z} / 2$ represents either $\mathbb{Z} / 2 \oplus \mathbb{Z} / 2$ or $\mathbb{Z} / 4$.

Theorem 1.15. (a) Let $E$ be a p-local field of characteristic zero. Its mod 2 algebraic $K$-groups are given (up to extensions) as follows:

$$
K_{n}(E ; \mathbb{Z} / 2) \cong \begin{cases}H^{0}(E ; \mathbb{Z} / 2)=\mathbb{Z} / 2 & \text { for } n=0, \\ H^{1}(E ; \mathbb{Z} / 2) \cong E^{\times} / 2 & \text { for } n=2 i-1 \text { odd, } \\ H^{2}(E ; \mathbb{Z} / 2) \rtimes H^{0}(E ; \mathbb{Z} / 2) & \text { for } n=2 i>0 \text { even } .\end{cases}
$$

When $n=2 i>0$ the extension is either $\mathbb{Z} / 2 \oplus \mathbb{Z} / 2$ or $\mathbb{Z} / 4$; see Remark $1.16(a)$.

(b) Let $F$ be a totally imaginary number field. Its mod 2 algebraic $K$-groups are given (up to extensions) as follows:

$$
K_{n}(F ; \mathbb{Z} / 2) \cong \begin{cases}H^{0}(F ; \mathbb{Z} / 2)=\mathbb{Z} / 2 & \text { for } n=0, \\ H^{1}(F ; \mathbb{Z} / 2) \cong F^{\times} / 2 & \text { for } n=2 i-1 \text { odd }, \\ H^{2}(F ; \mathbb{Z} / 2) \rtimes H^{0}(F ; \mathbb{Z} / 2) & \text { for } n=2 i>0 \text { even. }\end{cases}
$$

When $n=2 i>0$ the extension may be nontrivial; see Remark $1.16(b)$. 
Proof. We give the proof in the number field case. The local case is identical.

When $F$ is totally imaginary we have $H^{n}(F ; \mathbb{Z} / 2)=0$ for $n \geq 3$; this is part of Tate's Theorem [T, 3.1(c)], which we have restated below as Theorem 4.2 (we refer the reader there for further discussion). The mod 2 Bloch-Lichtenbaum spectral sequence (1.2) for $F$ abuts to $K_{*}(F ; \mathbb{Z} / 2)$ and its nonzero $E_{2}$-terms $H^{p-q}(F ; \mathbb{Z} / 2)$ are concentrated on the three adjacent diagonal lines where $0 \leq p-q \leq 2$. Hence there is no room for nonzero differentials, the spectral sequence collapses at the $E_{2}$-term, and the $E_{\infty}$-term gives the asserted expression for $K_{*}(F ; \mathbb{Z} / 2)$.

Remarks 1.16. (a) The possibility of a nontrivial extension in $K_{2 i}(E ; \mathbb{Z} / 2)$ is related to the presence of nontrivial multiplications by $\eta$ in the 2-adic groups $K_{*}\left(E ; \mathbb{Z}_{2}\right)$. For example, we know that $K_{2}\left(\mathbb{Q}_{2} ; \mathbb{Z} / 2\right) \cong \mathbb{Z} / 4$ by an old calculation of Araki and Toda (see $[\mathrm{W} 1,1.3]$ ), since the symbol $\{-1,-1\}$ is nonzero in $K_{2}\left(\mathbb{Q}_{2}\right)$. More generally, $K_{4 j+2}\left(\mathbb{Q}_{2} ; \mathbb{Z} / 2\right) \cong \mathbb{Z} / 4$ for all $j \geq 0$ (see $[\mathrm{R}]$ or Example 3.8). In contrast, if $p \neq 2$ the extension in $K_{2 i}(E ; \mathbb{Z} / 2)$ is split for all $i>0$ (see [W1, 4.1(c)]).

(b) When $F$ is a totally imaginary number field, the extension in $K_{2 i}(F ; \mathbb{Z} / 2)$ may also be nontrivial, but in this case $H^{2}(F ; \mathbb{Z} / 2)={ }_{2} \operatorname{Br}(F)$ is infinitely generated. To illustrate, the field $F=\mathbb{Q}(\sqrt{-7})$ is contained in $\mathbb{Q}_{2}$, and the nontrivial extension in $K_{8 k+2}\left(\mathbb{Q}_{2} ; \mathbb{Z} / 2\right)$ detects a corresponding $\mathbb{Z} / 4$ in $K_{8 k+2}(\mathbb{Q}(\sqrt{-7}) ; \mathbb{Z} / 2)$ for all $k \geq$ 0 .

\section{Review of Étale COHOMOlogy}

Notation 2.1. We shall often use the Pontryagin dual $A^{\#}=\operatorname{Hom}(A, \mathbb{Q} / \mathbb{Z})$ of an Abelian group $A$. The correspondence $A \mapsto A^{\#}$ induces an equivalence between the category of discrete Abelian torsion groups (ind-finite groups) and the category of profinite Abelian groups, with $\left(A^{\#}\right)^{\#} \cong A$. (See e.g. [W2, Ex. 6.11.3].)

Lemma 2.2 (Exactness of $\lim$ ). If $\left\{0 \rightarrow A^{i} \rightarrow B^{i} \rightarrow C^{i} \rightarrow 0\right\}_{i \in I}$ is a filtered inverse system of exact sequences of profinite Abelian groups, then $0 \rightarrow \lim _{i} A^{i} \rightarrow$ $\lim _{i} B^{i} \rightarrow \lim _{i} C^{i} \rightarrow 0$ is exact, where $\lim _{i}$ denotes the inverse limit of the underlying system of Abelian groups.

Proof. By Pontryagin duality, the exactness of filtered colimits (direct limits) in the category of discrete Abelian torsion groups implies exactness of filtered limits (inverse limits) in the category of profinite Abelian groups. Hence it suffices to show that $\lim _{i}$ is the categorical limit. The forgetful functor from profinite Abelian groups to Abelian groups is right adjoint to the profinite completion functor. Any right adjoint preserves categorical limits. (See e.g. [W2, 2.6.10].) Hence the limit in the category of profinite Abelian groups coincides with the limit in the category of Abelian groups.

Notations 2.3. When $A$ is an Abelian group and $n$ an integer we write ${ }_{n} A$ and $A / n$ for the kernel and cokernel of the multiplication by $n$ map $n: A \rightarrow A$. We let tors $A \subseteq A$ denote the maximal torsion subgroup, and for a prime $\ell$ we let $A\{\ell\}=$ $\bigcup_{\nu} \ell^{\nu} A$ be the maximal $\ell$-torsion subgroup. We also let $A / \ell^{\infty}=\operatorname{colim}_{\nu} A / \ell^{\nu} \cong$ $A \otimes \mathbb{Z} / \ell^{\infty}$.

Let $\mathbb{G}_{m}(R)$ be the multiplicative group $R^{\times}$, let $\mu(R)$ be its torsion subgroup, i.e., the roots of unity in $R$, and let $\mu_{\ell^{\nu}}(R)$ be the $\ell^{\nu}$ th roots of unity in $R$, with $\mu_{\ell \infty}(R)$ denoting all $\ell$ th power roots of unity in $R$. 
We may and shall regard the groups $\mu_{\ell^{\nu}}$ as sheaves for the étale topology. For $i \geq 0$ let $\mu_{\ell^{\nu}}^{\otimes i}$ be the tensor product of $i$ copies of $\mu_{\ell^{\nu}}$, and let $\mu_{\ell^{\nu}}^{\otimes i}=\left(\mu_{\ell^{\nu}}^{\otimes(-i)}\right)^{\#}$ (Pontryagin dual) for $i<0$. We will prefer additive notation for these coefficient sheaves, writing $\mathbb{Z} / \ell^{\nu}(i)$ for $\mu_{\ell^{\nu}}^{\otimes i}$. We let $\mathbb{Z}_{\ell}(i)=\lim _{\nu} \mathbb{Z} / \ell^{\nu}(i)$ be the limit over the coefficient projections $\pi: \mathbb{Z} / \ell^{\nu+1}(i) \rightarrow \mathbb{Z} / \ell^{\nu}(i)$. Dually we let $W(i)=\operatorname{colim}_{\nu} \mathbb{Z} / \ell^{\nu}(i)$ be the colimit over the coefficient injections $\iota: \mathbb{Z} / \ell^{\nu}(i) \rightarrow \mathbb{Z} / \ell^{\nu+1}(i)$. Then $\mathbb{Z}_{\ell}(i)$ is a profinite group and $W(i)$ is a discrete torsion group for all $i$. (The sheaves $\mu_{\ell^{\nu}}^{\otimes i}$ are discussed in [M1, p. 163], along with the $\ell$-adic sheaves $\mathbb{Z}_{\ell}(i)$.)

The definition of the étale cohomology groups $H^{n}(R ; M)$ can be naturally extended to the topologized coefficient sheaf $\mathbb{Z}_{\ell}(i)$; see $[\mathrm{J}]$. There is then an exact sequence

$$
0 \rightarrow \lim _{\nu}^{1} H^{n-1}\left(R ; \mathbb{Z} / \ell^{\nu}(i)\right) \rightarrow H^{n}\left(R ; \mathbb{Z}_{\ell}(i)\right) \rightarrow \lim _{\nu} H^{n}\left(R ; \mathbb{Z} / \ell^{\nu}(i)\right) \rightarrow 0 .
$$

The $\lim ^{1}$-term often vanishes, e.g. when each group $H^{n-1}\left(R ; \mathbb{Z} / \ell^{\nu}(i)\right)$ is finite. There is always an isomorphism

$$
H^{n}(R ; W(i)) \cong \operatorname{colim}_{\nu} H^{n}\left(R ; \mathbb{Z} / \ell^{\nu}(i)\right)
$$

(See [M1, III.3.6(d)].)

The Picard group $\operatorname{Pic}(R)$ and Brauer group $\operatorname{Br}(R)$ of a ring $R$ are classically defined in terms of constant rank one projective $R$-modules and Azumaya $R$-algebras, respectively. It is well known that $\operatorname{Pic}(R) \cong H^{1}\left(R ; \mathbb{G}_{m}\right)$, while $\operatorname{Br}(R) \cong H^{2}\left(R ; \mathbb{G}_{m}\right)$ by a theorem of Gabber and Hoobler. (See [M1, Ch. IV] and [H].) When $F$ is a field, $\operatorname{Pic}(F)=H^{1}\left(F ; \mathbb{G}_{m}\right)=0$ by Hilbert's Theorem 90 , and the Brauer group is the classical group of Morita equivalence classes of central simple $F$-algebras.

We refer to the following result as the universal coefficient theorem.

Proposition 2.4. (a) Suppose $H^{n}\left(R ; \mathbb{Z}_{\ell}(i)\right)$ is a finitely generated $\mathbb{Z}_{\ell}$-module, for a fixed $i$ and all $n$. We can then write

$$
H^{n}\left(R ; \mathbb{Z}_{\ell}(i)\right) \cong F^{n}(i) \oplus T^{n}(i)
$$

where $F^{n}(i)$ is a finitely generated free $\mathbb{Z}_{\ell}$-module and $T^{n}(i)=\operatorname{tors} H^{n}\left(R ; \mathbb{Z}_{\ell}(i)\right)$ is a finite $\ell$-group. Then

$$
H^{n}(R ; W(i)) \cong F^{n}(i)^{\#} \oplus T^{n+1}(i) .
$$

(b) Conversely suppose that $H^{n}(R ; W(i))$ is the Pontryagin dual of a finitely generated $\mathbb{Z}_{\ell}$-module, for a fixed $i$ and all $n$. We can then write

$$
H^{n}(R ; W(i)) \cong F^{n}(i)^{\#} \oplus U^{n}(i)^{\#}
$$

where $F^{n}(i)$ is a finitely generated free $\mathbb{Z}_{\ell}$-module and $U^{n}(i)=\operatorname{tors}\left(H^{n}(R ; W(i))^{\#}\right)$ is a finite l-group. Then

$$
H^{n}\left(R ; \mathbb{Z}_{\ell}(i)\right) \cong F^{n}(i) \oplus U^{n-1}(i)^{\#} .
$$

Proof. (a) The short exact sequence of coefficient sheaves $0 \rightarrow \mathbb{Z}_{\ell}(i) \stackrel{\ell^{\nu}}{\rightarrow} \mathbb{Z}_{\ell}(i) \rightarrow$ $\mathbb{Z} / \ell^{\nu}(i) \rightarrow 0$ induces a long exact sequence in étale cohomology, which leads to the following short exact sequence:

$$
0 \rightarrow H^{n}\left(R ; \mathbb{Z}_{\ell}(i)\right) / \ell^{\nu} \rightarrow H^{n}\left(R ; \mathbb{Z} / \ell^{\nu}(i)\right) \rightarrow \ell^{\nu} H^{n+1}\left(R ; \mathbb{Z}_{\ell}(i)\right) \rightarrow 0 .
$$


Inserting the formulas for $H^{n}\left(R ; \mathbb{Z}_{\ell}(i)\right)$ and passing to the colimit over $\nu$ then gives the short exact sequence

$$
0 \rightarrow F^{n}(i) / \ell^{\infty} \rightarrow H^{n}(R ; W(i)) \rightarrow T^{n+1}(i) \rightarrow 0,
$$

which is split since $F^{n}(i) / \ell^{\infty} \cong F^{n}(i)^{\#}$ is injective.

(b) The short exact sequence of coefficient sheaves $0 \rightarrow \mathbb{Z} / \ell^{\nu}(i) \rightarrow W(i) \stackrel{\ell^{\nu}}{\rightarrow}$ $W(i) \rightarrow 0$ induces a long exact sequence in étale cohomology, which provides the following short exact sequence:

$$
0 \rightarrow H^{n-1}(R ; W(i)) / \ell^{\nu} \rightarrow H^{n}\left(R ; \mathbb{Z} / \ell^{\nu}(i)\right) \rightarrow \ell^{\nu} H^{n}(R ; W(i)) \rightarrow 0 .
$$

The finite generation hypothesis on $H^{n}(R ; W(i))^{\#}$ ensures that each term in this exact sequence is a finite group. Inserting the formulas for $H^{n}(R ; W(i))$ and passing to the limit over $\nu$ gives the short exact sequence

$$
0 \rightarrow U^{n-1}(i)^{\#} \rightarrow H^{n}\left(R ; \mathbb{Z}_{\ell}(i)\right) \rightarrow F^{n}(i) \rightarrow 0,
$$

which is split since $F^{n}(i)$ is projective. The $\lim ^{1}$-terms vanish, since the groups in the limit system are finite.

Notations 2.5. Let $E$ be a $p$-local field of characteristic zero, i.e., a finite extension of the $p$-adic numbers $\mathbb{Q}_{p}$. Let $e$ be its ramification index and $f$ its residue field degree. The degree of $E$ over $\mathbb{Q}_{p}$ is $\left[E: \mathbb{Q}_{p}\right]=e f$. Let $\mathcal{O}_{E} \subset E$ be the valuation ring, let $\mathfrak{P} \subset \mathcal{O}_{E}$ be its maximal ideal, and let $k_{\mathfrak{P}}=\mathcal{O}_{E} / \mathfrak{P}$ be the residue field.

Let $F$ be a number field, i.e., a finite extension of the rational numbers $\mathbb{Q}$, with $r_{1}$ real embeddings and $r_{2}$ pairs of complex embeddings. The degree of $F$ over $\mathbb{Q}$ is $[F: \mathbb{Q}]=r_{1}+2 r_{2}$. Let $\mathcal{O}_{F} \subset F$ be the ring of algebraic integers, and let $R=\mathcal{O}_{F}\left[\frac{1}{\ell}\right]$ be the ring of $\ell$-integers in $F$. As in the local case, let $k_{\mathfrak{P}}=\mathcal{O}_{F} / \mathfrak{P}$ be the residue field at $\mathfrak{P}$.

Let $S=\{\mathfrak{P} \mid \ell\}$ be the set of prime ideals (viewed as primes, or places) in $\mathcal{O}_{F}$ dividing $\ell$, and let $s=\# S$ be the number of such primes. Let $S_{\infty}$ be the union of $S$ and the set of Archimedean places of $F$, and let $\Sigma$ be the set of all places of $F$. For each place $v$ of $F$ let $F_{v}$ denote the $v$-completion of $F$. For each fixed prime $p$, we have an identity

$$
\sum_{\mathfrak{P} \mid p}\left[F_{\mathfrak{P}}: \mathbb{Q}_{p}\right]=[F: \mathbb{Q}]
$$

where the sum runs over the primes $\mathfrak{P}$ dividing $p$. (See e.g. [CF, II.2(4)].)

For a $p$-local field $E$ of characteristic zero there is an isomorphism $\operatorname{Br}(E) \cong \mathbb{Q} / \mathbb{Z}$, while in the Archimedean cases $\operatorname{Br}(\mathbb{R}) \cong \mathbb{Z} / 2$ and $\operatorname{Br}(\mathbb{C})=0$. (See e.g. pp. $162-163$ of $[\mathrm{Se}]$.

The Brauer group of a number field $F$ is determined by the Brauer-HasseNoether theorem, asserting exactness of the sequence

$$
0 \rightarrow \operatorname{Br}(F) \stackrel{\beta}{\rightarrow} \bigoplus_{v \in \Sigma} \operatorname{Br}\left(F_{v}\right) \stackrel{h}{\rightarrow} \mathbb{Q} / \mathbb{Z} \rightarrow 0,
$$

where $h$ is a sum of injections $\operatorname{Br}\left(F_{v}\right) \rightarrow \mathbb{Q} / \mathbb{Z}$. (See sections 10 and 11 of Chapter VII in $[\mathrm{CF}]$.) There is a similar isomorphism $\operatorname{Br}(R) \cong(\mathbb{Z} / 2)^{r_{1}} \oplus(\mathbb{Q} / \mathbb{Z})^{s-1}$ and an exact sequence

$$
0 \rightarrow \operatorname{Br}(R) \stackrel{\beta}{\rightarrow} \bigoplus_{v \in S_{\infty}} \operatorname{Br}\left(F_{v}\right) \stackrel{h}{\rightarrow} \mathbb{Q} / \mathbb{Z} \rightarrow 0 .
$$


(See [M1, p. 109]; $S_{\infty}$ contains non-Archimedean primes, so the map $h$ is surjective and we may truncate the sequence in loc. cit. after the first three terms.) Also note that the natural map $\operatorname{Br}(R) \rightarrow \operatorname{Br}(F)$ is an injection. (See [M1, p. 107].)

Since $\mathbb{Z} / \ell^{\nu}(1)=\mu_{\ell^{\nu}}$, the Kummer sequence allows us to describe the étale cohomology groups with twist 1 in terms of units, the Picard group and the Brauer group. For any ring $R$ we have $H^{0}\left(R ; \mathbb{Z} / \ell^{\nu}(1)\right) \cong \mu_{\ell^{\nu}}(R), H^{1}\left(R ; \mathbb{Z} / \ell^{\nu}(1)\right) \cong$ $R^{\times} / \ell^{\nu} \rtimes \ell^{\nu} \operatorname{Pic}(R)$ and $H^{2}\left(R ; \mathbb{Z} / \ell^{\nu}(1)\right) \cong \operatorname{Pic}(R) / \ell^{\nu} \rtimes \ell^{\nu} \operatorname{Br}(R)$.

Proposition 2.7. (a) Let $E$ be a p-local field of characteristic zero. Then

$$
\begin{aligned}
& H^{0}(E ; W(1)) \cong \mu_{\ell^{\infty}}(E), \\
& H^{1}(E ; W(1)) \cong \begin{cases}\mathbb{Z} / \ell^{\infty} & \text { for } p \neq \ell, \\
\left(\mathbb{Z} / \ell^{\infty}\right)^{e f+1} & \text { for } p=\ell,\end{cases} \\
& H^{2}(E ; W(1)) \cong \operatorname{Br}(E)\{\ell\} \cong \mathbb{Z} / \ell^{\infty} .
\end{aligned}
$$

(b) Let $R$ be the ring of $\ell$-integers in a number field. Then $\operatorname{Pic}(R)$ is a finite group, and

$$
\begin{aligned}
& H^{0}(R ; W(1)) \cong \mu_{\ell \infty}(R), \\
& H^{1}(R ; W(1)) \cong\left(\mathbb{Z} / \ell^{\infty}\right)^{r_{1}+r_{2}+s-1} \oplus \operatorname{Pic}(R)\{\ell\}, \\
& H^{2}(R ; W(1)) \cong \operatorname{Br}(R)\{\ell\} \cong(\mathbb{Z} /(2, \ell))^{r_{1}} \oplus\left(\mathbb{Z} / \ell^{\infty}\right)^{s-1} .
\end{aligned}
$$

Proof. By the Dirichlet unit theorem $R^{\times} \cong \mu(R) \times \mathbb{Z}^{r_{1}+r_{2}+s-1}$, while $E^{\times} \cong \mathbb{Z} \times$ $\mu(E) \times \mathbb{Z}_{p}^{e f}$. (See e.g. [N, III.1.2].) Passing to colimits over $\nu$ gives the result.

Example 2.8. When $\ell=2$ and $R=\mathbb{Z}\left[\frac{1}{2}\right]$ both $\operatorname{Br}(R) \cong \mathbb{Z} / 2$ and $\operatorname{Br}(\mathbb{R}) \cong \mathbb{Z} / 2$. The natural map therefore induces an isomorphism $H^{2}\left(\mathbb{Z}\left[\frac{1}{2}\right] ; \mathbb{Z} / 2\right) \cong H^{2}(\mathbb{R} ; \mathbb{Z} / 2) \cong$ $\mathbb{Z} / 2$. We will need this special case in section 5 below.

There is a long exact localization sequence in étale cohomology, defined and natural for Dedekind domains. We shall need the following two cases.

Proposition 2.9. (a) Let $E$ be a p-local field of characteristic zero with valuation ring $\mathcal{O}_{E}$ and residue field $k_{\mathfrak{P}}$. The localization sequence breaks up into short exact sequences

$$
0 \rightarrow H^{n}\left(\mathcal{O}_{E} ; W(i)\right) \rightarrow H^{n}(E ; W(i)) \stackrel{\partial_{\mathfrak{P}}^{e}}{\longrightarrow} H^{n-1}\left(k_{\mathfrak{P}} ; W(i-1)\right) \rightarrow 0
$$

for all integers $i$. Hence $H^{n}\left(\mathcal{O}_{E} ; W(i)\right) \cong H^{n}(E ; W(i))$ when $n \neq 1,2$, or when $n=2$ and $i \neq 1$.

(b) Let $F$ be a number field with ring of $\ell$-integers $R$ and residue fields $k_{\mathfrak{P}}$ for $\mathfrak{P} \nmid \ell$. The localization sequence breaks up into exact sequences

$$
\begin{aligned}
0 \rightarrow H^{1}(R ; W(i)) & \rightarrow H^{1}(F ; W(i)) \stackrel{\partial^{e}}{\longrightarrow} \bigoplus_{\mathfrak{P} \nmid \ell} H^{0}\left(k_{\mathfrak{P}} ; W(i-1)\right) \rightarrow \\
& \rightarrow H^{2}(R ; W(i)) \rightarrow H^{2}(F ; W(i)) \stackrel{\partial^{e}}{\longrightarrow} \bigoplus_{\mathfrak{P} \nmid \ell} H^{1}\left(k_{\mathfrak{P}} ; W(i-1)\right)
\end{aligned}
$$

for all integers $i$. When $i \neq 1$ the rightmost group is zero. For $n \neq 1,2$ there are isomorphisms

$$
H^{n}(R ; W(i)) \stackrel{\cong}{\longrightarrow} H^{n}(F ; W(i)) .
$$


We shall see in Proposition 4.7 below that $H^{2}(R ; W(i)) \rightarrow H^{2}(F ; W(i))$ is an isomorphism for $i \geq 2$, and an injection when $i=1$. Hence the localization sequence in (b) breaks up into short exact sequences for $i \geq 1$.

Proof. The localization sequences with coefficients $\mathbb{Z} / \ell^{\nu}(i)$ are given in [So, III.1] for $\ell$ odd, and as [W1, 4.0] for $\ell=2$. (The hypothesis in loc. cit. that $i \geq 1$ is not needed. See [So, p. 268].) The proposition follows by passing to the colimit over $\nu$, and noting that $H^{n}\left(k_{\mathfrak{P}} ; W(i-1)\right)=0$ for $n \neq 0$, except for $(n, i)=(1,1)$, by Proposition 1.10.

Corollary 2.10. Let $E$ be a p-local field of characteristic zero with residue field $k_{\mathfrak{P}}$ and $p \neq \ell$. For $i \neq 0,1$ we have

$$
H^{n}(E ; W(i)) \cong \begin{cases}\mathbb{Z} / w_{i}\left(k_{\mathfrak{P}}\right) & \text { for } n=0 \\ \mathbb{Z} / w_{i-1}\left(k_{\mathfrak{P}}\right) & \text { for } n=1, \\ 0 & \text { otherwise. }\end{cases}
$$

Moreover, the connecting map $\partial_{\mathfrak{P}}^{e}: H^{1}(E ; W(i)) \rightarrow H^{0}\left(k_{\mathfrak{P}} ; W(i-1)\right)$ in the étale cohomology localization sequence $2.9(a)$ is an isomorphism for all $i \neq 0$.

Proof. This follows by combining Propositions 1.8, 1.10, 1.11 and 2.9(a).

Next we review local Tate-Poitou duality. Let $E$ be a $p$-local field of characteristic zero, and let $M$ be a finite $G_{E}$-module. We define the (twisted) dual module to be $M^{\prime}=\operatorname{Hom}\left(M, \mathbb{G}_{m}\right)$. The example $\mathbb{Z} / \ell^{\nu}(i)^{\prime} \cong \mathbb{Z} / \ell^{\nu}(1-i)$ will be important to us. The pairing $M \times M^{\prime} \rightarrow \mathbb{G}_{m}$ induces a pairing

$$
H^{n}(E ; M) \times H^{2-n}\left(E ; M^{\prime}\right) \rightarrow H^{2}\left(E ; \mathbb{G}_{m}\right)=\operatorname{Br}(E) \cong \mathbb{Q} / \mathbb{Z} .
$$

The following result appears as [T, 2.1], or [M2, I.2.3].

Local Duality Theorem 2.11 (Tate, Poitou). Let E be a p-local field of characteristic zero and $M$ a finite coefficient module. Then for all $n$ the pairing defined above is a perfect pairing of finite groups

$$
H^{n}(E ; M) \times H^{2-n}\left(E ; M^{\prime}\right) \rightarrow \mathbb{Q} / \mathbb{Z},
$$

natural in $E$ and $M$. In particular, $H^{n}(E ; M)=0$ for all $n \neq 0,1,2$.

Corollary 2.12. Let $E$ be a p-local field of characteristic zero. Each $H^{n}(E ; W(i))$ is a discrete torsion group, each $H^{n}\left(E ; \mathbb{Z}_{\ell}(i)\right)$ is a profinite group, and there is a perfect pairing

$$
H^{n}(E ; W(i)) \times H^{2-n}\left(E ; \mathbb{Z}_{\ell}(1-i)\right) \rightarrow \mathbb{Q} / \mathbb{Z}
$$

for all $n$ and $i$.

In particular $H^{n}(E ; W(i))=0$ and $H^{n}\left(E ; \mathbb{Z}_{\ell}(i)\right)=0$ for all $n \neq 0,1,2$.

Finally $H^{2}(E ; W(1)) \cong \mathbb{Z} / \ell^{\infty}$ but $H^{2}(E ; W(i))=0$ for all $i \neq 1$.

Proof. Tate-Poitou duality gives isomorphisms of finite groups $H^{n}\left(E ; \mathbb{Z} / \ell^{\nu}(i)\right) \rightarrow$ $H^{2-n}\left(E ; \mathbb{Z} / \ell^{\nu}(1-i)\right)^{\#}$ for all $\nu$. We claim that these are compatible with the transition maps $\iota_{*}$ in the direct system defining $H^{n}(E ; W(i))$, and the Pontryagin duals $\pi_{*}^{\#}$ of the transition maps in the inverse system defining $H^{2-n}\left(E ; \mathbb{Z}_{\ell}(1-i)\right)$. 
To see this, consider the maps induced by the following commutative diagram of coefficient modules:

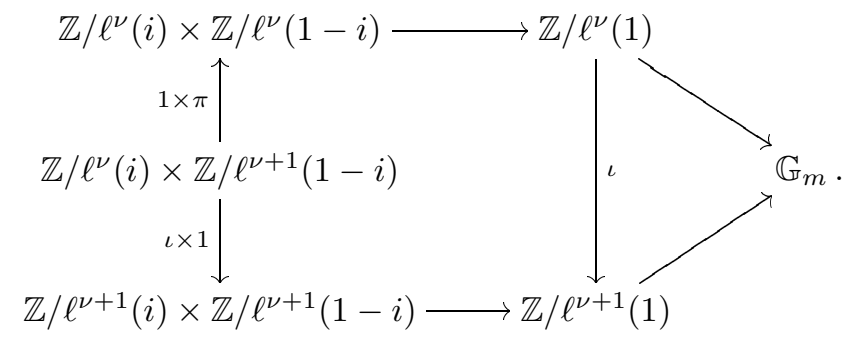

Passing to the colimit over $\nu$, and recalling that Pontryagin duality converts colimits of discrete torsion groups to limits of profinite groups, we obtain a duality isomorphism $H^{n}(E ; W(i)) \rightarrow H^{2-n}\left(E ; \mathbb{Z}_{\ell}(1-i)\right)^{\#}$, as claimed.

For the final claim, assume that $i \neq 1$. Then $w_{i-1}(E)$ is finite by Proposition 1.11. Now

$$
H^{2}\left(E ; \mathbb{Z} / \ell^{\nu}(i)\right) \cong H^{0}\left(E ; \mathbb{Z} / \ell^{\nu}(1-i)\right)^{\#} \cong \mathbb{Z} / w_{i-1}(E)
$$

for large $\nu$, and the maps in the inverse system are multiplication by $\ell$. Hence the dual maps in the direct system are nilpotent, and the colimit $H^{2}(E ; W(i))$ is trivial.

Proposition 2.13. Let $E$ be a p-local field of characteristic zero. Then

$$
\begin{aligned}
& H^{0}(E ; W(0)) \cong \mathbb{Z} / \ell^{\infty}, \\
& H^{1}(E ; W(0)) \cong \begin{cases}\mathbb{Z} / \ell^{\infty} \oplus \mu_{\ell^{\infty}}(E) & \text { for } p \neq \ell, \\
\left(\mathbb{Z} / \ell^{\infty}\right)^{e f+1} \oplus \mu_{\ell^{\infty}}(E) & \text { for } p=\ell,\end{cases} \\
& H^{2}(E ; W(0))=0 .
\end{aligned}
$$

Proof. This is a direct corollary of the universal coefficient theorem 2.4, Proposition 2.7 and local Tate-Poitou duality 2.12.

Remark 2.14. Combining Proposition 2.7, Corollary 2.10 and Proposition 2.13 we have shown that when $E$ is a $p$-local field of characteristic zero with $p \neq \ell$, then for all $n$ and $i$ the Abelian group $H^{n}(E ; W(i))$ is a discrete torsion group, Pontryagin dual to a finitely generated $\mathbb{Z}_{\ell}$-module. In Proposition 3.6 below we will obtain a similar result for $p=\ell=2$.

\section{Two-Primary algebraic $K$-Theory of two-LOCAL FIELDS}

In this section we compute the 2-completed algebraic $K$-theory of a $p$-local field $E$ of characteristic zero, $K_{n}\left(E ; \mathbb{Z}_{2}\right)$. When $p \neq 2$, this follows from the localization sequence in $K$-theory and Gabber's Rigidity Theorem; we give a slightly more general result in Theorem 3.4 below. For $p=2$ we need a result of Wagoner, the Bloch-Lichtenbaum spectral sequence, and local Tate-Poitou duality. The result is given in Theorem 3.7, thus proving Theorem 0.5 of the introduction.

There is a $K$-theory localization sequence for Dedekind domains, constructed in [Q2]. For $p$-local fields of characteristic zero it specializes as follows. 
Theorem 3.1 (Soulé). Let $E$ be a p-local field of characteristic zero with valuation ring $\mathcal{O}_{E}$ and residue field $k_{\mathfrak{P}}$. For each prime $\ell \neq p$, the $K$-theory localization sequence breaks up into split short exact sequences

$$
0 \rightarrow K_{2 i-1}\left(\mathcal{O}_{E} ; \mathbb{Z} / \ell^{\infty}\right) \rightarrow K_{2 i-1}\left(E ; \mathbb{Z} / \ell^{\infty}\right) \stackrel{\partial_{\mathfrak{P}}}{\longrightarrow} K_{2 i-2}\left(k_{\mathfrak{P}} ; \mathbb{Z} / \ell^{\infty}\right) \rightarrow 0,
$$

and isomorphisms $K_{2 i}\left(\mathcal{O}_{E} ; \mathbb{Z} / \ell^{\infty}\right) \cong K_{2 i}\left(E ; \mathbb{Z} / \ell^{\infty}\right)$.

Proof. The corresponding sequences with coefficients in $\mathbb{Z} / \ell^{\nu}$ are given in [So, III.3] for $\ell$ odd, and as (4.0) and Proposition 4.1(c) of [W1] for $\ell=2$. Now pass to the colimit over $\nu$ and note that $K_{n}\left(k_{\mathfrak{P}} ; \mathbb{Z} / \ell^{\infty}\right)=0$ for $n$ odd by Proposition 1.10. This yields the asserted exact sequences and isomorphisms.

Furthermore, each short exact sequence with $\mathbb{Z} / \ell^{\nu}$-coefficients is split, by the same references, so since $K_{2 i-2}\left(k_{\mathfrak{P}} ; \mathbb{Z} / \ell^{\nu}\right) \rightarrow K_{2 i-2}\left(k_{\mathfrak{P}} ; \mathbb{Z} / \ell^{\infty}\right)$ is an isomorphism for large $\nu$ it follows that also the exact sequence with $\mathbb{Z} / \ell^{\infty}$-coefficients is split.

Rigidity Theorem 3.2 (Gabber). Let $E$ be a p-local field of characteristic zero with $p \neq \ell$. The canonical map $\mathcal{O}_{E} \rightarrow k_{\mathfrak{P}}$ induces an isomorphism for all $n$ :

$$
K_{n}\left(\mathcal{O}_{E} ; \mathbb{Z} / \ell^{\infty}\right) \stackrel{\cong}{\longrightarrow} K_{n}\left(k_{\mathfrak{P}} ; \mathbb{Z} / \ell^{\infty}\right) .
$$

Proof. The corresponding result with $\mathbb{Z} / \ell^{\nu}$-coefficients is Theorem 1 of [Ga]. The result then follows by passing to the colimit.

Corollary 3.3. Let $E$ be a p-local field of characteristic zero with $p \neq \ell$. Then the connecting map

$$
\partial_{\mathfrak{P}}: K_{2 i-1}\left(E ; \mathbb{Z} / \ell^{\infty}\right) \rightarrow K_{2 i-2}\left(k_{\mathfrak{P}} ; \mathbb{Z} / \ell^{\infty}\right)
$$

in the $K$-theory localization sequence is an isomorphism for all $i$.

Proof. This follows because $K_{2 i-1}\left(\mathcal{O}_{E} ; \mathbb{Z} / \ell^{\infty}\right) \cong K_{2 i-1}\left(k_{\mathfrak{P}} ; \mathbb{Z} / \ell^{\infty}\right)=0$.

Thus the $\ell$-completed algebraic $K$-theory of $p$-local fields of characteristic zero is entirely known for $p \neq \ell$.

Theorem 3.4. Let $E$ be a $p$-local field of characteristic zero with $p \neq \ell$, and set $w_{i}=w_{i}(E)=w_{i}\left(k_{\mathfrak{P}}\right)$. Then

$$
K_{n}\left(E ; \mathbb{Z} / \ell^{\infty}\right) \cong \begin{cases}K_{n}\left(k_{\mathfrak{P}} ; \mathbb{Z} / \ell^{\infty}\right) \cong \mathbb{Z} / w_{i} & \text { when } n=2 i \text { is even }, \\ K_{n-1}\left(k_{\mathfrak{P}} ; \mathbb{Z} / \ell^{\infty}\right) \cong \mathbb{Z} / w_{i} & \text { when } n=2 i+1 \text { is odd }\end{cases}
$$

Hence

$$
K_{n}\left(E ; \mathbb{Z}_{\ell}\right) \cong \begin{cases}\mathbb{Z}_{\ell} & \text { for } n=0 \\ \mathbb{Z}_{\ell} \oplus \mathbb{Z} / w_{1} & \text { for } n=1 \\ K_{n-1}\left(k_{\mathfrak{P}} ; \mathbb{Z}_{\ell}\right) \cong \mathbb{Z} / w_{i} & \text { for } n=2 i>0 \text { even } \\ K_{n}\left(k_{\mathfrak{P}} ; \mathbb{Z}_{\ell}\right) \cong \mathbb{Z} / w_{i} & \text { for } n=2 i-1>1 \text { odd }\end{cases}
$$

Proof. By Proposition 1.10 the groups $K_{*}\left(k_{\mathfrak{P}} ; \mathbb{Z} / \ell^{\infty}\right)$ are concentrated in even degrees. Hence the localization sequence in Theorem 3.1 breaks up into isomorphisms and zero maps. This gives the $\bmod \ell^{\infty}$ calculation, and the other claim follows by the universal coefficient theorem.

Now suppose $p=\ell$. The $p$-completed algebraic $K$-groups of $p$-local fields of characteristic zero are known modulo torsion. 
Theorem 3.5 (Wagoner). Let $E$ be a p-local field of degree ef over $\mathbb{Q}_{p}$. For each $n$ the group $K_{n}\left(E ; \mathbb{Z}_{p}\right)$ is a finitely generated $\mathbb{Z}_{p}$-module, with

$$
\operatorname{dim}_{\mathbb{Q}_{p}}\left(K_{n}\left(E ; \mathbb{Z}_{p}\right) \otimes_{\mathbb{Z}_{p}} \mathbb{Q}_{p}\right)= \begin{cases}1 & \text { for } n=0, \\ \text { ef }+1 & \text { for } n=1, \\ 0 & \text { for } n>0 \text { even }, \\ \text { ef } & \text { for } n>1 \text { odd } .\end{cases}
$$

Thus by the universal coefficient theorem

$$
K_{n}\left(E ; \mathbb{Z} / p^{\infty}\right) \cong \begin{cases}\mathbb{Z} / p^{\infty} & \text { for } n=0 \\ \left(\mathbb{Z} / p^{\infty}\right)^{e f+1} & \text { for } n=1, \\ \text { tors } K_{n-1}\left(E ; \mathbb{Z}_{p}\right) & \text { for } n>0 \text { even } \\ \left(\mathbb{Z} / p^{\infty}\right)^{\text {ef }} \oplus \text { tors } K_{n-1}\left(E ; \mathbb{Z}_{p}\right) & \text { for } n>1 \text { odd } .\end{cases}
$$

For a proof, see $[\mathrm{Wg}]$. See $[\mathrm{P}]$ for the identification of the completed algebraic $K$-groups above with the continuous algebraic $K$-groups considered by Wagoner. Note that the case $n=1$ follows easily from the Dirichlet unit theorem.

Now assume $E$ is a 2-local field of characteristic zero, i.e., $p=\ell=2$. The following result uses Wagoner's result and our Theorem 1.12 to compute étale cohomology groups. In return it completes the explicit description of the groups $K_{n}\left(E ; \mathbb{Z} / 2^{\infty}\right)$ begun in Theorem 1.12.

Proposition 3.6. If $E$ is a 2-local field of characteristic zero, then the group $H^{n}(E ; W(i))$ is the Pontryagin dual of a finitely generated $\mathbb{Z}_{2}$-module for all $n$ and $i$. For $i \neq 0,1$ we have

$$
H^{n}(E ; W(i)) \cong \begin{cases}\mathbb{Z} / w_{i}(E) & \text { for } n=0 \\ \left(\mathbb{Z} / 2^{\infty}\right)^{\text {ef }} \oplus \mathbb{Z} / w_{i-1}(E) & \text { for } n=1, \\ 0 & \text { otherwise. }\end{cases}
$$

See Propositions 2.7 and 2.13 regarding $H^{n}(E ; W(1))$ and $H^{n}(E ; W(0))$.

Proof. Recall from Proposition 2.12 that we have $H^{n}(E ; W(i))=0$ for all $n \geq 3$, and for $n=2$ if $i \neq 1$. Also, if $i \neq 0$, then $H^{0}(E ; W(i))=\mathbb{Z} / w_{i}(E)$ is finite by Proposition 1.11. This leaves only $n=1$ to consider.

First assume $i>1$. Combining Wagoner's result (Theorem 3.5) for $p=2$ with Theorem 1.12 we find that $H^{1}(E ; W(i)) \cong\left(\mathbb{Z} / 2^{\infty}\right)^{e f} \oplus$ tors $K_{2 i-2}\left(E ; \mathbb{Z}_{2}\right)$, where tors $K_{2 i-2}\left(E ; \mathbb{Z}_{2}\right)$ is a finite group.

Hence $H^{n}(E ; W(i))$ is the Pontryagin dual of a finitely generated $\mathbb{Z}_{2}$-module for all $n$ and for $i>1$. Thus by the universal coefficient theorem $H^{n}\left(E ; \mathbb{Z}_{2}(i)\right)$ is a finitely generated $\mathbb{Z}_{2}$-module for all $n$ and for $i>1$. By local Tate-Poitou duality 2.12 the corresponding assertions hold for $H^{n}(E ; W(1-i))$ and $H^{n}\left(E ; \mathbb{Z}_{2}(1-i)\right)$. Combined with Propositions 2.4, 2.7 and 2.13 this proves finite generation of $H^{n}(E ; W(i))^{\#}$ and $H^{n}\left(E ; \mathbb{Z}_{2}(i)\right)$ for all integers $n$ and $i$.

In more detail, we can compute with the universal coefficient theorem and obtain the formulas $H^{0}\left(E ; \mathbb{Z}_{2}(i)\right)=0, H^{1}\left(E ; \mathbb{Z}_{2}(i)\right) \cong \mathbb{Z}_{2}^{e f} \oplus \mathbb{Z} / w_{i}(E)$ and $H^{2}\left(E ; \mathbb{Z}_{2}(i)\right) \cong$ tors $K_{2 i-2}\left(E ; \mathbb{Z}_{2}\right)$ for $i>1$. Then by local Tate-Poitou duality 2.12 we have tors $K_{2 i-2}\left(E ; \mathbb{Z}_{2}\right) \cong H^{2}\left(E ; \mathbb{Z}_{2}(i)\right) \cong H^{0}(E ; W(1-i))^{\#} \cong \mathbb{Z} / w_{i-1}(E)$, which proves the asserted formulas. 
We can now compute the 2-completed algebraic $K$-theory of any 2-local field of characteristic zero, and of its corresponding ring of integers.

Theorem 3.7. Let $E$ be a 2-local field of degree ef over $\mathbb{Q}_{2}$, with valuation ring $\mathcal{O}_{E}$. The 2-adically completed algebraic $K$-groups of $E$ and $\mathcal{O}_{E}$ are

$$
K_{n}\left(E ; \mathbb{Z}_{2}\right) \cong \begin{cases}\mathbb{Z}_{2} & \text { for } n=0, \\ \mathbb{Z}_{2}^{e f+1} \oplus \mathbb{Z} / w_{1}(E) & \text { for } n=1, \\ \mathbb{Z} / w_{i}(E) & \text { for } n=2 i>0 \text { even }, \\ \mathbb{Z}_{2}^{e f} \oplus \mathbb{Z} / w_{i}(E) & \text { for } n=2 i-1>1 \text { odd },\end{cases}
$$

and

$$
K_{n}\left(\mathcal{O}_{E} ; \mathbb{Z}_{2}\right) \cong \begin{cases}\mathbb{Z}_{2} & \text { for } n=0, \\ \mathbb{Z}_{2}^{\text {ef }} \oplus \mathbb{Z} / w_{i}(E) & \text { for } n=2 i-1 \text { odd } \\ \mathbb{Z} / w_{i}(E) & \text { for } n=2 i>0 \text { even }\end{cases}
$$

Proof. Theorem 1.12 and Proposition 3.6 give a calculation of $K_{n}\left(E ; \mathbb{Z} / 2^{\infty}\right)$ for all $n$. The formulas for $K_{n}\left(E ; \mathbb{Z}_{2}\right)$ follow by the universal coefficient theorem.

Let $k_{\mathfrak{P}}$ be as above. By Quillen's calculation we have $K_{n}\left(k_{\mathfrak{P}} ; \mathbb{Z} / 2^{\infty}\right)=0$ for $n \neq 0$, since $k_{\mathfrak{P}}$ has characteristic 2 . Thus the $K$-theory localization sequence degenerates to give a calculation of $K_{n}\left(\mathcal{O}_{E} ; \mathbb{Z} / 2^{\infty}\right)$. The expression given for $K_{n}\left(\mathcal{O}_{E} ; \mathbb{Z}_{2}\right)$ then follows by the universal coefficient theorem.

Example 3.8. When $E=\mathbb{Q}_{2}$ is the 2-adic rationals, then $w_{i}\left(\mathbb{Q}_{2}\right)=2$ for $i$ odd and $w_{i}\left(\mathbb{Q}_{2}\right)=2^{2+\log _{2}(i)}$ for $i$ even by Proposition 1.9(b), (c). In this case we recover the calculations of $[R, 0.1]$ :

$$
K_{n}\left(\mathbb{Z}_{2} ; \mathbb{Z}_{2}\right) \cong \begin{cases}\mathbb{Z}_{2} & \text { for } n=0 \\ \mathbb{Z}_{2} \oplus \mathbb{Z} / w_{i}\left(\mathbb{Q}_{2}\right) & \text { for } n=2 i-1>0 \text { odd } \\ \mathbb{Z} / w_{i}\left(\mathbb{Q}_{2}\right) & \text { for } n=2 i>0 \text { even. }\end{cases}
$$

This result was obtained in $[R]$ by different methods, involving the cyclotomic trace map to topological cyclic homology. Those topological methods also give the improvement

$$
K_{n}\left(\mathbb{Q}_{2} ; \mathbb{Z} / 2\right) \cong \begin{cases}\mathbb{Z} / 2 & \text { for } n=0, \\ \mathbb{Q}_{2}^{\times} / 2 \cong(\mathbb{Z} / 2)^{3} & \text { for } n=2 i-1>0 \text { odd }, \\ \mathbb{Z} / 4 & \text { for } n \equiv 2 \bmod 4, n>0, \\ \mathbb{Z} / 2 \oplus \mathbb{Z} / 2 & \text { for } n \equiv 0 \bmod 4, n>0,\end{cases}
$$

on Theorem 1.15 (see $[\mathrm{R}, 4.2]$ ). The topologically explicit generators of these groups will be used to describe the spectral sequence for the reals in section 5 .

\section{4. ÉtAle COHOMOLOGy OF GLOBAL FIELDS}

In order to use the spectral sequence (1.3) we need to have control of $H^{n}(F ; W(i))$ and $H^{n}(R ; W(i))$ for $i \geq 2$. For $n=0$ we have $H^{0}(R ; W(i)) \cong H^{0}(F ; W(i)) \cong$ $\mathbb{Z} / w_{i}(F)$ by Propositions 1.8 and 2.9(b). Otherwise the results we need are consequences of Tate's duality theory for global fields, which is the topic of this section. We refer to Milne's book [M2] on arithmetic duality theorems for further details.

We remark that the groups $H^{1}(R ; W(i))$ are not completely known. We will determine the rank and number of finite cyclic summands in the Pontryagin dual of 
$H^{1}(R ; W(i))$ in Propositions 6.12 and 6.13, using the Global Duality Theorem 4.9. This leaves only the order of the cyclic summands in $H^{1}(R ; W(i))$ as mysterious.

Definition 4.1. Let $F$ be a number field, with $r_{1}$ real embeddings. We say that $F$ is real when $r_{1}>0$, and that $F$ is totally imaginary when $r_{1}=0$. Let

$$
\alpha^{n}(F ; M): H^{n}(F ; M) \rightarrow \bigoplus^{r_{1}} H^{n}(\mathbb{R} ; M)
$$

be the sum of the homomorphisms induced by the $r_{1}$ real embeddings $F \rightarrow \mathbb{R}$ of $F$. Likewise let

$$
\alpha^{n}(R ; M): H^{n}(R ; M) \rightarrow \bigoplus^{r_{1}} H^{n}(\mathbb{R} ; M)
$$

be the sum of the homomorphisms induced by the $r_{1}$ composites $R \rightarrow F \rightarrow \mathbb{R}$.

The following result of Tate appears as [T, 3.1(c)] and as [M2, I.4.10(c)].

Theorem 4.2 (Tate). For $n \geq 3$ there are natural isomorphisms

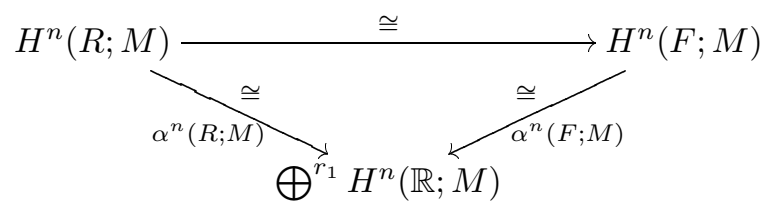

for $M$ finite, $M=W(i)$ or $M=\mathbb{Z}_{2}(i)$.

Proof. For $M$ finite this follows from the references cited, using the set of places $S_{\infty}$ in the case of $\alpha^{n}(R ; M)$, and the set of all places $\Sigma$ in the case of $\alpha^{n}(F ; M)$. The claims for $M=W(i)$ and $M=\mathbb{Z}_{2}(i)$ then follow by passage to colimits and limits, respectively.

Lemma 4.3. For $n \geq 0$ and any integer $i$ we have

$$
H^{n}(\mathbb{R} ; W(i))= \begin{cases}\mathbb{Z} / 2^{\infty} & \text { for } n=0 \text { and } i \text { even, } \\ \mathbb{Z} / 2 & \text { for } i-n \text { odd }, \\ 0 & \text { otherwise. }\end{cases}
$$

Also $H^{n}(\mathbb{R} ; \mathbb{Z} / 2) \cong \mathbb{Z} / 2$ for $n \geq 0$.

Proof. This is a straightforward calculation of group cohomology using the 2periodic resolution for $\operatorname{Gal}(\mathbb{C} / \mathbb{R}) \cong C_{2}$. See $[\mathrm{W} 1,7.1 .1]$.

Corollary 4.4. For $n \geq 3$ and all integers $i$, the maps $\alpha^{n}$ are isomorphisms:

$$
H^{n}(R ; \mathbb{Z} / 2) \cong H^{n}(F ; \mathbb{Z} / 2) \cong(\mathbb{Z} / 2)^{r_{1}}
$$

and

$$
H^{n}(R ; W(i)) \cong H^{n}(F ; W(i)) \cong \begin{cases}(\mathbb{Z} / 2)^{r_{1}} & \text { for } i-n \text { odd }, \\ 0 & \text { for } i-n \text { even } .\end{cases}
$$

Proof. See [W1, 7.1], or use Theorem 4.2 above.

Theorem 4.5 (Soulé, Weibel). (a) If $F$ is totally imaginary and $i \geq 2$, then $H^{2}(R ; W(i))=H^{2}(F ; W(i))=0$.

(b) If $F$ is real and $i \geq 2$, then $H^{2}(R ; W(i))$ and $H^{2}(F ; W(i))$ have exponent 2 . 
Proof. Part (a) is [W1, 7.3].

Part (b) follows, since the natural map $H^{2}(F ; W(i)) \rightarrow H^{2}(F(\sqrt{-1}) ; W(i))$ followed by the norm map (transfer) going back induces multiplication by 2 , and factors through a trivial group by part (a). The argument for $R$ is similar. See $[\mathrm{W} 1,7.2 .1]$.

Proposition 4.6. Let $F$ be a number field. Then for $i \geq 2$

$$
\alpha^{2}: H^{2}(R ; W(i)) \cong H^{2}(F ; W(i)) \cong \begin{cases}(\mathbb{Z} / 2)^{r_{1}} & \text { for } i \text { odd }, \\ 0 & \text { for } i \text { even. }\end{cases}
$$

Proof. We use Theorem 4.5 and the Kummer sequence

$$
\stackrel{2}{\rightarrow} H^{2}(F ; W(i)) \rightarrow H^{3}(F ; \mathbb{Z} / 2) \rightarrow H^{3}(F ; W(i)) \stackrel{2}{\rightarrow}
$$

where both maps labeled 2 are equal to zero for $i \geq 2$. By Corollary 4.4 we have $H^{3}(F ; \mathbb{Z} / 2) \cong(\mathbb{Z} / 2)^{r_{1}}$, while $H^{3}(F ; W(i))=0$ for $i$ odd, and $H^{3}(F ; W(i)) \cong$ $(\mathbb{Z} / 2)^{r_{1}}$ for $i$ even. Hence by exactness $H^{2}(F ; W(i)) \cong(\mathbb{Z} / 2)^{r_{1}}$ for $i$ odd, and $H^{2}(F ; W(i))=0$ for $i$ even. The argument for $R$ is identical.

Hence Corollary 4.4 also applies with $n=2$ and $i \geq 2$. The case $i=1$ is described in Proposition 2.7(b). For $i=0$ it is known (see Satz 4.6 and Lemma 7.1 of [Sc]) that the vanishing of $H^{2}(R ; W(0))$ is equivalent to Leopoldt's conjecture, which holds if $F$ is Abelian by Corollary 5.32 of [Ws].

Proposition 4.7. Let $F$ be a number field, $R$ its ring of 2-integers, and suppose $i \geq 1$. The étale cohomology localization sequence $2.9(b)$ for $R$ breaks up into short exact sequences

$$
0 \rightarrow H^{1}(R ; W(i)) \rightarrow H^{1}(F ; W(i)) \stackrel{\partial^{e}}{\longrightarrow} \bigoplus_{\mathfrak{P} \nmid \ell} H^{0}\left(k_{\mathfrak{P}} ; W(i-1)\right) \rightarrow 0
$$

and isomorphisms $H^{n}(R ; W(i)) \rightarrow H^{n}(F ; W(i))$ for $n \neq 1,2$, as well as for $n=2$ and $i \geq 2$.

Proof. For $i \geq 2$ this is immediate from the isomorphism in Proposition 4.6. For $i=1$, use the injection $H^{2}(R ; W(1)) \rightarrow H^{2}(F ; W(1))$. (See the note following (2.6) and Proposition 2.7(b).)

We remark that Proposition 4.7 also holds with 2 replaced by an odd prime $\ell$. See [So, p. 287].

Definition 4.8. When $E$ is a local field let $\hat{H}^{n}(E ; M)$ be the Tate cohomology groups of the absolute Galois group $G_{E}$ with coefficients in $M$. Thus $\hat{H}^{n}(E ; M)=$ $H^{n}(E ; M)$ when $n>0$, and $\hat{H}^{0}(E ; M)=H^{0}(E ; M)$ when $E$ is non-Archimedean. In the Archimedean cases we have

$$
\hat{H}^{0}(\mathbb{R} ; M)=\hat{H}^{0}(\operatorname{Gal}(\mathbb{C} / \mathbb{R}) ; M)=M^{\operatorname{Gal}(\mathbb{C} / \mathbb{R})} / N_{\mathbb{C} / \mathbb{R}}(M),
$$

while $\hat{H}^{0}(\mathbb{C} ; M)=0$. Concretely, for $\ell=2$ we have $\hat{H}^{0}(\mathbb{R} ; \mathbb{Z} / 2) \cong \mathbb{Z} / 2$, and

$$
\hat{H}^{0}(\mathbb{R} ; W(i)) \cong \begin{cases}0 & \text { for } i \text { even } \\ \mathbb{Z} / 2 & \text { for } i \text { odd }\end{cases}
$$


Recall that $S_{\infty}$ denotes the set of primes in $F$ dividing $\ell$, together with the Archimedean places. This is a finite set. Let

$$
\beta^{n}(R ; M): H^{n}(R ; M) \rightarrow \bigoplus_{v \in S_{\infty}} \hat{H}^{n}\left(F_{v} ; M\right)
$$

be the sum of the homomorphisms induced by the completion maps $R \rightarrow F \rightarrow F_{v}$, for all places $v$ in $S_{\infty}$.

When $E$ is a local field and $M$ is an infinite $G_{E}$-module, e.g. $M=W(i)$ or $\mathbb{Z}_{\ell}(i)$, we extend the definition preceding Theorem 2.11 by letting $M^{\prime}=\operatorname{Hom}(M, \mu)$, where $\mu$ is the torsion subgroup of $\mathbb{G}_{m}$. In particular $W(i)^{\prime} \cong \mathbb{Z}_{\ell}(1-i)$ and $\mathbb{Z}_{\ell}(i)^{\prime} \cong W(1-i)$.

With this notation, local Tate-Poitou duality induces isomorphisms

$$
\hat{H}^{n}\left(F_{v} ; M\right) \cong \hat{H}^{2-n}\left(F_{v} ; M^{\prime}\right)^{\#}
$$

for all $n$, by Theorem 2.11 and Corollary 2.12. (The omitted Archimedean cases are straightforward.) Let

$$
\gamma^{n}(R ; M): \bigoplus_{v \in S_{\infty}} \hat{H}^{n}\left(F_{v} ; M\right) \rightarrow H^{2-n}\left(R ; M^{\prime}\right)^{\#}
$$

be the direct sum of these local Tate-Poitou duality isomorphisms composed with the Pontryagin dual of the map $\beta^{2-n}\left(R ; M^{\prime}\right)$.

Let $\amalg^{n}(R ; M)=\operatorname{ker} \beta^{n}(R ; M)$. When $n=1$ this is the Tate-Shafarevich group.

We now state the remainder of [T, 3.1], or [M2, I.4.10]. The pairing in question is described immediately before the cited theorem in [T], and on page 79 of [M2]. In the statement of (b) we briefly write $\beta^{n}=\beta^{n}(R ; M)$ and $\gamma^{n}=\gamma^{n}(R ; M)$.

Theorem 4.9 (Tate). (a) There is a natural perfect pairing

$$
\amalg^{n}(R ; M) \times \amalg^{3-n}\left(R ; M^{\prime}\right) \rightarrow \mathbb{Q} / \mathbb{Z}
$$

for $n=1,2$ and for $M$ finite, $M=W(i)$ or $M=\mathbb{Z}_{\ell}(i)$. These groups are finite when $M$ is finite, discrete torsion groups when $M=W(i)$, and profinite when $M=\mathbb{Z}_{\ell}(i)$.

(b) There is a natural 9-term exact sequence

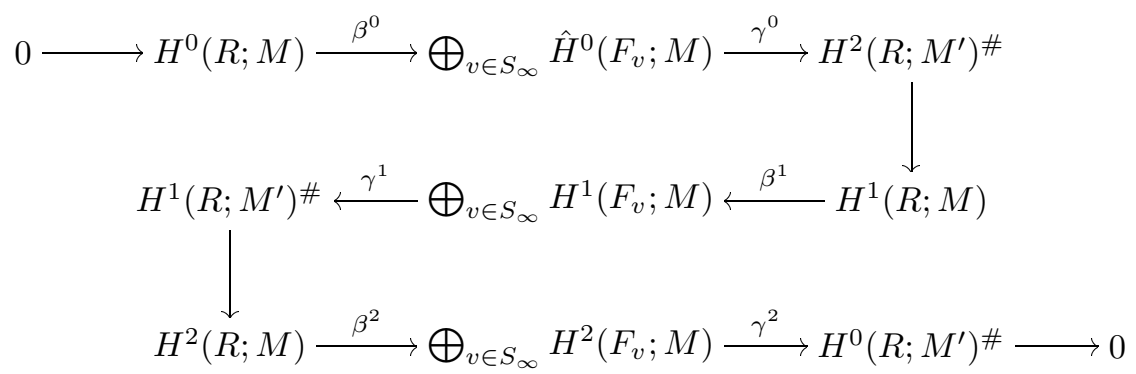

for $M$ finite, $M=W(i)$ or $M=\mathbb{Z}_{\ell}(i)$. Hence $H^{n}(R ; M)$ is finite when $M$ is finite, a discrete torsion group when $M=W(i)$, and profinite when $M=\mathbb{Z}_{\ell}(i)$.

Proof. In (a), the naturality of the pairings with respect to the transition maps $\iota_{*}$ and $\pi_{*}$ in the systems defining $\amalg^{n}(R ; W(i))$ and $\amalg^{3-n}\left(R ; \mathbb{Z}_{\ell}(1-i)\right)$ follows as in the proof of Corollary 2.12 above. The claims for $M=W(i)$ and $M=\mathbb{Z}_{\ell}(i)$ then follow from the cases with $M$ finite, by exactness of colimits of discrete torsion groups and of limits of profinite groups. (Recall Lemma 2.2.) 
The naturality of the pairings above suffices to prove that the 9-term exact sequence in (b) is natural with respect to $\iota$ and $\pi$. Thus again the claims with $M=W(i)$ or $M=\mathbb{Z}_{\ell}(i)$ follow from the finite cases by passage to colimits or limits.

Theorem 4.10. Let $F$ be a number field, and $R$ its ring of $\ell$-integers. Suppose $i \geq 1$. Then $\amalg^{2}(R ; W(i))=0$, so $\beta^{2}(R ; W(i))$ is injective and $\gamma^{1}(R ; W(i))$ is surjective.

Hence Tate's 9-term exact sequence breaks up into an exact 6-term sequence and a short exact sequence.

Proof. We first prove that $\beta^{2}=\beta^{2}(R ; W(i))$ is injective for $i \geq 2$. By Theorem 4.5 and Proposition 4.6 we have $H^{2}(R ; W(i))=0$ if $\ell \neq 2$, if $F$ is totally imaginary or if $i$ is even. In these cases $\beta^{2}$ is obviously injective. Otherwise assume that $\ell=2$, $F$ is real and $i$ is odd, so that $H^{2}(R ; W(i)) \cong(\mathbb{Z} / 2)^{r_{1}}$. Since $H^{2}\left(F_{v} ; W(i)\right)=0$ for each complex or non-Archimedean place of $F$, we also have $\bigoplus_{v \in S_{\infty}} H^{2}\left(F_{v} ; W(i)\right) \cong$ $(\mathbb{Z} / 2)^{r_{1}}$. And $H^{0}\left(R ; \mathbb{Z}_{2}(1-i)\right)=0$ for $i \neq 1$, so $\beta^{2}$ is a surjection between abstractly isomorphic finite groups, thus an isomorphism.

When $i=1$ the maps $\beta^{2}$ and $\gamma^{2}$ are readily identified with the 2-components of the Brauer-Hasse-Noether short exact sequence (2.6) for $\operatorname{Br}(R)$. In particular $\beta^{2}$ is injective.

\section{The SPECTRAL SEQUenCE FOR THE REAL NUmbers}

We now consider the mod 2 and mod $2^{\infty}$ spectral sequences for the field $\mathbb{R}$ of real numbers. These do not collapse at the $E_{2}$-term, and there are nontrivial additive extensions. These results about the spectral sequence for $\mathbb{R}$ will form the basis of our calculations for real number fields in section 6 . They also provide the needed justification for Proposition 4 in [W3].

The $E_{2}$-terms of these spectral sequences are given by Lemma 4.3.

$$
E_{2}^{p, q}=\left\{\begin{array}{ll}
\mathbb{Z} / 2 & \text { for } q \leq p \leq 0, \\
0 & \text { otherwise }
\end{array} \Longrightarrow K_{-p-q}(\mathbb{R} ; \mathbb{Z} / 2)\right.
$$

and

$$
E_{2}^{p, q}= \begin{cases}\mathbb{Z} / 2^{\infty} & \text { for } q=p \leq 0 \text { and } p \text { even, } \\ \mathbb{Z} / 2 & \text { for } q \leq p \leq 0 \text { and } p \text { odd, } \Longrightarrow K_{-p-q}\left(\mathbb{R} ; \mathbb{Z} / 2^{\infty}\right) . \\ 0 & \text { otherwise, }\end{cases}
$$

To formulate our theorem about the mod 2 spectral sequence for the reals, we need to recall some well known elements in stable homotopy.

Notation 5.1. Define $i_{1}: \mathbb{Z} \rightarrow \mathbb{Z} / 2$ and $\iota_{1}: \mathbb{Z} / 2 \rightarrow \mathbb{Z} / 2^{\infty}$ as the surjective and injective group homomorphisms, respectively. These fit into a map of short exact sequences

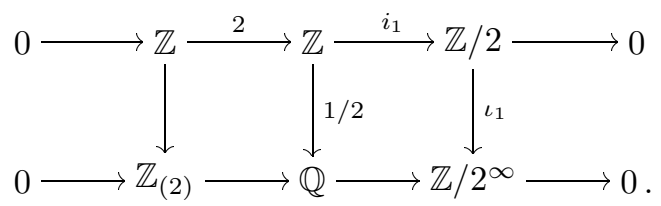


Also let $i_{1}: \pi_{*}(X) \rightarrow \pi_{*}(X ; \mathbb{Z} / 2)$ and $\iota_{1}: \pi_{*}(X ; \mathbb{Z} / 2) \rightarrow \pi_{*}\left(X ; \mathbb{Z} / 2^{\infty}\right)$ denote the induced homomorphisms for spectra $X$.

Let $\mu_{8 k+1} \in \pi_{8 k+1}\left(Q S^{0}\right)$ and $\eta \mu_{8 k+1} \in \pi_{8 k+2}\left(Q S^{0}\right)$ denote Adams' $\mu$-elements [A, p. 68]. These generate direct $\mathbb{Z} / 2$-summands in their respective homotopy groups, and are detected (i.e., have nonzero images) in $K_{8 k+1}(\mathbb{R})$ and $K_{8 k+2}(\mathbb{R})$ via the unit map $Q S^{0} \rightarrow K(\mathbb{R})$.

Let $\beta_{8 k+2} \in \pi_{8 k+2}\left(Q S^{0} ; \mathbb{Z} / 2\right)$ denote a preimage of $\mu_{8 k+1}$ under the mod 2 Bockstein map

$$
\pi_{8 k+2}\left(Q S^{0} ; \mathbb{Z} / 2\right) \rightarrow \pi_{8 k+1}\left(Q S^{0}\right) .
$$

Then $\iota_{1}\left(\beta_{8 k+2}\right) \in \pi_{8 k+2}\left(Q S^{0} ; \mathbb{Z} / 2^{\infty}\right)$ is a preimage of $\mu_{8 k+1}$ under the mod $2^{\infty}$ Bockstein map

$$
\pi_{8 k+2}\left(Q S^{0} ; \mathbb{Z} / 2^{\infty}\right) \rightarrow \pi_{8 k+1}\left(Q S^{0}\right)\{2\}=\pi_{8 k+1}\left(Q S^{0} ; \mathbb{Z}_{(2)}\right),
$$

as is seen from the map of short exact sequences above.

Let $\beta_{8 k} \in \pi_{8 k}\left(Q S^{0} ; \mathbb{Z} / 2\right)$ represent the image under the $k$-fold Adams $v_{1}^{4}$-action [A] on the generator of $\pi_{0}\left(Q S^{0} ; \mathbb{Z} / 2\right) \cong \mathbb{Z} / 2$. This class maps to the generators of $K_{8 k}(\mathbb{R} ; \mathbb{Z} / 2) \cong K_{8 k}(\mathbb{C} ; \mathbb{Z} / 2) \cong \mathbb{Z} / 2$ under the unit maps $Q S^{0} \rightarrow K(\mathbb{R}) \rightarrow K(\mathbb{C})$.

Then $\beta_{8 k}, i_{1}\left(\mu_{8 k+1}\right), \beta_{8 k+2}, \eta \beta_{8 k+2}$ and $\eta^{2} \beta_{8 k+2}$ in $\pi_{*}\left(Q S^{0} ; \mathbb{Z} / 2\right)$ are classes that map to generators of $K_{*}(\mathbb{R} ; \mathbb{Z} / 2)$. In addition, the additive extension in $K_{8 k+2}(\mathbb{R} ; \mathbb{Z} / 2) \cong \mathbb{Z} / 4$ corresponds to the relation $2 \cdot \beta_{8 k+2}=i_{1}\left(\eta \mu_{8 k+1}\right)$.

Also the classes $\iota_{1}\left(\beta_{8 k+2}\right)$ and $\iota_{1}\left(\eta \beta_{8 k+2}\right)$ in $\pi_{*}\left(Q S^{0} ; \mathbb{Z} / 2^{\infty}\right)$ map to generators of $K_{*}\left(\mathbb{R} ; \mathbb{Z} / 2^{\infty}\right)$ in degrees $8 k+2$ and $8 k+3$. In degree $8 k+4, \iota_{1}\left(\eta^{2} \beta_{8 k+2}\right)$ maps to the order 2 element in $K_{8 k+4}\left(\mathbb{R} ; \mathbb{Z} / 2^{\infty}\right) \cong \mathbb{Z} / 2^{\infty}$. The remainder of $K_{8 k+4}\left(\mathbb{R} ; \mathbb{Z} / 2^{\infty}\right)$ is detected by the natural map onto $K_{8 k+4}\left(\mathbb{C} ; \mathbb{Z} / 2^{\infty}\right) \cong \mathbb{Z} / 2^{\infty}$, which multiplies by 2 . Finally, in degree $8 k$ we have $K_{8 k}\left(\mathbb{R} ; \mathbb{Z} / 2^{\infty}\right) \cong K_{8 k}\left(\mathbb{C} ; \mathbb{Z} / 2^{\infty}\right)$.

We shall need the following topological result, which is a restatement of part of $[\mathrm{R}, 4.2(\mathrm{c})$ and $4.3(\mathrm{c})]$. Recall from Theorem 1.15 above that for $i>0$ we have $K_{2 i-1}\left(\mathbb{Q}_{2} ; \mathbb{Z} / 2\right) \cong H^{1}\left(\mathbb{Q}_{2} ; \mathbb{Z} / 2\right) \cong(\mathbb{Z} / 2)^{3}$, while $K_{2 i}\left(\mathbb{Q}_{2} ; \mathbb{Z} / 2\right)$ is an extension of $H^{0}\left(\mathbb{Q}_{2} ; \mathbb{Z} / 2\right)=\mathbb{Z} / 2$ by $H^{2}\left(\mathbb{Q}_{2} ; \mathbb{Z} / 2\right)=\mathbb{Z} / 2$.

Lemma 5.2. The images of $i_{1}\left(\mu_{8 k+1}\right)$ and $\eta \beta_{8 k+2}$ are nonzero in $K_{8 k+1}\left(\mathbb{Q}_{2} ; \mathbb{Z} / 2\right)$, resp. $K_{8 k+3}\left(\mathbb{Q}_{2} ; \mathbb{Z} / 2\right)$.

The images of $i_{1}\left(\eta \mu_{8 k+1}\right)$ and $\beta_{8 k+2}$ are nonzero in $K_{8 k+2}\left(\mathbb{Q}_{2} ; \mathbb{Z} / 2\right) \cong \mathbb{Z} / 4$, with $2 \cdot \beta_{8 k+2}=i_{1}\left(\eta \mu_{8 k+1}\right)$.

The image of $\eta^{2} \beta_{8 k+2}$ is nonzero in $K_{8 k+4}\left(\mathbb{Q}_{2} ; \mathbb{Z} / 2\right) \cong \mathbb{Z} / 2 \oplus \mathbb{Z} / 2$.

Proof. When $k=0$, this is standard. In the notation of $[\mathrm{R}, 4.2(\mathrm{c})]$ we have: $i_{1}\left(\mu_{1}\right)=i_{1}(\eta), \beta_{2}=\tilde{\eta}_{2}$ and $\eta \beta_{2}=\eta \tilde{\eta}_{2}$ are detected as $\xi_{1}, \xi_{2}$ and $\xi_{3}$, respectively, while $i_{1}\left(\eta \mu_{1}\right)=i_{1}\left(\eta^{2}\right)$ is detected by $\partial\left(\xi_{3}(0)\right)$. From this, one calculates that $\eta^{2} \beta_{2}=\eta^{2} \tilde{\eta}_{2}$ is detected by $\partial\left(\xi_{5}(0)\right)$, using the formulas $\partial(\lambda)=\eta^{2}, \lambda \tilde{\eta}_{2}=i_{1}(\kappa)$ and $i_{1}(\partial(\kappa))=\partial\left(\xi_{5}(0)\right)$.

By $[\mathrm{R}, 4.3(\mathrm{c})]$, Adams' element $v_{1}^{4}$ acts injectively on $K_{*}\left(\mathbb{Q}_{2} ; \mathbb{Z} / 2\right)$. Hence this pattern of detection repeats in higher degrees.

Theorem 5.3. In the mod 2 Bloch-Lichtenbaum spectral sequence for $\mathbb{R}$ the classes $\beta_{8 k}, i_{1}\left(\mu_{8 k+1}\right), i_{1}\left(\eta \mu_{8 k+1}\right), \beta_{8 k+2}, \eta \beta_{8 k+2}$ and $\eta^{2} \beta_{8 k+2}$ are represented by permanent cycles in bidegrees $(p, q)$, with $p=-4 k$ or $p=-4 k-1$, and $0 \leq p-q \leq 2$, for all $k \geq 0$. 
The remaining classes do not survive to the $E_{\infty}$-term. In particular, the classes in bidegrees $(p, q)$ with $p=-4 k-2$ or $p=-4 k-3$ and $0 \leq p-q \leq 4$ support nontrivial differentials.

There is a nontrivial additive extension $\mathbb{Z} / 2 \rtimes \mathbb{Z} / 2 \cong \mathbb{Z} / 4$ in total degree $-(8 k+2)$.

\begin{tabular}{|c|c|c|c|}
\hline & & & $\beta_{8 k}$ \\
\hline & & $\beta_{8 k+2}$ & $i_{1}\left(\mu_{8 k+1}\right)$ \\
\hline & 0 & $\eta \beta_{8 k+2}$ & $i_{1}\left(\eta \mu_{8 k+1}\right)$ \\
\hline 0 & 0 & $\eta^{2} \beta_{8 k+2}$ & 0 \\
\hline
\end{tabular}

Proof. The classes $\beta_{8 k}$ and $\beta_{8 k+2}$ have complex Adams e-invariant $-1 / 2$, hence are detected in $K_{*}(\mathbb{C} ; \mathbb{Z} / 2)$. Thus the classes in bidegrees $(-4 k,-4 k)$ and $(-4 k-1,-4 k-1)$ are permanent cycles, representing the images of $\beta_{8 k}$ and $\beta_{8 k+2}$.

We next turn to the classes $i_{1}\left(\mu_{8 k+1}\right)$ and $\eta \beta_{8 k+2}$, referring to these as the first and second case, respectively. In both cases consider the following diagram, with $i=4 k+1$ or $i=4 k+2$, respectively. Here $p$ is any prime, and the maps labeled "edge" are the edge maps in the mod 2 Bloch-Lichtenbaum spectral sequences. The right hand edge map is an isomorphism by Theorem 1.15.

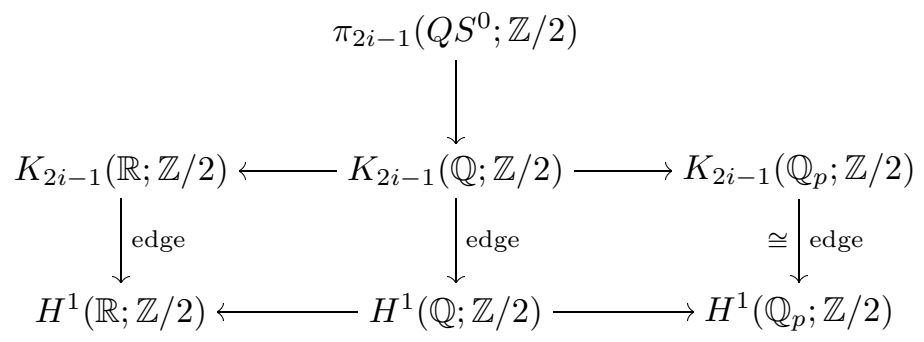

Let $x \in H^{1}(\mathbb{Q} ; \mathbb{Z} / 2)$ denote the image of $i_{1}\left(\mu_{8 k+1}\right)$, resp. $\eta \beta_{8 k+2}$, under the composed vertical map. For each prime $p$ let $x_{p} \in H^{1}\left(\mathbb{Q}_{p} ; \mathbb{Z} / 2\right)$ and $z \in H^{1}(\mathbb{R} ; \mathbb{Z} / 2) \cong$ $\mathbb{Z} / 2$ be the further images of $x$, under the natural maps.

We must prove that $z \neq 0$, for then $i_{1}\left(\mu_{8 k+1}\right)$, resp. $\eta \beta_{8 k+2}$, maps to a class represented as a permanent cycle in bidegree $(1-i,-i)$, as asserted.

Lemma 5.4. In both cases, $x$ and $z$ are the image of a nonzero $y \in H^{1}\left(\mathbb{Z}\left[\frac{1}{2}\right] ; \mathbb{Z} / 2\right)$. Moreover, $\iota_{1}(y) \neq 0$ in $H^{1}\left(\mathbb{Z}\left[\frac{1}{2}\right] ; W(i)\right)$ in the second case.

Proof. In both cases we know by Lemma 5.2 that $x_{2} \neq 0$ and hence $x \neq 0$, so if $y$ exists it is nonzero. Recall the étale cohomology localization sequence 2.9(b)

$$
0 \rightarrow H^{1}\left(\mathbb{Z}\left[\frac{1}{2}\right] ; \mathbb{Z} / 2\right) \rightarrow H^{1}(\mathbb{Q} ; \mathbb{Z} / 2) \stackrel{\partial^{e}}{\longrightarrow} \bigoplus_{p \neq 2} H^{0}\left(\mathbb{F}_{p} ; \mathbb{Z} / 2\right)
$$

for $\mathbb{Z}\left[\frac{1}{2}\right] \rightarrow \mathbb{Q}$. Comparing with the localization sequence 2.9 (a) for $\mathbb{Z}_{p} \rightarrow \mathbb{Q}_{p}$, we see that the map $\partial^{e}$ is the direct sum of the composite maps

$$
H^{1}(\mathbb{Q} ; \mathbb{Z} / 2) \rightarrow H^{1}\left(\mathbb{Q}_{p} ; \mathbb{Z} / 2\right) \stackrel{\partial_{p}^{e}}{\longrightarrow} H^{0}\left(\mathbb{F}_{p} ; \mathbb{Z} / 2\right)
$$


for all primes $p \neq 2$. Hence it suffices to prove that $\partial_{p}^{e}\left(x_{p}\right)=0$ for all $p \neq 2$. For this, we consider the following diagram:



The vertical maps are induced by the coefficient injection $\iota_{1}: \mathbb{Z} / 2 \rightarrow W(i)$. The right hand vertical map is injective because $H^{0}$ is left exact.

In the first case, the image $\iota_{1}(x)$ of $\mu_{8 k+1} \in \pi_{8 k+1}\left(Q S^{0}\right)$ in $H^{1}(\mathbb{Q} ; W(i))$ factors through $\pi_{8 k+1}\left(Q S^{0} ; \mathbb{Q}\right)=0$, hence is zero. Thus also $\iota_{1}\left(x_{p}\right)=0$ and $\partial_{p}^{e}\left(x_{p}\right)=0$ for all primes $p$ (including $p=2$ ), by injectivity of the right hand vertical map $\iota_{1}$.

In the second case, the map from $\pi_{8 k+3}\left(Q S^{0} ; \mathbb{Z} / 2\right)$ to $H^{1}\left(\mathbb{Q}_{p} ; W(i)\right)$ factors through $K_{8 k+3}\left(\mathbb{Z}_{p} ; \mathbb{Z} / 2^{\infty}\right)$ under the unit map $Q S^{0} \rightarrow K\left(\mathbb{Z}_{p}\right) \rightarrow K\left(\mathbb{Q}_{p}\right)$. But

$$
K_{8 k+3}\left(\mathbb{Z}_{p} ; \mathbb{Z} / 2^{\infty}\right) \cong K_{8 k+2}\left(\mathbb{Z}_{p} ; \mathbb{Z}_{2}\right) \cong K_{8 k+2}\left(\mathbb{F}_{p} ; \mathbb{Z}_{2}\right)=0
$$

for all primes $p \neq 2$ by Gabber's Rigidity Theorem 3.2. Thus also in this case $\iota_{1}\left(x_{p}\right)=0$ and $\partial_{p}^{e}\left(x_{p}\right)=0$ for all primes $p \neq 2$.

For the last claim, note that in the second case $\iota_{1}\left(x_{2}\right)$ has nonzero $\bmod 2^{\infty}$ Bockstein, so $\iota_{1}(x) \neq 0$ and thus $\iota_{1}(y) \neq 0$.

In the first case, regarding $i_{1}\left(\mu_{8 k+1}\right)$, we conclude with the following diagram with $i=4 k+1$. Since $i$ is odd, $H^{0}\left(\mathbb{Q}_{2} ; W(i)\right)=H^{0}\left(\mathbb{Z}\left[\frac{1}{2}\right] ; W(i)\right)=H^{0}(\mathbb{R} ; W(i))=$ $\mathbb{Z} / 2$ by Proposition $1.9(\mathrm{~b})$ and $H^{1}(\mathbb{R} ; W(i))=0$ by Lemma 4.3 .

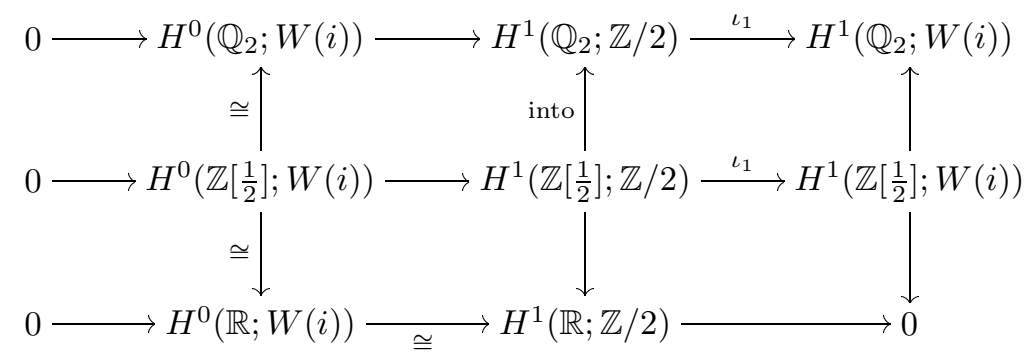

The rows are exact, and the vertical maps are induced by ring homomorphisms. Also the group $H^{1}\left(\mathbb{Z}\left[\frac{1}{2}\right] ; \mathbb{Z} / 2\right) \cong \mathbb{Z}\left[\frac{1}{2}\right]^{\times} / 2 \cong(\mathbb{Z} / 2)^{2}$, which is generated by 2 and -1 , injects into $H^{1}\left(\mathbb{Q}_{2} ; \mathbb{Z} / 2\right) \cong \mathbb{Q}_{2}^{\times} / 2 \cong(\mathbb{Z} / 2)^{3}$, which is generated by $2,-1$ and 5 . We chose $y$ to map to $z$, as well as to $x_{2} \neq 0$. Since we saw in the proof of Lemma 5.4 that $\iota_{1}\left(x_{2}\right)=0$, a diagram chase shows that $z$ is nonzero. This concludes the proof of Theorem 5.3 for $i_{1}\left(\mu_{8 k+1}\right)$.

In the second case, regarding $\eta \beta_{8 k+2}$, we use the commutative square below:

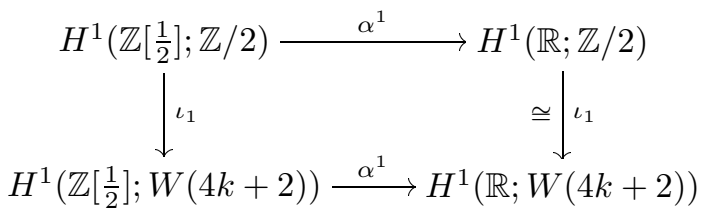

Since $\iota_{1}(x) \neq 0$ in this case, we have $\iota_{1}(y) \neq 0$. By the following lemma, it then follows that $\iota_{1}(z) \neq 0$ and $z \neq 0$, concluding the proof of Theorem 5.3 for $\eta \beta_{8 k+2}$. 
Lemma 5.5. For all even $i \neq 0$, the natural map

$$
\alpha^{1}: H^{1}\left(\mathbb{Z}\left[\frac{1}{2}\right] ; W(i)\right) \rightarrow H^{1}(\mathbb{R} ; W(i)) \cong \mathbb{Z} / 2
$$

is an isomorphism.

Proof. Associated to $\iota_{1}: \mathbb{Z} / 2 \rightarrow W(i)$ we have $H^{1}(\mathbb{R} ; \mathbb{Z} / 2) \cong H^{1}(\mathbb{R} ; W(i)) \cong \mathbb{Z} / 2$ by Lemma 4.3 , and a commutative diagram



Here the middle vertical map is onto by the Kummer sequence: $H^{1}\left(\mathbb{Z}\left[\frac{1}{2}\right] ; \mathbb{Z} / 2\right) \cong$ $\mathbb{Z}\left[\frac{1}{2}\right]^{\times} / 2 \cong(\mathbb{Z} / 2)^{2}$ has generators -1 and 2 , while $H^{1}(\mathbb{R} ; \mathbb{Z} / 2) \cong \mathbb{R}^{\times} / 2 \cong \mathbb{Z} / 2$ is generated by -1 . Since $i \neq 0$ we also have $H^{0}\left(\mathbb{Z}\left[\frac{1}{2}\right] ; W(i)\right) / 2 \cong \mathbb{Z} / 2$ by Propositions 1.8 and 1.9. By the 5-lemma, we have an isomorphism ${ }_{2} H^{1}\left(\mathbb{Z}\left[\frac{1}{2}\right] ; W(i)\right) \cong H^{1}(\mathbb{R} ; W(i))$ and $\alpha^{1}$ is a split surjection.

Now if $A$ is any 2-torsion Abelian group such that ${ }_{2} A$ is a summand of $A$, then $A \cong{ }_{2} A$. Applying this to $A=H^{1}\left(\mathbb{Z}\left[\frac{1}{2}\right] ; W(i)\right)$ yields the lemma.

We proceed with the proof of Theorem 5.3, regarding the image of $i_{1}\left(\eta \mu_{8 k+1}\right)$ and $\eta^{2} \beta_{8 k+2}$ in the $\bmod 2$ spectral sequence for $\mathbb{R}$. Again this makes for two cases, which we again call the first and second case.

Both $i_{1}\left(\eta \mu_{8 k+1}\right)$ and $\eta^{2} \beta_{8 k+2}$ map to zero in $K_{*}(\mathbb{C} ; \mathbb{Z} / 2)$, so in both cases these classes map to zero in $H^{0}(\mathbb{Q} ; \mathbb{Z} / 2)$, and admit an image in $H^{2}(\mathbb{Q} ; \mathbb{Z} / 2)$.

Consider the following diagram, with $i=4 k+3$ or $i=4 k+2$. Each edge map is only partially defined, on the kernel of the natural map to $K_{2 i-2}(\mathbb{C} ; \mathbb{Z} / 2)$.

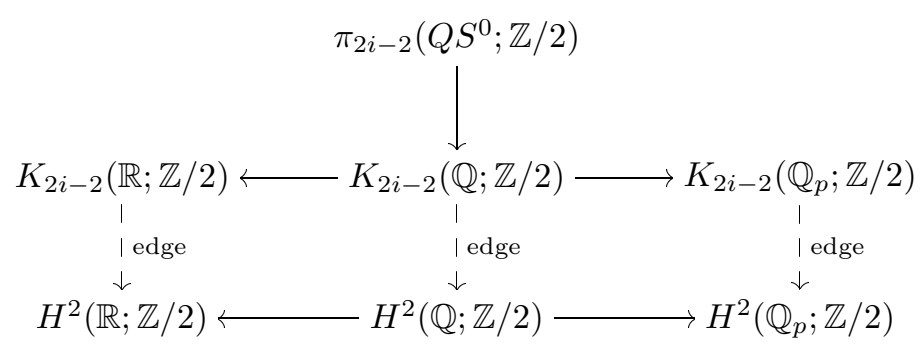

Define $x \in H^{2}(\mathbb{Q} ; \mathbb{Z} / 2), x_{p} \in H^{2}\left(\mathbb{Q}_{p} ; \mathbb{Z} / 2\right)$ and $z \in H^{2}(\mathbb{R} ; \mathbb{Z} / 2)$ as the images of $i_{1}\left(\eta \mu_{8 k+1}\right)$, resp. $\eta^{2} \beta_{8 k+2}$, as before. We need to prove that $z \neq 0$ in each case.

In both cases we know by Lemma 5.2 that $x_{2}$ is the nonzero element of the subgroup of $K_{2 i-2}\left(\mathbb{Q}_{2} ; \mathbb{Z} / 2\right)$ that maps isomorphically to $H^{2}\left(\mathbb{Q}_{2} ; \mathbb{Z} / 2\right)$ by the edge map. Thus also $x \neq 0$ in both cases.

For each prime $p \neq 2$ we have $x_{p}=0$. In the first case, this is because the image of $i_{1}\left(\eta \mu_{8 k+1}\right)$ in $K_{8 k+2}\left(\mathbb{Q}_{p} ; \mathbb{Z} / 2\right)$ factors through $K_{8 k+2}\left(\mathbb{Z}_{p} ; \mathbb{Z}_{2}\right) \cong K_{8 k+2}\left(\mathbb{F}_{p} ; \mathbb{Z}_{2}\right)=0$. In the second case, the image of $\eta^{2} \beta_{8 k+2}$ in $K_{8 k+4}\left(\mathbb{Q}_{p} ; \mathbb{Z} / 2\right)$ factors through

$$
K_{8 k+4}\left(\mathbb{Z}_{p} ; \mathbb{Z} / 2\right) \cong K_{8 k+4}\left(\mathbb{F}_{p} ; \mathbb{Z} / 2\right) \cong{ }_{2} K_{8 k+3}\left(\mathbb{F}_{p}\right),
$$

hence equals the image of $\eta^{2} \mu_{8 k+1} \in \pi_{8 k+3}\left(Q S^{0}\right)$ in $K_{8 k+3}\left(\mathbb{F}_{p}\right)$, which is zero. 
Again we have the exact localization sequence

$$
H^{2}\left(\mathbb{Z}\left[\frac{1}{2}\right] ; \mathbb{Z} / 2\right) \rightarrow H^{2}(\mathbb{Q} ; \mathbb{Z} / 2) \stackrel{\partial^{e}}{\longrightarrow} \bigoplus_{p \neq 2} H^{1}\left(\mathbb{F}_{p} ; \mathbb{Z} / 2\right)
$$

where $\partial^{e}$ takes $x$ to the sum over $p \neq 2$ of $\partial_{p}^{e}\left(x_{p}\right)=0$. Hence $x \neq 0$ admits a lift $y \neq 0 \in H^{2}\left(\mathbb{Z}\left[\frac{1}{2}\right] ; \mathbb{Z} / 2\right)$ in both cases. But then $z \neq 0$, because by Example 2.8 the natural map $H^{2}\left(\mathbb{Z}\left[\frac{1}{2}\right] ; \mathbb{Z} / 2\right) \rightarrow H^{2}(\mathbb{R} ; \mathbb{Z} / 2)$ is an isomorphism. This concludes the proof of Theorem 5.3 for $i_{1}\left(\eta \mu_{8 k+1}\right)$ and $\eta^{2} \beta_{8 k+2}$.

We now finish the proof of Theorem 5.3 by showing that the remaining classes do not survive to $E_{\infty}$. By [S1], the spectral sequence converges to

$$
K_{n}(\mathbb{R} ; \mathbb{Z} / 2) \cong \begin{cases}\mathbb{Z} / 2 & \text { for } n \equiv 0,1,3,4 \bmod 8 \\ \mathbb{Z} / 4 & \text { for } n \equiv 2 \bmod 8 \\ 0 & \text { otherwise. }\end{cases}
$$

We have shown that the classes $\beta_{8 k}, i_{1}\left(\mu_{8 k+1}\right), i_{1}\left(\eta \mu_{8 k+1}\right), \beta_{8 k+2}, \eta \beta_{8 k+2}$ and $\eta^{2} \beta_{8 k+2}$ survive as permanent cycles in this spectral sequence. They clearly generate the entire abutment, hence there can be no further permanent cycles in this spectral sequence, and the remaining classes must be killed by differentials. The classes in bidegrees $(p, q)$ with $p \equiv-2,-3 \bmod 4$ and $0 \leq p-q \leq 4$ cannot be hit by differentials for bidegree reasons, hence they must support nontrivial differentials. This completes the proof.

The mod $2^{\infty}$ case now follows by naturality.

Theorem 5.6. In the mod $2^{\infty}$ Bloch-Lichtenbaum spectral sequence for $\mathbb{R}$, the classes $\iota_{1}\left(\beta_{8 k+2}\right), \iota_{1}\left(\eta \beta_{8 k+2}\right)$ and $\iota_{1}\left(\eta^{2} \beta_{8 k+2}\right)$ in $\pi_{*}\left(Q S^{0} ; \mathbb{Z} / 2^{\infty}\right)$ map to classes in $K_{*}\left(\mathbb{R} ; \mathbb{Z} / 2^{\infty}\right)$ that are represented by permanent cycles in bidegrees $(p, q)$ with $p=-4 k-1$ and $0 \leq p-q \leq 2$. The classes $\mathbb{Z} / 2^{\infty}$ in bidegrees $(p, p)$ with $p=-4 k$ or $-4 k-2$ are also permanent cycles.

The remaining classes do not survive to the $E_{\infty}$-term. In particular, the classes in bidegrees $(p, q)$ with $p=-4 k-3$ and $0 \leq p-q \leq 5$ support nontrivial differentials.

There is a nontrivial additive extension $\mathbb{Z} / 2 \rtimes \mathbb{Z} / 2^{\infty} \cong \mathbb{Z} / 2^{\infty}$ in total degree $-(8 k+4)$.

Proof. Each permanent cycle of the mod 2 Bloch-Lichtenbaum spectral sequence for $\mathbb{R}$ maps under $\iota_{1}$ to an infinite cycle in the mod $2^{\infty}$ Bloch-Lichtenbaum spectral sequence for $\mathbb{R}$. Hence the classes $\beta_{8 k+2}, \eta \beta_{8 k+2}$ and $\eta^{2} \beta_{8 k+2}$ map to (nonzero) infinite cycles. They cannot be boundaries for bidegree reasons, hence are in fact permanent cycles. The natural map $K_{n}\left(\mathbb{R} ; \mathbb{Z} / 2^{\infty}\right) \rightarrow K_{n}\left(\mathbb{C} ; \mathbb{Z} / 2^{\infty}\right)$ is an isomorphism for $n=8 k$ and a surjection with kernel $\mathbb{Z} / 2$ when $n=8 k+4$. Hence the classes in bidegrees $(-4 k,-4 k)$ and $(-4 k-2,-4 k-2)$, which detect $K_{n}\left(\mathbb{C} ; \mathbb{Z} / 2^{\infty}\right)$ for $n=8 k$ and $n=8 k+4$, must also detect the image of $K_{n}\left(\mathbb{R} ; \mathbb{Z} / 2^{\infty}\right)$ in these degrees. Thus these classes are permanent cycles.

By Suslin's theorem [S1]

$$
K_{n}\left(\mathbb{R} ; \mathbb{Z} / 2^{\infty}\right) \cong \begin{cases}\mathbb{Z} / 2^{\infty} & \text { for } n \equiv 0,4 \bmod 8 \\ \mathbb{Z} / 2 & \text { for } n \equiv 2,3 \bmod 8 \\ 0 & \text { otherwise. }\end{cases}
$$

The given permanent cycles account for the entire abutment, and no other classes can survive to the $E_{\infty}$-term in this spectral sequence. For bidegree reasons the 
classes in bidegrees $(p, q)$ with $p \equiv-3 \bmod 4$ and $0 \leq p-q \leq 5$ cannot be hit by differentials, so the remaining classes in these bidegrees must support nontrivial differentials. This completes the proof.

\section{TWO-PRIMARY $K$-THEORY OF NUMBER FIELDS}

For number fields the $K$-theory localization sequence specializes as follows.

Theorem 6.1 (Soulé). Let $F$ be a number field with ring of $\ell$-integers $R$ and residue fields $k_{\mathfrak{P}}$ for $\mathfrak{P} \nmid \ell$. For $i \geq 1$ the $K$-theory localization sequence for $R$ breaks up into short exact sequences

$$
0 \rightarrow K_{2 i-1}\left(R ; \mathbb{Z} / 2^{\infty}\right) \rightarrow K_{2 i-1}\left(F ; \mathbb{Z} / 2^{\infty}\right) \stackrel{\partial}{\rightarrow} \bigoplus_{\mathfrak{P} \nmid 2} K_{2 i-2}\left(k_{\mathfrak{P}} ; \mathbb{Z} / 2^{\infty}\right) \rightarrow 0
$$

and isomorphisms $K_{2 i-2}\left(R ; \mathbb{Z} / 2^{\infty}\right) \cong K_{2 i-2}\left(F ; \mathbb{Z} / 2^{\infty}\right)$.

Proof. The terms $K_{n}\left(k_{\mathfrak{P}} ; \mathbb{Z} / 2^{\infty}\right)$ vanish for odd $n$ by Proposition 1.10. Soulé showed that the torsion group $K_{2 i-2}(F)$ maps onto $\bigoplus_{\mathfrak{P} \nmid 2} K_{2 i-3}\left(k_{\mathfrak{P}}\right)$; see [W1, 4.6]. It follows that in the commutative diagram

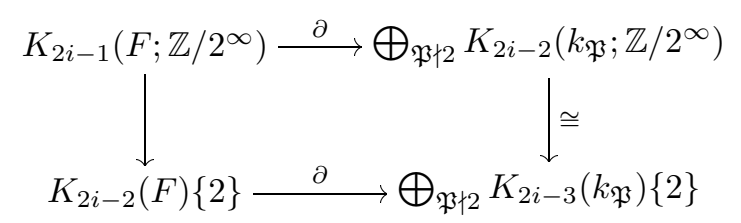

the left hand and lower maps are surjections, while the right hand map is an isomorphism. Hence the boundary map

$$
\partial: K_{2 i-1}\left(F ; \mathbb{Z} / 2^{\infty}\right) \rightarrow \bigoplus_{\mathfrak{P} \nmid 2} K_{2 i-2}\left(k_{\mathfrak{P}} ; \mathbb{Z} / 2^{\infty}\right)
$$

is surjective, as claimed.

The $K$-groups of rings of integers in number fields are known modulo torsion. The following result is proven in [Bo] and [Q3].

Theorem 6.2 (Borel, Quillen). Let $F$ be a number field with $r_{1}$ real embeddings, $r_{2}$ pairs of complex embeddings, and $s$ primes dividing 2 . For each $n \geq 0$ the group $K_{n}(R)$ is finitely generated, with rational rank

$$
\operatorname{dim}_{\mathbb{Q}}\left(K_{n}(R) \otimes_{\mathbb{Z}} \mathbb{Q}\right)= \begin{cases}1 & \text { for } n=0, \\ r_{1}+r_{2}+s-1 & \text { for } n=1, \\ r_{2} & \text { for } n \equiv 3 \bmod 4, \\ r_{1}+r_{2} & \text { for } n \equiv 1 \bmod 4, n>1, \\ 0 & \text { otherwise. }\end{cases}
$$

Likewise, the group $K_{n}\left(R ; \mathbb{Z} / \ell^{\infty}\right)$ is a discrete torsion group, Pontryagin dual to a finitely generated $\mathbb{Z}_{\ell}$-module of $\ell$-adic rank equal to the rational rank of $K_{n}(R)$.

For the remainder of this section we assume $\ell=2$.

We first dispense with the case when $F$ is totally imaginary. In this case, Theorem 1.13 expresses the $K$-groups of $F$ in terms of étale cohomology groups. We now use the localization sequences in $K$-theory and étale cohomology to express the $K$-groups of the ring of integers in $F$ in terms of étale cohomology. 
Theorem 6.3. Let $F$ be a totally imaginary number field, with $R$ its ring of 2 integers. The $\bmod 2^{\infty}$ algebraic $K$-groups of $R$ are given as follows:

$$
K_{n}\left(R ; \mathbb{Z} / 2^{\infty}\right) \cong \begin{cases}H^{0}(R ; W(i)) & \text { for } n=2 i \text { even }, \\ H^{1}(R ; W(i)) & \text { for } n=2 i-1 \text { odd }\end{cases}
$$

Proof. If $n=2 i$ we combine Theorem 1.13, Proposition 2.9(b) and Theorem 6.1 to get $K_{2 i}\left(R ; \mathbb{Z} / 2^{\infty}\right) \cong K_{2 i}\left(F ; \mathbb{Z} / 2^{\infty}\right) \cong H^{0}(F ; W(i)) \cong H^{0}(R ; W(i))$ for $i \geq 0$.

Now consider $n=2 i-1$ odd, with $i \geq 1$. By Theorems 1.12 and 1.13 the BlochLichtenbaum spectral sequences for $F$ and its completions $F_{\mathfrak{P}}$ determine isomorphisms $K_{2 i-1}\left(F ; \mathbb{Z} / 2^{\infty}\right) \cong H^{1}(F ; W(i))$ and $K_{2 i-1}\left(F_{\mathfrak{P}} ; \mathbb{Z} / 2^{\infty}\right) \cong H^{1}\left(F_{\mathfrak{P}} ; W(i)\right)$. By naturality of the spectral sequences with respect to the embeddings $F \rightarrow F_{\mathfrak{P}}$ we obtain the left commuting square in the following diagram:

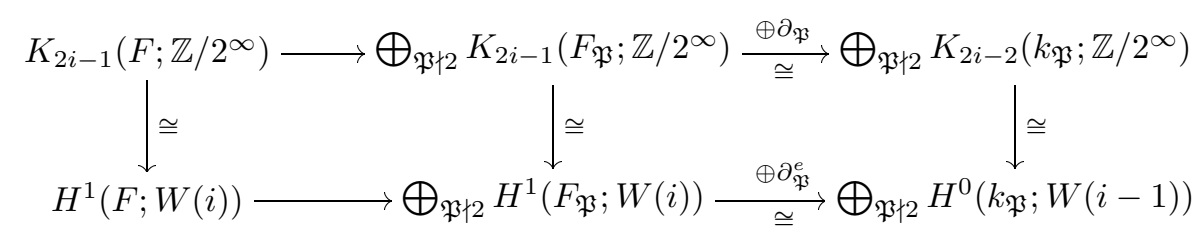

Now each map $\partial_{\mathfrak{P}}$ and $\partial_{\mathfrak{P}}^{e}$ is an isomorphism, by Corollaries 3.3 and 2.10 , respectively. Hence there is a unique isomorphism $K_{2 i-2}\left(k_{\mathfrak{P}} ; \mathbb{Z} / 2^{\infty}\right) \cong H^{0}\left(k_{\mathfrak{P}} ; W(i-1)\right)$ for each $\mathfrak{P} \nmid 2$ such that their direct sum makes the right square commute in the diagram above. The horizontal composites are $\partial$ and $\partial^{e}$, by naturality of the localization sequences with respect to the inclusions $R \rightarrow \mathcal{O}_{F_{\mathfrak{P}}}$.

The outer rectangle in the commutative diagram (6.4) induces maps in the middle and right positions between the short exact sequences of Theorem 6.1 and Proposition 4.7:

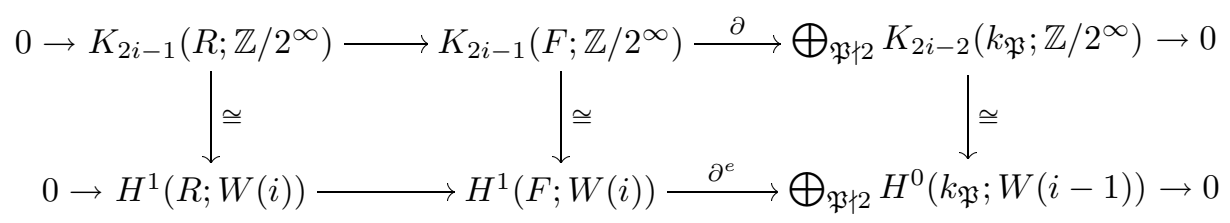

The left vertical map is the induced map from $\operatorname{ker}(\partial)$ to $\operatorname{ker}\left(\partial^{e}\right)$, and must be an isomorphism since the middle and right vertical maps are isomorphisms. Hence $K_{2 i-1}\left(R ; \mathbb{Z} / 2^{\infty}\right) \cong H^{1}(R ; W(i))$. This completes the proof.

We will shortly use the following algebraic lemma about comparing spectral sequences.

Lemma 6.5. Let $\alpha_{r}^{p, q}: E_{r}^{p, q} \rightarrow{ }^{\prime} E_{r}^{p, q}$ be a morphism of $E_{2}$-spectral sequences, both of which are zero for $p<q$. Suppose that: (i) all differentials in 'E are either zero or isomorphisms; (ii) the maps $\alpha_{2}^{p, q}$ are injections for $p=q$, surjections for $p=q+1$ and for $p=q+2$, and isomorphisms otherwise. Then:

(a) If $E_{\infty}^{p, q}={ }^{\prime} E_{2}^{p, q}$, then $E_{\infty}^{p, q}=E_{2}^{p, q}$.

(b) If ${ }^{\prime} E_{\infty}^{q, q}=0$ but $E_{2}^{q, q} \neq 0$, there is a unique $a=a(q), s=q+a$ and $t=q-a+1$ such that ${ }^{\prime} d_{a}:{ }^{\prime} E_{a}^{q, q} \cong{ }^{\prime} E_{a}^{s, t}$. Then $E_{\infty}^{q, q}=0$ and $E_{\infty}^{s, t} \cong{ }^{\prime} E_{2}^{q, q} / E_{2}^{q, q}$. 
(c) If $E_{\infty}^{q+1, q}=0$ but ${ }^{\prime} E_{2}^{q+1, q} \neq 0$, then $E_{\infty}^{q+1, q} \cong \operatorname{ker}\left(E_{2}^{q+1, q} \rightarrow{ }^{\prime} E_{2}^{q+1, q}\right)$.

(d) If $E_{\infty}^{q+2, q}=0$ but ${ }^{\prime} E_{2}^{q+2, q} \neq 0$, then $E_{\infty}^{q+2, q} \cong \operatorname{ker}\left(E_{2}^{q+2, q} \rightarrow{ }^{\prime} E_{2}^{q+2, q}\right)$.

(e) $E_{\infty}^{p, q} \cong{ }^{\prime} E_{\infty}^{p, q}$ in all other cases.

More specifically, let us call $(s, t)$ distinguished if there is a nonzero differential ${ }^{\prime} d_{a}:{ }^{\prime} E_{a}^{q, q} \cong{ }^{\prime} E_{a}^{s, t}$. Then $E_{r}^{s, t} \cong{ }^{\prime} E_{r}^{s, t}$ unless either $0 \leq t-s \leq 2$ or $(s, t)$ is distinguished and $r>a$. (These cases are implicitly itemized in (a) to (d) above.)

Proof. We proceed by induction on $r \geq 2$. Given $p, q$ and $r$ with $p \geq q$, set $s=p+r$ and $t=q-r+1$ and consider the following diagram:

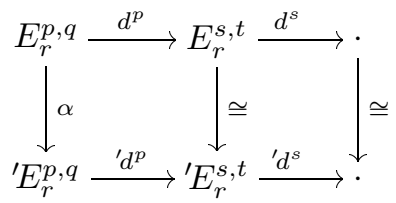

Here we briefly write $d^{p}$ for $d_{r}^{p, q}$, etc. Since $s \geq t+3$, the induction hypothesis implies that the right two vertical maps are isomorphisms.

If ' $d^{p}=0$, then $d^{p}=0$ and $E_{r+1}^{s, t} \cong{ }^{\prime} E_{r+1}^{s, t}$. Moreover, if $p<q+2 r-1$, then $' d^{p-r}=d^{p-r}=0$ and we also have $E_{r}^{p, q}=E_{r+1}^{p, q},{ }^{\prime} E_{r}^{p, q}={ }^{\prime} E_{r+1}^{p, q}$. If $p \geq q+2 r-1$, then $(p, q)$ is the $(s, t)$ of another such diagram.

Thus we may suppose that ${ }^{\prime} d^{p} \neq 0$. In this case $d^{s}={ }^{\prime} d^{s}=0,{ }^{\prime} E_{r+1}^{p, q}={ }^{\prime} E_{r+1}^{s, t}=0$, and $(p, q)$ cannot be distinguished. Thus we have only four cases. If $\alpha$ is an isomorphism, then $E_{r+1}^{p, q}=E_{r+1}^{s, t}=0$. If $p=q$, then $r=a(q)$ and $\alpha$ is an injection, so we have $E_{r+1}^{q, q}=0$ and $E_{r+1}^{s, t} \cong{ }^{\prime} E_{r}^{q, q} / E_{r}^{q, q}={ }^{\prime} E_{2}^{q, q} / E_{2}^{q, q}$. If $p=q+1$ or $p=q+2$, then $\alpha$ is a surjection, $E_{r+1}^{s, t}=0$ and $E_{r+1}^{p, q}=\operatorname{ker}(\alpha)$. This establishes the inductive step, and hence the lemma.

Definition 6.6 $\left(\tilde{H}^{n}\right.$ and $\left.\bar{H}^{n}\right)$. Suppose $F$ is a number field with $r_{1}>0$ real embeddings. Recall from 4.1 that $\alpha^{n}=\alpha^{n}(F ; M)$ denotes the natural map

$$
H^{n}(F ; M) \stackrel{\alpha^{n}}{\longrightarrow} \bigoplus^{r_{1}} H^{n}(\mathbb{R} ; M)
$$

where the sum runs over the real places of $F$. Let $\tilde{H}^{n}(F ; M)=\operatorname{ker} \alpha^{n}(F ; M)$.

For $n \geq 3$ the map $\alpha^{n}$ is an isomorphism by Tate's Theorem 4.2. Let $\bar{H}^{n}(F ; M)$ be the cokernel of the diagonal embedding

$$
\Delta: H^{n}(\mathbb{R} ; M) \rightarrow \bigoplus^{r_{1}} H^{n}(\mathbb{R} ; M) \cong H^{n}(F ; M)
$$

viewed as a quotient of $H^{n}(F ; M)$. When $M=W(i)$ and $i-n$ is odd the source and target of $\Delta$ are $\mathbb{Z} / 2$ and $(\mathbb{Z} / 2)^{r_{1}}$, so abstractly

$$
\bar{H}^{n}(F ; W(i))=\operatorname{cok} \Delta \cong(\mathbb{Z} / 2)^{r_{1}-1} .
$$

We use similar notations $\tilde{H}^{n}(R ; M)$ and $\bar{H}^{n}(R ; M)$ with $R$ replacing $F$. Clearly, $H^{n}(R ; M) \rightarrow H^{n}(F ; M)$ induces a natural map $\tilde{H}^{n}(R ; M) \rightarrow \tilde{H}^{n}(F ; M)$, and (for $n \geq 3)$ isomorphisms $\bar{H}^{n}(R ; M) \cong \bar{H}^{n}(F ; M)$. 
Theorem 6.7. Let $F$ be a real number field. Its $\bmod 2^{\infty}$ algebraic $K$-groups are given (up to extensions) as follows:

$$
K_{n}\left(F ; \mathbb{Z} / 2^{\infty}\right) \cong \begin{cases}H^{0}(F ; W(4 k)) \cong \mathbb{Z} / w_{4 k}(F) & \text { for } n=8 k, \\ H^{1}(F ; W(4 k+1)) & \text { for } n=8 k+1, \\ H^{0}(F ; W(4 k+1)) \cong \mathbb{Z} / 2 & \text { for } n=8 k+2, \\ H^{1}(F ; W(4 k+2)) & \text { for } n=8 k+3, \\ (\mathbb{Z} / 2)^{r_{1}} \rtimes H^{0}(F ; W(4 k+2)) & \text { for } n=8 k+4, \\ (\mathbb{Z} / 2)^{r_{1}-1} \rtimes H^{1}(F ; W(4 k+3)) & \text { for } n=8 k+5, \\ 0 & \text { for } n=8 k+6, \\ \tilde{H}^{1}(F ; W(4 k+4)) & \text { for } n=8 k+7 .\end{cases}
$$

Here $H^{0}(F ; W(4 k+2)) \cong \mathbb{Z} / w_{4 k+2}(F)$. The extension in degree $8 k+4$ is abstractly isomorphic to $(\mathbb{Z} / 2)^{r_{1}-1} \oplus \mathbb{Z} / 2 w_{4 k+2}(F)$. For any real embedding $F \rightarrow \mathbb{R}$ the image of $K_{8 k+4}\left(F ; \mathbb{Z} / 2^{\infty}\right)$ in $K_{8 k+4}\left(\mathbb{R} ; \mathbb{Z} / 2^{\infty}\right) \cong \mathbb{Z} / 2^{\infty}$ is the cyclic group $\mathbb{Z} / 2 w_{4 k+2}(F)$.

Proof. The mod $2^{\infty}$ Bloch-Lichtenbaum spectral sequence $E$ of (1.3) has

$$
E_{2}^{p, q}= \begin{cases}H^{p-q}(F ; W(-q)) & \text { for } q \leq p \leq 0 \\ 0 & \text { otherwise }\end{cases}
$$

Let ${ }^{\prime} E$ denote the direct sum of $r_{1}$ copies of the corresponding spectral sequence (1.3) for $\mathbb{R}$. We will apply Lemma 6.5 to the map $\alpha: E \rightarrow{ }^{\prime} E$ of spectral sequences induced by the $r_{1}$ real embeddings of $F$. The following lemma verifies that the hypotheses of 6.5 hold.

Lemma 6.8. Let $F$ be a real number field, and write $n=p-q$ and $i=-q$.

(a) For $n=0$

$$
\alpha^{0}: H^{0}(F ; W(i)) \rightarrow \bigoplus^{r_{1}} H^{0}(\mathbb{R} ; W(i))
$$

can be identified with the diagonal embeddings $\Delta: \mathbb{Z} / 2 \rightarrow(\mathbb{Z} / 2)^{r_{1}}$ for $i$ odd, and $\mathbb{Z} / w_{i}(F) \rightarrow\left(\mathbb{Z} / 2^{\infty}\right)^{r_{1}}$ for $i$ even.

(b) For $n=1$ we have a surjection

$$
\alpha^{1}: H^{1}(F ; W(i)) \rightarrow \bigoplus^{r_{1}} H^{1}(\mathbb{R} ; W(i))= \begin{cases}(\mathbb{Z} / 2)^{r_{1}} & i \text { even }, \\ 0 & i \text { odd } .\end{cases}
$$

(c) For $n=2$ and $i \geq 2$, or for $n \geq 3$, we have an isomorphism

$$
\alpha^{n}: H^{n}(F ; W(i)) \stackrel{\cong}{\longrightarrow} \bigoplus^{r_{1}} H^{n}(\mathbb{R} ; W(i))= \begin{cases}(\mathbb{Z} / 2)^{r_{1}} & i-n \text { even }, \\ 0 & i-n \text { odd } .\end{cases}
$$

Proof. To prove (a) it suffices to note that $H^{0}(F ; W(i)) \cong \mathbb{Z} / 2$ for $F$ real and $i$ odd, and that each real embedding induces an injection $H^{0}(F ; W(i)) \rightarrow H^{0}(\mathbb{R} ; W(i))$.

We turn to the proof of (b), where we may assume that $i$ is even. The coefficient inclusion $\iota_{1}: \mathbb{Z} / 2 \rightarrow W(i)$ induces the following commutative diagram:

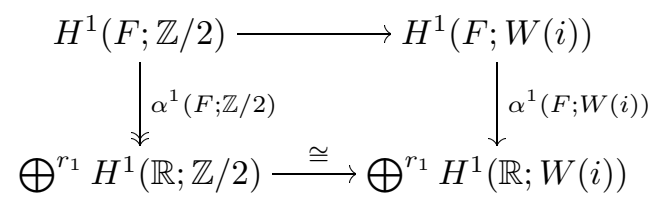


Note that $H^{1}(F ; \mathbb{Z} / 2) \cong F^{\times} / 2$. The left map $\alpha^{1}(F ; \mathbb{Z} / 2)$, which may be identified with $F^{\times} / 2 \rightarrow \bigoplus^{r_{1}} \mathbb{R}^{\times} / 2$, is surjective by the (very strong) approximation theorem for units. (See e.g. [CF, II.15]. The image of $F$ in $\mathbb{R}^{r_{1}}$ under the various real embeddings is dense.) Hence also $\alpha^{1}(F ; W(i))$ is surjective for all even $i$. This proves (b).

Claim (c) is immediate from Theorem 4.2 and Proposition 4.6.

We can now complete the proof of Theorem 6.7. By Theorem 5.6 and Lemma 6.5 the $E_{\infty}$-term of the mod $2^{\infty}$ Bloch-Lichtenbaum spectral sequence for $F$ is given as

$$
E_{\infty}^{p, q}= \begin{cases}H^{0}(F ; W(-q)) & \text { for } p=q,-p \not \equiv 3 \bmod 4, \\ H^{1}(F ; W(-q)) & \text { for } p=q+1,-p \neq 3 \bmod 4, \\ \tilde{H}^{1}(F ; W(-q)) & \text { for } p=q+1,-p \equiv 3 \bmod 4, \\ H^{2}(F ; W(-q)) & \text { for } p=q+2,-p \equiv 1 \bmod 4, \\ \bar{H}^{2 a-1}(F ; W(-q)) & \text { for }(p, q) \text { distinguished and } a \geq 2, \\ 0 & \text { otherwise. }\end{cases}
$$

There is precisely one distinguished bidegree $(p, q)$ in each total degree $p+q=$ $-(8 k+5)$, namely the bidegree hit by a nontrivial differential $d_{a}$ from bidegree $(-4 k-3,-4 k-3)$ in the spectral sequence for $\mathbb{R}$. (Here $a \geq 2$ might depend on $k$.) Then $p-q=n=2 a-1 \geq 3$, and $p$ is odd, so $q$ is even and $\bar{H}^{2 a-1}(F ; W(-q)) \cong$ $(\mathbb{Z} / 2)^{r_{1}-1}$. We also substitute $(\mathbb{Z} / 2)^{r_{1}}$ for $H^{2}(F ; W(4 k+3))$. The given formulas for $K_{*}\left(F ; \mathbb{Z} / 2^{\infty}\right)$ follow.

For the final assertion, set $i=4 k+2$ and consider the map of extensions from

$$
K_{8 k+4}\left(F ; \mathbb{Z} / 2^{\infty}\right) \cong H^{2}(F ; W(i+1)) \rtimes H^{0}(F ; W(i))
$$

to

$$
K_{8 k+4}\left(\mathbb{R} ; \mathbb{Z} / 2^{\infty}\right) \cong H^{2}(\mathbb{R} ; W(i+1)) \rtimes H^{0}(\mathbb{R} ; W(i)) \cong \mathbb{Z} / 2 \rtimes \mathbb{Z} / 2^{\infty}
$$

induced by a real embedding $F \rightarrow \mathbb{R}$. As noted in Theorem 5.6 , the second extension is nontrivial. Since the map from $\mathbb{Z} / w_{i}(F) \cong H^{0}(F ; W(i))$ to $H^{0}(\mathbb{R} ; W(i)) \cong$ $\mathbb{Z} / 2^{\infty}$ is injective, the first extension $K_{8 k+4}\left(F ; \mathbb{Z} / 2^{\infty}\right)$ must be nontrivial, with image $\mathbb{Z} / 2 w_{i}(F)$. Finally, note that all nontrivial extensions of $\mathbb{Z} / w_{i}$ by $(\mathbb{Z} / 2)^{r_{1}}$ are abstractly isomorphic.

Theorem 6.9. Let $F$ be a real number field, with $R$ its ring of 2-integers. The mod $2^{\infty}$ algebraic $K$-groups of $R$ are given as follows:

$$
K_{n}\left(R ; \mathbb{Z} / 2^{\infty}\right) \cong \begin{cases}H^{0}(R ; W(4 k)) \cong \mathbb{Z} / w_{4 k}(F) & \text { for } n=8 k, \\ H^{1}(R ; W(4 k+1)) & \text { for } n=8 k+1, \\ H^{0}(R ; W(4 k+1)) \cong \mathbb{Z} / 2 & \text { for } n=8 k+2, \\ H^{1}(R ; W(4 k+2)) & \text { for } n=8 k+3, \\ (\mathbb{Z} / 2)^{r_{1}} \rtimes H^{0}(R ; W(4 k+2)) & \text { for } n=8 k+4, \\ (\mathbb{Z} / 2)^{r_{1}-1} \rtimes H^{1}(R ; W(4 k+3)) & \text { for } n=8 k+5, \\ 0 & \text { for } n=8 k+6, \\ \tilde{H}^{1}(R ; W(4 k+4)) & \text { for } n=8 k+7 .\end{cases}
$$

Here $H^{0}(R ; W(4 k+2)) \cong \mathbb{Z} / w_{4 k+2}(F)$. The extension in degree $8 k+4$ is abstractly isomorphic to $(\mathbb{Z} / 2)^{r_{1}-1} \oplus \mathbb{Z} / 2 w_{4 k+2}(F)$. 
Proof. The result is immediate from Theorem 6.7 when $n=2 i$, because we have $K_{2 i}\left(R ; \mathbb{Z} / 2^{\infty}\right) \cong K_{2 i}\left(F ; \mathbb{Z} / 2^{\infty}\right)$ by Theorem 6.1 and $H^{0}(R ; W(i))=H^{0}(F ; W(i))$.

When $n=2 i-1$ we compare the edge maps $K_{2 i-1}\left(F ; \mathbb{Z} / 2^{\infty}\right) \rightarrow H^{1}(F ; W(i))$ for $i \geq 1$ with the edge isomorphisms

$$
K_{2 i-1}\left(F_{\mathfrak{P}} ; \mathbb{Z} / 2^{\infty}\right) \stackrel{\cong}{\longrightarrow} H^{1}\left(F_{\mathfrak{P}} ; W(i)\right)
$$

for each $\mathfrak{P} \nmid 2$. As in the construction of diagram (6.4), the isomorphisms $\partial_{\mathfrak{P}}$ and $\partial_{\mathfrak{P}}^{e}$ determine isomorphisms $K_{2 i-2}\left(k_{\mathfrak{P}} ; \mathbb{Z} / 2^{\infty}\right) \rightarrow H^{0}\left(k_{\mathfrak{P}} ; W(i-1)\right)$ making the right square in the following diagram commute:

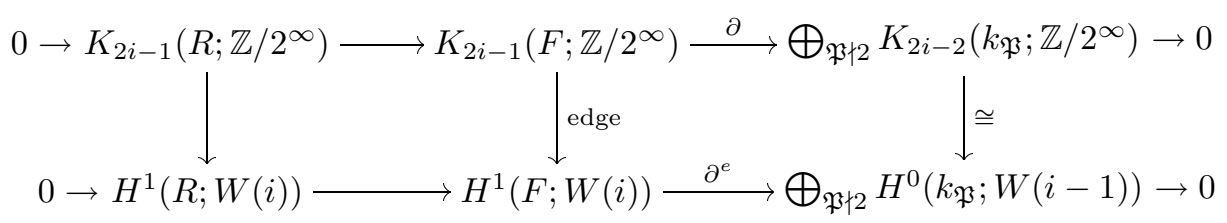

The rows are exact by Proposition 4.7 and Theorem 6.1.

In degrees $n=8 k+1$ and $8 k+3$ the edge map for $F$ in (6.10) is an isomorphism by Theorem 6.7 . Hence the induced map $K_{2 i-1}\left(R ; \mathbb{Z} / 2^{\infty}\right) \rightarrow H^{1}(R ; W(i))$ is also an isomorphism, by the 5-lemma.

In degrees $n=8 k+5$ the edge map for $F$ in (6.10) is a surjection, with kernel $(\mathbb{Z} / 2)^{r_{1}-1}$. It follows that we have a short exact sequence

$$
0 \rightarrow(\mathbb{Z} / 2)^{r_{1}-1} \rightarrow K_{8 k+5}\left(R ; \mathbb{Z} / 2^{\infty}\right) \rightarrow H^{1}(R ; W(4 k+3)) \rightarrow 0
$$

where the right hand map is the map of horizontal kernels in (6.10).

In degrees $n=8 k+7$ the edge map for $F$ in (6.10) is injective, and its cokernel can be identified with $\bigoplus^{r_{1}} H^{1}(\mathbb{R} ; W(4 k+4))$ in view of Definition 6.6 and Lemma $6.8(\mathrm{~b})$. Hence by the snake lemma applied to (6.10) there is a short exact sequence

$$
0 \rightarrow K_{8 k+7}\left(R ; \mathbb{Z} / 2^{\infty}\right) \rightarrow H^{1}(R ; W(4 k+4)) \stackrel{\alpha^{1}}{\longrightarrow} \bigoplus^{r_{1}} H^{1}(\mathbb{R} ; W(4 k+4)) \rightarrow 0
$$

where the right hand map is the direct sum of the natural maps induced by the real embeddings $R \rightarrow F \rightarrow \mathbb{R}$. Hence $K_{8 k+7}\left(R ; \mathbb{Z} / 2^{\infty}\right) \cong \tilde{H}^{1}(R ; W(4 k+4))$.

Corollary 6.11. The natural maps

$$
\begin{aligned}
& \alpha^{1}: H^{1}(R ; W(i)) \rightarrow \bigoplus^{r_{1}} H^{1}(\mathbb{R} ; W(i)) \cong(\mathbb{Z} / 2)^{r_{1}}, \\
& \alpha^{2}: H^{2}\left(R ; \mathbb{Z}_{2}(i)\right) \rightarrow \bigoplus H^{2}\left(\mathbb{R} ; \mathbb{Z}_{2}(i)\right) \cong(\mathbb{Z} / 2)^{r_{1}}
\end{aligned}
$$

are surjective for $i=4 k>0$.

Proof. The first claim follows from the last paragraph of the proof above. The second claim follows by a Bockstein argument.

Hence we have formulas describing the 2-primary $K$-theory of $R$, and thus of $\mathcal{O}_{F}$, in terms of the étale cohomology of $R$. We recall that $H^{n}(R ; W(i))$ is completely described for $n=0$ by Propositions 1.8 and $2.9(\mathrm{~b})$, for $n=2$ and $i \geq 1$ by Propositions $2.7(\mathrm{~b})$ and 4.6 , and for $n \geq 3$ by Corollary 4.4. Hence only $H^{1}(R ; W(i))$ remains unknown; we shall see in Appendix A that when $F$ is totally real it is related to $\zeta_{F}(1-i)$ via the Main Conjecture of Iwasawa Theory. 
We now translate from $H^{n}(R ; W(i))$ with mod $2^{\infty}$-coefficients to $H^{n}\left(R ; \mathbb{Z}_{2}(i)\right)$ with 2-adic coefficients, using the universal coefficient theorem 2.4.

Proposition 6.12. Let $R$ be the ring of 2-integers in a number field $F$. The group $H^{n}(R ; W(i))$ is the Pontryagin dual of a finitely generated $\mathbb{Z}_{2}$-module for all $n$ and $i$. Likewise $H^{n}\left(R ; \mathbb{Z}_{2}(i)\right)$ is a finitely generated $\mathbb{Z}_{2}$-module for all $n$ and $i$.

For $i \geq 2$ the groups $H^{0}(R ; W(i)) \cong \mathbb{Z} / w_{i}(F)$ and $H^{2}\left(R ; \mathbb{Z}_{2}(i)\right)$ are finite, and

$$
\begin{aligned}
& H^{1}\left(R ; \mathbb{Z}_{2}(i)\right) \cong\left(\mathbb{Z}_{2}\right)^{r} \oplus \mathbb{Z} / w_{i}(F), \\
& H^{1}(R ; W(i)) \cong\left(\mathbb{Z} / 2^{\infty}\right)^{r} \oplus H^{2}\left(R ; \mathbb{Z}_{2}(i)\right)
\end{aligned}
$$

where $r=r_{2}$ for $i$ even, and $r=r_{1}+r_{2}$ for $i$ odd.

The finite group $H^{2}\left(R ; \mathbb{Z}_{2}(i)\right)$ is determined up to extensions by the exact sequence

$$
\begin{aligned}
& 0 \rightarrow H^{0}(R ; W(1-i)) \stackrel{\beta^{0}}{\longrightarrow} \bigoplus_{v \in S_{\infty}} \hat{H}^{0}\left(F_{v} ; W(1-i)\right) \rightarrow \\
& \quad \rightarrow H^{2}\left(R ; \mathbb{Z}_{2}(i)\right) \rightarrow \amalg^{1}(R ; W(1-i)) \rightarrow 0 .
\end{aligned}
$$

In particular each Tate-Shafarevich group $\amalg^{1}(R ; W(1-i))$ is finite for $i \geq 2$.

Proof. Finite generation of $H^{n}(R ; W(i))^{\#}$ for $i>1$ is clear from Quillen's part of Theorem 6.2, the universal coefficient theorem, and Theorems 6.3 and 6.9. The corresponding statements for $H^{n}\left(R ; \mathbb{Z}_{2}(i)\right)$ follow by the universal coefficient theorem 2.4, and for $H^{n}(R ; W(1-i))^{\#}$ and $H^{n}\left(R ; \mathbb{Z}_{2}(1-i)\right)$ by global Tate duality, i.e., the exact sequence in Theorem 4.9(b). Similar remarks apply for $i=1$ by use of Proposition 2.7(b).

For $i \geq 1$ the rank of $H^{1}(R ; W(i))^{\#}$ modulo torsion as a free $\mathbb{Z}_{2}$-module equals the rational rank of $K_{2 i-1}(R)$, by Theorems 6.3 and 6.9 , and is given by Borel's part of Theorem 6.2. For $i \geq 2$ the same reasoning shows that $H^{2}(R ; W(i))$ is finite for $i \geq 2$. Thus $H^{2}\left(R ; \mathbb{Z}_{2}(i)\right)$ is isomorphic to the torsion in $H^{1}(R ; W(i))^{\#}$ by the universal coefficient theorem. By global Tate duality, this group is given by the 4-term exact sequence listed above, where $\amalg^{1}(R ; W(1-i))$ appears as the kernel of $\beta^{1}$ in Tate's 9-term exact sequence 4.9(b).

In particular $\amalg^{1}(R ; W(1-i))$ is a quotient of the finite group $H^{2}(R ; W(i))$ and is itself finite.

Proposition 6.13. Let $R$ be the ring of 2-integers in a number field $F$ with $r_{1}$ real embeddings and $r_{2}$ pairs of complex embeddings. Let $s$ be the number of primes of $F$ dividing 2, and let $t$ be the 2-rank of the Picard group $\operatorname{Pic}(R)$. Then for $i \geq 2$ the 2 -rank of the finite group $H^{2}\left(R ; \mathbb{Z}_{2}(i)\right)$ equals

$$
\begin{cases}r_{1}+s+t-1 & \text { for } i \text { even } \\ s+t-1 & \text { for } i \text { odd. }\end{cases}
$$

Proof. There is an exact universal coefficient sequence

$$
0 \rightarrow H^{2}\left(R ; \mathbb{Z}_{2}(i)\right) / 2 \rightarrow H^{2}(R ; \mathbb{Z} / 2) \rightarrow{ }_{2} H^{3}\left(R ; \mathbb{Z}_{2}(i)\right) \rightarrow 0 .
$$

By Corollary 4.4 and Propositions 2.4 and 4.6, if $i \geq 2$ then $H^{3}\left(R ; \mathbb{Z}_{2}(i)\right)$ is 0 for $i$ even and $(\mathbb{Z} / 2)^{r_{1}}$ for $i$ odd. Also $H^{2}(R ; \mathbb{Z} / 2) \cong \operatorname{Pic}(R) / 2 \oplus_{2} \operatorname{Br}(R)$ by the Kummer sequence. Now $\operatorname{Pic}(R) / 2$ has rank $t$ and ${ }_{2} \operatorname{Br}(R)$ has rank $r_{1}+s-1$. So $H^{2}(R ; \mathbb{Z} / 2)$ has rank $r_{1}+s+t-1$ and the result follows. 
We can now explicitly formulate a result about the 2-torsion in the algebraic $K$-groups of $R$. Recall from 6.6 and 6.11 that $\tilde{H}^{2}\left(R ; \mathbb{Z}_{2}(4 k+4)\right)$ denotes the kernel of the natural surjective map $\alpha^{2}$ from $H^{2}\left(R ; \mathbb{Z}_{2}(4 k+4)\right)$ to $(\mathbb{Z} / 2)^{r_{1}}$ induced by the $r_{1}$ real embeddings of $F$.

Theorem 6.14. (a) Let $F$ be a totally imaginary number field. The 2-torsion subgroup in the algebraic $K$-group $K_{n}(R)$ is given for $n \geq 0$ by

$$
K_{n}(R)\{2\} \cong \begin{cases}H^{2}\left(R ; \mathbb{Z}_{2}(i+1)\right) & \text { for } n=2 i \text { even } \\ \mathbb{Z} / w_{i}(F) & \text { for } n=2 i-1 \text { odd }\end{cases}
$$

The 2-primary Harris-Segal summand in $K_{2 i-1}(R)$ equals the 2-torsion subgroup $K_{2 i-1}(R)\{2\} \cong \mathbb{Z} / w_{i}(F)$, while the number of cyclic summands in the finite group $K_{2 i}(R)\{2\} \cong H^{2}\left(R ; \mathbb{Z}_{2}(i+1)\right)$ is $s+t-1$, independent of $i \geq 1$.

(b) Let $F$ be a real number field. The 2-torsion subgroup in the algebraic $K$-group $K_{n}(R)$ is given for $n \geq 0$ by

$$
K_{n}(R)\{2\} \cong \begin{cases}H^{2}\left(R ; \mathbb{Z}_{2}(4 k+1)\right) & \text { for } n=8 k, \\ \mathbb{Z} / 2 & \text { for } n=8 k+1, \\ H^{2}\left(R ; \mathbb{Z}_{2}(4 k+2)\right) & \text { for } n=8 k+2, \\ (\mathbb{Z} / 2)^{r_{1}-1} \oplus \mathbb{Z} / 2 w_{4 k+2}(F) & \text { for } n=8 k+3, \\ (\mathbb{Z} / 2)^{\rho} \rtimes H^{2}\left(R ; \mathbb{Z}_{2}(4 k+3)\right) & \text { for } n=8 k+4, \text { with } \rho<r_{1}, \\ 0 & \text { for } n=8 k+5, \\ \tilde{H}^{2}\left(R ; \mathbb{Z}_{2}(4 k+4)\right) & \text { for } n=8 k+6, \\ \mathbb{Z} / w_{4 k+4}(F) & \text { for } n=8 k+7 .\end{cases}
$$

The 2-primary Harris-Segal summands in $K_{8 k+3}(R)$ and $K_{8 k+7}(R)$ are the cyclic groups $\mathbb{Z} / 2 w_{4 k+2}$ and $\mathbb{Z} / w_{4 k+4}$, respectively.

The undetermined extension $K_{8 k+4}(R)\{2\}$ is isomorphic to the maximal finite quotient of $K_{8 k+5}\left(R ; \mathbb{Z} / 2^{\infty}\right)$. The latter $K$-group fits into the short exact sequence

$$
0 \rightarrow(\mathbb{Z} / 2)^{r_{1}-1} \rightarrow K_{8 k+5}\left(R ; \mathbb{Z} / 2^{\infty}\right) \rightarrow\left(\mathbb{Z} / 2^{\infty}\right)^{r_{1}+r_{2}} \oplus H^{2}\left(R ; \mathbb{Z}_{2}(4 k+3)\right) \rightarrow 0 .
$$

Part (a) of this theorem is restated as Theorem 0.4 in the introduction, and part (b) is restated as Theorem 0.6. Both of these results use Theorem 6.2. The orders of the Harris-Segal summands had previously been known up to a factor of 2 ; see [W1, 6.8.1]. See Corollary 7.12 for more information about the extensions in the above result.

Proof. Combine Theorems 6.3 and 6.9 with the universal coefficient theorem. Substitute $\mathbb{Z} / w_{i}(F)$ for $H^{0}(R ; W(i))$, and note that $w_{i}(F)=2$ for $i$ odd and $F$ real.

\section{Mod Two $K$-Groups OF NUMBER FIELDS}

In this section we use the mod 2 Bloch-Lichtenbaum spectral sequence (1.2) to compute the mod 2 algebraic $K$-theory of number fields and their rings of integers. Combined with the universal coefficient theorem we can thus compute the number of cyclic summands in the 2-torsion subgroups of $K$-theory. In particular we can detect nontrivial additive extensions in Theorems 6.9 and 6.14 . 
Proposition 7.1. The edge maps $K_{2 i-1}(F ; \mathbb{Z} / 2) \rightarrow H^{1}(F ; \mathbb{Z} / 2)$ in the $\bmod 2$ Bloch-Lichtenbaum spectral sequence induce maps $K_{2 i-1}(R ; \mathbb{Z} / 2) \rightarrow H^{1}(R ; \mathbb{Z} / 2)$ fitting into a commutative diagram with exact rows:

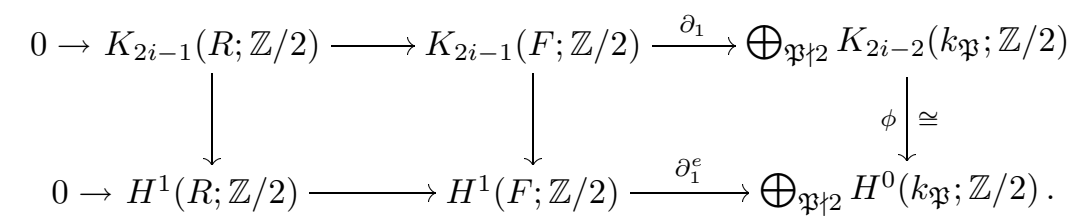

Proof. We have $K_{2 i-1}(R) \cong K_{2 i-1}(F)$ and $K_{2 i-2}(R)$ injects into $K_{2 i-2}(F)$; this is a theorem of Soulé (see $[\mathrm{W} 1,4.6])$. Hence $K_{2 i-1}(R ; \mathbb{Z} / 2)$ injects into $K_{2 i-1}(F ; \mathbb{Z} / 2)$ and the top row is exact. Now the construction of the right square proceeds exactly like the construction of the commutative square (6.4), using the identification of $K_{2 i-2}\left(k_{\mathfrak{P}} ; \mathbb{Z} / 2\right)$ and $H^{0}\left(k_{\mathfrak{P}} ; \mathbb{Z} / 2\right)$ with the exponent two subgroups of the cyclic groups $K_{2 i-2}\left(k_{\mathfrak{P}} ; \mathbb{Z} / 2^{\infty}\right)$ and $H^{0}\left(k_{\mathfrak{P}} ; W(i-1)\right)$.

Now let $F$ be a totally imaginary number field. The mod 2 algebraic $K$-groups of $F$ were given in Theorem 1.15(b); here are the mod $2 K$-groups of $R$. We remind the reader that $H^{0}(R ; \mathbb{Z} / 2)=\mathbb{Z} / 2, H^{1}(R ; \mathbb{Z} / 2) \cong R^{\times} / 2 \oplus{ }_{2} \operatorname{Pic}(R)$, and $H^{2}(R ; \mathbb{Z} / 2) \cong \operatorname{Pic}(R) / 2 \oplus_{2} \operatorname{Br}(R)$.

Theorem 7.2. Let $F$ be a totally imaginary number field, with $R$ its ring of 2integers. The mod 2 algebraic $K$-groups of $R$ are given (up to extensions) as follows:

$$
K_{n}(R ; \mathbb{Z} / 2) \cong \begin{cases}\operatorname{Pic}(R) / 2 \oplus H^{0}(R ; \mathbb{Z} / 2) & \text { for } n=0, \\ H^{1}(R ; \mathbb{Z} / 2) & \text { for } n=2 i-1 \text { odd }, \\ H^{2}(R ; \mathbb{Z} / 2) \rtimes H^{0}(R ; \mathbb{Z} / 2) & \text { for } n=2 i>0 \text { even. }\end{cases}
$$

The extension for $n=2 i$ need not split; see Remark 1.16(b).

Proof. The case $n=0$ follows trivially from $K_{0}(R) \cong \mathbb{Z} \oplus \operatorname{Pic}(R)$.

The case $n=2 i>0$ follows from Theorems 4.5(a) and 6.3, which imply the existence of isomorphisms

$$
H^{2}(R ; \mathbb{Z} / 2) \cong H^{1}(R ; W(i+1)) / 2 \cong K_{2 i+1}\left(R ; \mathbb{Z} / 2^{\infty}\right) / 2
$$

and $H^{0}(R ; \mathbb{Z} / 2) \cong{ }_{2} H^{0}(R ; W(i)) \cong{ }_{2} K_{2 i}\left(R ; \mathbb{Z} / 2^{\infty}\right)$. The claim then follows from the exact sequence

$$
0 \rightarrow K_{2 i+1}\left(R ; \mathbb{Z} / 2^{\infty}\right) / 2 \rightarrow K_{2 i}(R ; \mathbb{Z} / 2) \rightarrow{ }_{2} K_{2 i}\left(R ; \mathbb{Z} / 2^{\infty}\right) \rightarrow 0 .
$$

This leaves the odd cases $n=2 i-1$ to consider, $i \geq 1$. As in the proof of Theorem 6.3 the Bloch-Lichtenbaum spectral sequence determines an isomorphism $K_{2 i-1}(F ; \mathbb{Z} / 2) \cong H^{1}(F ; \mathbb{Z} / 2)$. Hence the theorem follows in this case by the 5 lemma applied to the diagram of Proposition 7.1.

We now describe the situation for real number fields.

Definition 7.3 $\left(\mathrm{Pic}_{+}\right.$and $\left.\mathrm{Br}_{+}\right)$. Let $F$ be a real number field. Each real embedding of $F$ determines a map $F^{\times} \rightarrow \mathbb{R}^{\times} \rightarrow \mathbb{R}^{\times} / 2 \cong \mathbb{Z} / 2$, detecting the sign of units of $F$ under that embedding. Let the sign map

$$
\sigma: F^{\times} \rightarrow(\mathbb{Z} / 2)^{r_{1}}
$$


be the direct sum of these maps. Clearly $\sigma$ factors over $F^{\times} / 2$, and we let $\sigma / 2$ denote the resulting map $F^{\times} / 2 \rightarrow(\mathbb{Z} / 2)^{r_{1}}$, which can be identified with $\alpha^{1}(F ; \mathbb{Z} / 2)$ from Definition 4.1 .

As noted in the proof of Lemma 6.8(b), the (very strong) approximation theorem for $F$ implies that the image of $\sigma$ is dense, hence that $\sigma$ is surjective. We let $F_{+}^{\times} \subset F^{\times}$be the kernel of $\sigma$. This is the group of totally positive units of $F$. Let $R_{+}^{\times}=R^{\times} \cap F_{+}^{\times}$be the subgroup of totally positive units in $R$. Let $e_{+}$be the restriction of the divisor map $e: F^{\times} \rightarrow \bigoplus_{\mathfrak{P} \nmid \ell} \mathbb{Z}$ to $F_{+}^{\times}$, and let $\operatorname{Pic}_{+}(R)=$ cok $e_{+}$ be the narrow Picard group, by definition. We have a diagram with exact rows:

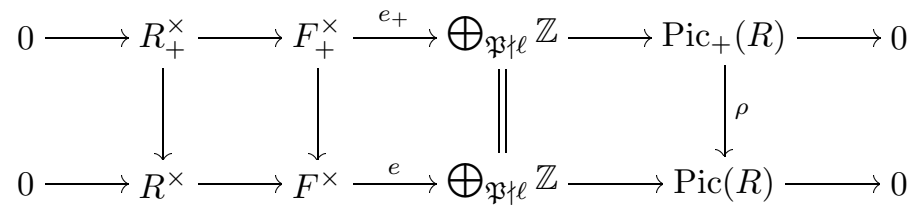

It follows that $\operatorname{Pic}_{+}(R)$ is an extension of the finite group $\operatorname{Pic}(R)$ by an elementary Abelian 2-group. Indeed, the snake lemma yields an exact sequence

$$
0 \rightarrow R_{+}^{\times} \rightarrow R^{\times} \rightarrow(\mathbb{Z} / 2)^{r_{1}} \rightarrow \operatorname{Pic}_{+}(R) \stackrel{\rho}{\rightarrow} \operatorname{Pic}(R) \rightarrow 0
$$

Let $t$ be the 2-rank of $\operatorname{Pic}(R)$, and let $u$ be the 2-rank of $\operatorname{Pic}_{+}(R)$. (Recall that the 2-rank of a group $A$ is the dimension of ${ }_{2} A$ over $\mathbb{F}_{2}$.) We call $j=u-t$ the signature defect of $R$. Clearly $t \leq u<t+r_{1}$ when $F$ is real, since $-1 \in R^{\times}$ maps nontrivially to $(\mathbb{Z} / 2)^{r_{1}}$. Thus $0 \leq j<r_{1}$ in the real case. We will see in Lemma 7.6 (a) that the 2-rank of $\tilde{H}^{1}(R ; \mathbb{Z} / 2)$ is $r_{2}+s+u$.

The real embeddings of $F$ determine surjective maps $\operatorname{Br}(F) \rightarrow \operatorname{Br}(\mathbb{R}) \cong \mathbb{Z} / 2$, which combine to a projection map $\tau: \operatorname{Br}(F) \rightarrow(\mathbb{Z} / 2)^{r_{1}}$. By the Brauer-HasseNoether theorem (2.6) the map $\tau$ is split surjective, since $F$ has at least one nonArchimedean place. Likewise the restriction ${ }_{2} \tau$ of $\tau$ to the elements of exponent 2 is a surjective $\operatorname{map}{ }_{2} \tau:{ }_{2} \operatorname{Br}(F) \rightarrow(\mathbb{Z} / 2)^{r_{1}}$, which can be identified with $\alpha^{2}(F ; \mathbb{Z} / 2)$. Let $\operatorname{Br}_{+}(F)=\operatorname{ker} \tau$, and use similar notation with $R$ replacing $F$. Note that $\mathrm{Br}_{+}(R) \cong(\mathbb{Q} / \mathbb{Z})^{s-1}$ by $(2.6)$. The kernel of ${ }_{2} \tau$ is $\tilde{H}^{2}(F ; \mathbb{Z} / 2) \cong{ }_{2} \mathrm{Br}_{+}(F)$.

Recall the groups $\tilde{H}^{n}(F ; \mathbb{Z} / 2)$ and $\bar{H}^{n}(F ; \mathbb{Z} / 2)$ introduced in Definition 6.6.

Theorem 7.4. Let $F$ be a real number field. Its mod 2 algebraic $K$-groups are given (up to extensions) for $n>0$ as follows:

$$
K_{n}(F ; \mathbb{Z} / 2) \cong \begin{cases}\tilde{H}^{2}(F ; \mathbb{Z} / 2) \rtimes H^{0}(F ; \mathbb{Z} / 2) & \text { for } n=8 k, \\ H^{1}(F ; \mathbb{Z} / 2) & \text { for } n=8 k+1, \\ H^{2}(F ; \mathbb{Z} / 2) \rtimes H^{0}(F ; \mathbb{Z} / 2) & \text { for } n=8 k+2, \\ (\mathbb{Z} / 2)^{r_{1}-1} \rtimes H^{1}(F ; \mathbb{Z} / 2) & \text { for } n=8 k+3, \\ H^{2}(F ; \mathbb{Z} / 2) & \text { for } n=8 k+4, \\ (\mathbb{Z} / 2)^{r_{1}-1} \rtimes \tilde{H}^{1}(F ; \mathbb{Z} / 2) & \text { for } n=8 k+5, \\ \tilde{H}^{2}(F ; \mathbb{Z} / 2) & \text { for } n=8 k+6, \\ \tilde{H}^{1}(F ; \mathbb{Z} / 2) & \text { for } n=8 k+7 .\end{cases}
$$


Proof. The mod 2 Bloch-Lichtenbaum spectral sequence converging to $K_{*}(F ; \mathbb{Z} / 2)$ has

$$
E_{2}^{p, q}= \begin{cases}H^{p-q}(F ; \mathbb{Z} / 2) & \text { for } q \leq p \leq 0 \\ 0 & \text { otherwise. }\end{cases}
$$

Here $H^{0}(F ; \mathbb{Z} / 2)=\mathbb{Z} / 2, H^{1}(F ; \mathbb{Z} / 2) \cong F^{\times} / 2, H^{2}(F ; \mathbb{Z} / 2) \cong{ }_{2} \operatorname{Br}(F)$. For $n \geq 3$ we have $H^{n}(F ; \mathbb{Z} / 2) \cong(\mathbb{Z} / 2)^{r_{1}}$. As in the proof of Theorem 6.7 we compare with the sum of $r_{1}$ copies of the spectral sequence for $\mathbb{R}$, using Lemma 6.5.

In the mod 2 case there are nontrivial $d_{a}$-differentials from the line $p=q$ in the spectral sequence for $\mathbb{R}$ precisely when $p=q \equiv-2,-3 \bmod 4$, and these hit one distinguished bidegree $(s, t)$ in each total degree $s+t=-(8 k+3)$ or $s+t=-(8 k+5)$. Then $a \geq 2$, so $2 a-1 \geq 3$.

Lemma 7.5. Let $F$ be a real number field and write $n=p-q$. Consider $\alpha^{n}=$ $\alpha^{n}(F ; \mathbb{Z} / 2)$.

(a) $\alpha^{0}$ is identified with the diagonal embedding $\Delta: \mathbb{Z} / 2 \rightarrow(\mathbb{Z} / 2)^{r_{1}}$.

(b) $\alpha^{1}$ is identified with the surjective sign map $\sigma / 2: F^{\times} / 2 \rightarrow(\mathbb{Z} / 2)^{r_{1}}$.

(c) $\alpha^{2}$ is identified with the surjective projection ${ }_{2} \tau:{ }_{2} \operatorname{Br}(F) \rightarrow(\mathbb{Z} / 2)^{r_{1}}$.

(d) For $n \geq 3, \alpha^{n}$ is an isomorphism.

Proof. The proof proceeds like the proof of Lemma 6.8 above.

Combining Theorem 5.3 and Lemma 6.5 with the lemma above, we are left with the following $E_{\infty}$-term:

$$
E_{\infty}^{p, q}= \begin{cases}H^{0}(F ; \mathbb{Z} / 2) & \text { for } p=q,-p \equiv 0,1 \bmod 4, \\ H^{1}(F ; \mathbb{Z} / 2) & \text { for } p=q+1,-p \equiv 0,1 \bmod 4, \\ \tilde{H}^{1}(F ; \mathbb{Z} / 2) & \text { for } p=q+1,-p \equiv 2,3 \bmod 4, \\ H^{2}(F ; \mathbb{Z} / 2) & \text { for } p=q+2,-p \equiv 0,1 \bmod 4, \\ \tilde{H}^{2}(F ; \mathbb{Z} / 2) & \text { for } p=q+2,-p \equiv 2,3 \bmod 4, \\ \bar{H}^{2 a-1}(F ; \mathbb{Z} / 2) & \text { for }(p, q) \text { distinguished, } \\ 0 & \text { otherwise, }\end{cases}
$$

for $p, q \leq 0$. Here $a \geq 2$ so $\bar{H}^{2 a-1}(F ; \mathbb{Z} / 2) \cong(\mathbb{Z} / 2)^{r_{1}-1}$. The given formulas for $K_{*}(F ; \mathbb{Z} / 2)$ follow.

In order to pass to the mod $2 K$-theory of $R$ we need two lemmas.

Lemma 7.6. (a) Restriction of the connecting map $\partial_{1}^{e}$ of the mod 2 étale cohomology localization sequence for $R$ to the kernel of the sign map $\sigma / 2$ determines the following commutative diagram with exact rows:



The vertical maps are injective, and the kernel-cokernel sequence of the maps in the right square can be identified with the following exact sequence:

$$
0 \rightarrow \tilde{H}^{1}(R ; \mathbb{Z} / 2) \rightarrow H^{1}(R ; \mathbb{Z} / 2) \stackrel{\sigma / 2}{\longrightarrow}(\mathbb{Z} / 2)^{r_{1}} \rightarrow \operatorname{Pic}_{+}(R) / 2 \stackrel{\rho / 2}{\longrightarrow} \operatorname{Pic}(R) / 2 \rightarrow 0 .
$$


(b) Restriction of the connecting map $\partial_{2}^{e}$ to the kernel of ${ }_{2} \tau:{ }_{2} \operatorname{Br}(F) \rightarrow(\mathbb{Z} / 2)^{r_{1}}$ determines the following commutative diagram with exact rows:

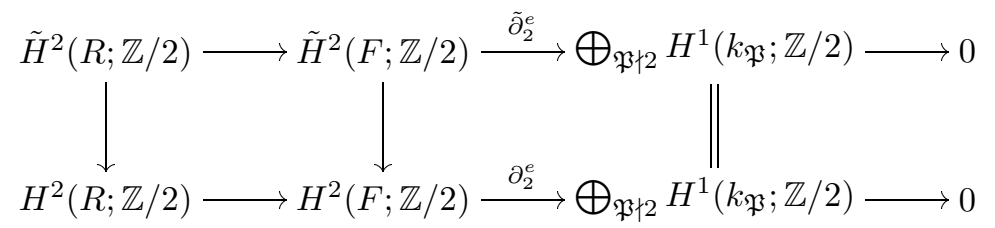

Proof. The localization sequence on the bottom row of the diagram in (a) continues with the bottom row of the diagram in (b). A diagram chase shows that the top rows are also exact. Identifying $H^{1}(R ; \mathbb{Z} / 2)$ with $R^{\times} / 2 \oplus_{2} \operatorname{Pic}(R)$, it follows from the diagram in Definition 7.3 that the horizontal cokernels in diagram (a) are $\mathrm{Pic}_{+}(R) / 2$ and $\operatorname{Pic}(R) / 2$, respectively.

Lemma 7.7. Let $E$ be a p-local field of characteristic zero with valuation ring $\mathcal{O}_{E}$ and separable closure $E^{s}$. Suppose $p \neq 2$. For each $i \geq 0$ the composite

$$
K_{2 i}\left(\mathcal{O}_{E} ; \mathbb{Z} / 2\right) \rightarrow K_{2 i}(E ; \mathbb{Z} / 2) \rightarrow K_{2 i}\left(E^{s} ; \mathbb{Z} / 2\right) \cong \mathbb{Z} / 2
$$

is an isomorphism.

Proof. Let $\mathcal{O}^{h}$ denote the integral closure of $\mathcal{O}_{E}$ in $E^{s}$; it is a complete local ring with separably closed residue field $k^{s}$. Let $k$ be the residue field of $\mathcal{O}_{E}$. By [W1, 4.1(c)] there is a short exact sequence

$$
0 \rightarrow K_{2 i}\left(\mathcal{O}_{E} ; \mathbb{Z} / 2\right) \rightarrow K_{2 i}(E ; \mathbb{Z} / 2) \rightarrow K_{2 i-1}(k ; \mathbb{Z} / 2) \rightarrow 0
$$

and a similar exact sequence for $\mathcal{O}^{h}$, which degenerates by Proposition 1.10 to yield

$$
K_{2 i}\left(\mathcal{O}^{h} ; \mathbb{Z} / 2\right) \cong K_{2 i}\left(E^{s} ; \mathbb{Z} / 2\right) \cong \mathbb{Z} / 2 \text {. }
$$

Therefore it suffices to show that $K_{2 i}\left(\mathcal{O}_{E} ; \mathbb{Z} / 2\right) \rightarrow K_{2 i}\left(\mathcal{O}^{h} ; \mathbb{Z} / 2\right)$ is an isomorphism. But by Proposition 1.10 and Gabber's Rigidity Theorem 3.2 this is just the isomorphism $K_{2 i}(k ; \mathbb{Z} / 2) \cong K_{2 i}\left(k^{s} ; \mathbb{Z} / 2\right)$.

Theorem 7.8. Let $F$ be a real number field, with $R$ its ring of 2-integers. The mod 2 algebraic $K$-groups of $R$ are given (up to extensions) for $n>0$ as follows:

$$
K_{n}(R ; \mathbb{Z} / 2) \cong \begin{cases}\tilde{H}^{2}(R ; \mathbb{Z} / 2) \oplus H^{0}(R ; \mathbb{Z} / 2) & \text { for } n=8 k, \\ H^{1}(R ; \mathbb{Z} / 2) & \text { for } n=8 k+1, \\ H^{2}(R ; \mathbb{Z} / 2) \rtimes H^{0}(R ; \mathbb{Z} / 2) & \text { for } n=8 k+2, \\ (\mathbb{Z} / 2)^{r_{1}-1} \rtimes H^{1}(R ; \mathbb{Z} / 2) & \text { for } n=8 k+3, \\ \operatorname{Pic}_{+}(R) / 2 \rtimes{ }_{2} \operatorname{Br}(R) & \text { for } n=8 k+4, \\ (\mathbb{Z} / 2)^{r_{1}-1} \rtimes \tilde{H}^{1}(R ; \mathbb{Z} / 2) & \text { for } n=8 k+5, \\ \operatorname{Pic}_{+}(R) / 2 \oplus{ }_{2} \mathrm{Br}_{+}(R) & \text { for } n=8 k+6, \\ \tilde{H}^{1}(R ; \mathbb{Z} / 2) & \text { for } n=8 k+7 .\end{cases}
$$

Here $H^{0}(R ; \mathbb{Z} / 2)=\mathbb{Z} / 2, H^{1}(R ; \mathbb{Z} / 2) \cong R^{\times} / 2 \oplus_{2} \operatorname{Pic}(R), H^{2}(R ; \mathbb{Z} / 2) \cong \operatorname{Pic}(R) / 2 \oplus$ ${ }_{2} \operatorname{Br}(R)$ and $\tilde{H}^{2}(R ; \mathbb{Z} / 2) \cong \operatorname{Pic}(R) / 2 \oplus_{2} \mathrm{Br}_{+}(R)$.

Proof. We first consider odd $n=2 i-1$, using Proposition 7.1. When $n=8 k+1$ (so $i \equiv 1 \bmod 4$ ) the edge map for $F$ is an isomorphism, hence so is the induced $\operatorname{map} K_{n}(R ; \mathbb{Z} / 2) \rightarrow H^{1}(R ; \mathbb{Z} / 2)$. 
When $i \equiv 0 \bmod 4$, the edge map for $F$ is injective, hence so is the induced map for $R$. When $i \equiv 2 \bmod 4$, the edge map for $F$ is surjective, hence so is the induced map for $R$.

When $i \equiv 2,3 \bmod 4$ the kernel of the edge map for $F$ is $\bar{H}^{2 a-1}(F ; \mathbb{Z} / 2)$, which maps to zero under $\partial_{1}$ because the composite map factors through $K_{2 i-1}\left(F_{\mathfrak{P}} ; \mathbb{Z} / 2\right)$ and thus through $\bar{H}^{2 a-1}\left(F_{\mathfrak{P}} ; \mathbb{Z} / 2\right)=0$ by naturality. (Recall that $a \geq 2$.) So in these cases the induced map on vertical kernels in (7.1) can be identified with the natural isomorphism $\bar{H}^{2 a-1}(R ; \mathbb{Z} / 2) \cong \bar{H}^{2 a-1}(F ; \mathbb{Z} / 2) \cong(\mathbb{Z} / 2)^{r_{1}-1}$. This establishes the result for $i \equiv 2 \bmod 4$.

When $i \equiv 0,3 \bmod 4$ there is a commutative diagram similar to (7.1) obtained by replacing $H^{1}(R ; \mathbb{Z} / 2)$ with $\tilde{H}^{1}(R ; \mathbb{Z} / 2)$, and $H^{1}(F ; \mathbb{Z} / 2)$ with $\tilde{H}^{1}(F ; \mathbb{Z} / 2)$. The new lower row is an exact sequence by Lemma 7.6(a), the new middle vertical edge map is a surjection, and the new left vertical map is again a surjection by the 5 -lemma. This establishes the result for $i \equiv 0,3 \bmod 4$.

The right vertical isomorphism $\phi$ in the diagram of (7.1) identifies the image of $\partial_{1}$ with the image of $\partial_{1}^{e}$ precomposed with the edge map for $F$. The latter is the image of $\partial_{1}^{e}$ on $H^{1}(F ; \mathbb{Z} / 2)$ when $i \equiv 1,2 \bmod 4$, and the image of $\partial_{1}^{e}$ restricted to $\tilde{H}^{1}(F ; \mathbb{Z} / 2)$ when $i \equiv 0,3 \bmod 4$. Hence we can use the diagram in Definition 7.3 to identify $\operatorname{cok} \partial_{1}$ with $\operatorname{cok} e / 2=\operatorname{Pic}(R) / 2$ when $i \equiv 1,2 \bmod 4$, and with cok $e_{+} / 2=$ $\mathrm{Pic}_{+}(R) / 2$ when $i \equiv 0,3 \bmod 4$.

We now consider even $n$. By Theorem 7.4 the mod 2 Bloch-Lichtenbaum spectral sequence determines injective maps $H^{2}(F ; \mathbb{Z} / 2) \rightarrow K_{2 i-2}(F ; \mathbb{Z} / 2)$ for $i \geq 2$ with $i \equiv 2,3 \bmod 4$, and injective maps $\tilde{H}^{2}(F ; \mathbb{Z} / 2) \rightarrow K_{2 i-2}(F ; \mathbb{Z} / 2)$ for $i \geq 2$ with $i \equiv$ $0,1 \bmod 4$. Likewise, by Theorem 1.15(a) the mod 2 Bloch-Lichtenbaum spectral sequence determines injective maps $H^{2}\left(F_{\mathfrak{P}} ; \mathbb{Z} / 2\right) \rightarrow K_{2 i-2}\left(F_{\mathfrak{P}} ; \mathbb{Z} / 2\right)$. These are compatible with respect to the natural maps induced by the embeddings $F \rightarrow F_{\mathfrak{P}}$.

Hence when $i \equiv 2,3 \bmod 4$ the left square in the following diagram commutes:

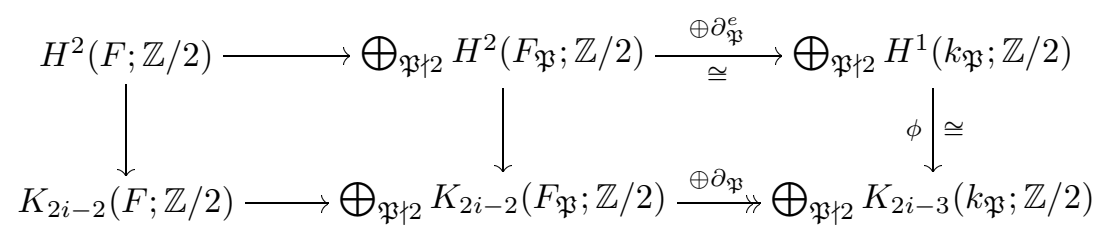

As before the horizontal composites in this diagram are the connecting maps $\partial=\partial_{2}$ and $\partial^{e}=\partial_{2}^{e}$. There is a similar diagram when $i \equiv 0,1 \bmod 4$, replacing $H^{2}(F ; \mathbb{Z} / 2)$ by $\tilde{H}^{2}(F ; \mathbb{Z} / 2)$, and in this case the upper composite is $\tilde{\partial}_{2}^{e}$.

The upper right maps $\partial_{\mathfrak{P}}^{e}$ are isomorphisms by exactness of the mod 2 étale cohomology localization sequence, since $H^{n}\left(\mathcal{O}_{F_{\mathfrak{P}}} ; \mathbb{Z} / 2\right) \cong H^{n}\left(k_{\mathfrak{P}} ; \mathbb{Z} / 2\right)=0$ for $n=2,3$ by Proposition 1.11. Hence there is a unique map $\phi_{\mathfrak{P}}: H^{1}\left(k_{\mathfrak{P}} ; \mathbb{Z} / 2\right) \rightarrow$ $K_{2 i-1}\left(k_{\mathfrak{P}} ; \mathbb{Z} / 2\right)$ for each $\mathfrak{P} \nmid 2$, such that their direct sum $\phi=\bigoplus_{\mathfrak{P}} \phi_{\mathfrak{P}}$ makes the right square in (7.9) commute.

We claim that the maps $\phi_{\mathfrak{P}}$ are isomorphisms. The source and target groups are both isomorphic to $\mathbb{Z} / 2$, so it suffices to show that each map $\phi_{\mathfrak{P}}$ is nonzero, or equivalently that the composite

$$
H^{2}\left(F_{\mathfrak{P}} ; \mathbb{Z} / 2\right) \rightarrow K_{2 i-2}\left(F_{\mathfrak{P}} ; \mathbb{Z} / 2\right) \stackrel{\partial_{\mathfrak{P}}}{\longrightarrow} K_{2 i-3}\left(k_{\mathfrak{P}} ; \mathbb{Z} / 2\right)
$$

is nonzero. By exactness of the mod $2 K$-theory localization sequence we must prove that the (nonzero) left injection here does not factor through $K_{2 i-2}\left(\mathcal{O}_{F_{\mathfrak{P}}} ; \mathbb{Z} / 2\right)$. But 
consider the composites

$$
\begin{aligned}
K_{2 i-2}\left(\mathcal{O}_{F_{\mathfrak{P}}} ; \mathbb{Z} / 2\right) & \rightarrow K_{2 i-2}\left(F_{\mathfrak{P}} ; \mathbb{Z} / 2\right) \rightarrow K_{2 i-2}\left(F_{\mathfrak{P}}^{s} ; \mathbb{Z} / 2\right), \\
H^{2}\left(F_{\mathfrak{P}} ; \mathbb{Z} / 2\right) & \rightarrow K_{2 i-2}\left(F_{\mathfrak{P}} ; \mathbb{Z} / 2\right) \rightarrow K_{2 i-2}\left(F_{\mathfrak{P}}^{s} ; \mathbb{Z} / 2\right)
\end{aligned}
$$

where $F_{\mathfrak{P}}^{s}$ is a separable closure of $F_{\mathfrak{P}}$. By Lemma 7.7 the first composite is an isomorphism of nontrivial groups, while the second composite factors through $H^{2}\left(F_{\mathfrak{P}}^{s} ; \mathbb{Z} / 2\right)=0$ by naturality of the Bloch-Lichtenbaum spectral sequence, hence is zero. This contradicts a factorization of the map from $H^{2}\left(F_{\mathfrak{P}} ; \mathbb{Z} / 2\right)$ through $K_{2 i-2}\left(\mathcal{O}_{F_{\mathfrak{P}}} ; \mathbb{Z} / 2\right)$. Hence each map $\phi_{\mathfrak{P}}$ is nonzero, and an isomorphism, as claimed.

We have thus established the commutative diagram

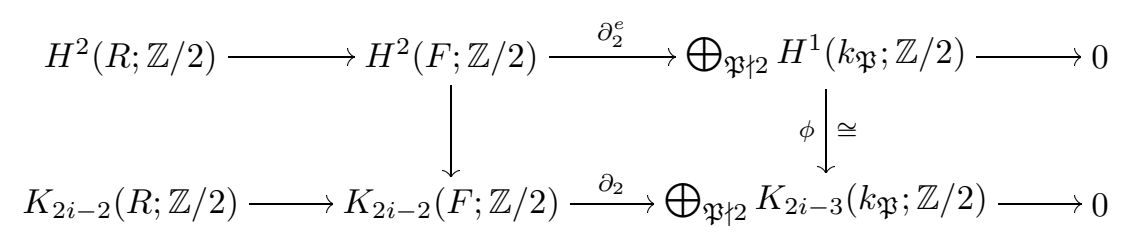

when $i \equiv 2,3 \bmod 4$, and the commutative diagram

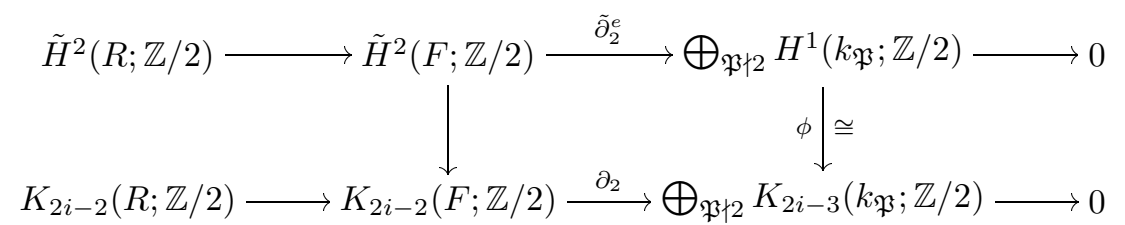

when $i \equiv 0,1 \bmod 4$. In both cases the middle vertical map is the injection determined by the Bloch-Lichtenbaum spectral sequence, the upper row is an exact sequence by Lemma 7.6(b), and the lower row is part of the $K$-theory localization sequence.

Hence we have an injection $\operatorname{ker} \partial_{2}^{e} \rightarrow \operatorname{ker} \partial_{2}$ for $i \equiv 2,3 \bmod 4$, and an injection $\operatorname{ker} \tilde{\partial}_{2}^{e} \rightarrow \operatorname{ker} \partial_{2}$ for $i \equiv 0,1 \bmod 4$. These maps are isomorphisms for $i \equiv 0,3 \bmod 4$, and we can identify their cokernels with $H^{0}(R ; \mathbb{Z} / 2) \cong H^{0}(F ; \mathbb{Z} / 2)$ for $i \equiv 1,2 \mathrm{mod}$ 4. Finally ker $\partial_{2}^{e} \cong{ }_{2} \operatorname{Br}(R)$ and ker $\tilde{\partial}_{2}^{e} \cong{ }_{2} \operatorname{Br}_{+}(R)$.

The mod 2 algebraic $K$-theory localization sequence determines a short exact sequence

$$
0 \rightarrow \operatorname{cok} \partial_{1} \rightarrow K_{2 i-2}(R ; \mathbb{Z} / 2) \rightarrow \operatorname{ker} \partial_{2} \rightarrow 0 .
$$

Combining these results we obtain isomorphisms

$$
\begin{aligned}
& K_{2 i-2}(R ; \mathbb{Z} / 2) \cong \mathrm{Pic}_{+}(R) / 2 \rtimes_{2} \mathrm{Br}_{+}(R), \text { resp. } \\
& K_{2 i-2}(R ; \mathbb{Z} / 2) \cong \mathrm{Pic}_{+}(R) / 2 \rtimes_{2} \operatorname{Br}(R)
\end{aligned}
$$

when $i \equiv 0 \bmod 4$, resp. $i \equiv 3 \bmod 4$, and short exact sequences

$$
\begin{aligned}
& 0 \rightarrow \operatorname{Pic}(R) / 2 \rightarrow K_{2 i-2}(R ; \mathbb{Z} / 2) \rightarrow{ }_{2} \mathrm{Br}_{+}(R) \rtimes H^{0}(R ; \mathbb{Z} / 2) \rightarrow 0, \text { resp. } \\
& 0 \rightarrow \operatorname{Pic}(R) / 2 \rightarrow K_{2 i-2}(R ; \mathbb{Z} / 2) \rightarrow{ }_{2} \operatorname{Br}(R) \rtimes H^{0}(R ; \mathbb{Z} / 2) \rightarrow 0
\end{aligned}
$$

when $i \equiv 1 \bmod 4$, resp. $i \equiv 2 \bmod 4$. This determines the groups $K_{n}(R ; \mathbb{Z} / 2)$ up to extensions.

We can say a little more about the extensions, by the following arguments.

When $n=8 k+2$ the exact sequence

$$
0 \rightarrow K_{n+1}\left(R ; \mathbb{Z} / 2^{\infty}\right) / 2 \rightarrow K_{n}(R ; \mathbb{Z} / 2) \rightarrow{ }_{2} K_{n}\left(R ; \mathbb{Z} / 2^{\infty}\right) \rightarrow 0
$$


and the isomorphisms $H^{2}(R ; \mathbb{Z} / 2) \cong H^{1}(R ; W(4 k+2)) / 2 \cong K_{8 k+3}\left(R ; \mathbb{Z} / 2^{\infty}\right) / 2$ and $H^{0}(R ; \mathbb{Z} / 2) \cong{ }_{2} H^{0}(R ; W(4 k+1)) \cong{ }_{2} K_{8 k+2}\left(R ; \mathbb{Z} / 2^{\infty}\right)$ (from Proposition 4.6 and Theorem 6.9) determine the extension a little more precisely, as proclaimed.

When $n=8 k$, the same exact sequence and corresponding isomorphisms produce a surjection from $K_{8 k}(R ; \mathbb{Z} / 2)$ onto $H^{0}(R ; \mathbb{Z} / 2)$, with kernel

$$
H^{1}(R ; W(4 k+1)) / 2
$$

This group is identified with the kernel of the natural map

$$
H^{2}(R ; \mathbb{Z} / 2) \rightarrow{ }_{2} H^{2}(R ; W(4 k+1)) \cong(\mathbb{Z} / 2)^{r_{1}},
$$

which by naturality equals the surjection $\alpha^{2}$ with kernel

$$
\tilde{H}^{2}(R ; \mathbb{Z} / 2) \cong \operatorname{Pic}(R) / 2 \oplus_{2} \mathrm{Br}_{+}(R)
$$

In fact, $H^{0}(R ; \mathbb{Z} / 2) \cong \mathbb{Z} / 2$ is a summand of $K_{8 k}(R ; \mathbb{Z} / 2)$. To see this, recall from section 5 that the map $\pi_{8 k}\left(Q S^{0} ; \mathbb{Z} / 2\right) \rightarrow K_{8 k}(\mathbb{R} ; \mathbb{Z} / 2) \cong H^{0}(\mathbb{R} ; \mathbb{Z} / 2)$ is nontrivial on the element $\beta_{8 k}$. Since this map factors through the surjection $K_{8 k}(R ; \mathbb{Z} / 2) \rightarrow$ $H^{0}(R ; \mathbb{Z} / 2) \cong \mathbb{Z} / 2$, it splits it.

We saw in Theorem 6.14(b) that $K_{8 k+5}(R)\{2\}=0$ in the real case. Hence $K_{8 k+6}(R ; \mathbb{Z} / 2)$ has exponent 2 , so the extension in this degree is trivial. This completes the proof of Theorem 7.8.

Corollary 7.10. Let $S K_{n}(R ; \mathbb{Z} / 2)$ be the kernel of the natural map $K_{n}(R ; \mathbb{Z} / 2) \rightarrow$ $K_{n}(F ; \mathbb{Z} / 2)$. Then

$$
S K_{n}(R ; \mathbb{Z} / 2) \cong \begin{cases}0 & \text { for } n \text { odd }, \\ \operatorname{Pic}(R) / 2 & \text { for } n \equiv 0,2 \bmod 8 \\ \operatorname{Pic}_{+}(R) / 2 & \text { for } n \equiv 4,6 \bmod 8\end{cases}
$$

Proof. Compare Theorems 7.4 and 7.8.

Theorem 7.11. Let $F$ be a real number field with $r_{1}>0$ real embeddings and $r_{2}$ pairs of complex embeddings. Let $R$ be its ring of 2-integers, let $s=\# S$ be the number of primes of $F$ dividing 2, let $t$ be the 2-rank of $\operatorname{Pic}(R)$ and let $u$ be the 2 -rank of $\mathrm{Pic}_{+}(R)$. Let $n \geq 1$, and let $\log _{2}(i)$ denote the 2 -adic valuation of $i$. Then

$$
\log _{2}\left(\# K_{n}(R ; \mathbb{Z} / 2)\right)= \begin{cases}s+t & \text { for } n=8 k, \\ r_{1}+r_{2}+s+t & \text { for } n=8 k+1, \\ r_{1}+s+t & \text { for } n=8 k+2, \\ 2 r_{1}+r_{2}+s+t-1 & \text { for } n=8 k+3, \\ r_{1}+s+u-1 & \text { for } n=8 k+4, \\ r_{1}+r_{2}+s+u-1 & \text { for } n=8 k+5, \\ s+u-1 & \text { for } n=8 k+6, \\ r_{2}+s+u & \text { for } n=8 k+7 .\end{cases}
$$


Hence the number of cyclic summands in $K_{n}(R)\{2\}$ equals

$$
\begin{cases}s+t-1 & \text { for } n=8 k, \\ 1 & \text { for } n=8 k+1 \\ r_{1}+s+t-1 & \text { for } n=8 k+2 \\ r_{1} & \text { for } n=8 k+3 \\ s+u-1 & \text { for } n=8 k+4 \\ 0 & \text { for } n=8 k+5 \\ s+u-1 & \text { for } n=8 k+6 \\ 1 & \text { for } n=8 k+7\end{cases}
$$

Proof. Clearly, $\log _{2}\left(\# H^{0}(R ; \mathbb{Z} / 2)\right)=1$ and $\log _{2}\left(\# \bar{H}^{2 a-1}(R ; \mathbb{Z} / 2)\right)=r_{1}-1$. Also $R^{\times} / 2 \cong(\mathbb{Z} / 2)^{r_{1}+r_{2}+s}$ by Dirichlet's unit theorem, $\operatorname{Pic}(R) / 2 \cong{ }_{2} \operatorname{Pic}(R) \cong(\mathbb{Z} / 2)^{t}$ and $\operatorname{Pic}_{+}(R) / 2 \cong{ }_{2} \operatorname{Pic}_{+}(R) \cong(\mathbb{Z} / 2)^{u}$, so $\log _{2}\left(\# H^{1}(R ; \mathbb{Z} / 2)\right)=r_{1}+r_{2}+s+t$. Also, by Lemma 7.6 (a) we have $\log _{2}\left(\# \tilde{H}^{1}(R ; \mathbb{Z} / 2)\right)=r_{2}+s+u$. Finally ${ }_{2} \operatorname{Br}(R) \cong$ $(\mathbb{Z} / 2)^{r_{1}+s-1}$, so $\log _{2}\left(\# H^{2}(R ; \mathbb{Z} / 2)\right)=r_{1}+s+t-1$, and from Lemma 7.6(b) we deduce $\log _{2}\left(\# \tilde{H}^{2}(R ; \mathbb{Z} / 2)\right)=s+t-1$.

Combined with Theorem 7.8 this gives us the orders of $K_{n}(R ; \mathbb{Z} / 2)$ for all $n \geq 1$, and the listed 2-valuations. Using the known rational ranks from 6.2 , we can now inductively determine the orders of (tors $\left.K_{n}(R)\right) / 2$ by starting with the order of ${ }_{2} K_{0}(R)={ }_{2} \operatorname{Pic}(R)$.

Corollary 7.12. Let $F$ be a real number field. Let $t$ and $u$ be the 2 -rank of $\operatorname{Pic}(R)$ and $\mathrm{Pic}_{+}(R)$, respectively, and let $j=u-t$ be the signature defect of $R$. Then for $k \geq 0$ :

(a) $K_{8 k+3}(R)\{2\} \cong(\mathbb{Z} / 2)^{r_{1}} \rtimes \mathbb{Z} / w_{4 k+2}(F)$ has $r_{1}$ cyclic summands. The extension is nontrivial, so

$$
K_{8 k+3}(R)\{2\} \cong(\mathbb{Z} / 2)^{r_{1}-1} \oplus \mathbb{Z} /\left(2 w_{4 k+2}(F)\right) .
$$

(b) There are $r_{1}-j-1$ cyclic factors with nontrivial extensions in the exact sequence

$$
0 \rightarrow(\mathbb{Z} / 2)^{r_{1}-1} \rightarrow K_{8 k+5}\left(R ; \mathbb{Z} / 2^{\infty}\right) \rightarrow\left(\mathbb{Z} / 2^{\infty}\right)^{r_{1}+r_{2}} \oplus H^{2}\left(R ; \mathbb{Z}_{2}(4 k+3)\right) \rightarrow 0 .
$$

Hence there is an exact sequence

$$
0 \rightarrow(\mathbb{Z} / 2)^{\rho} \rightarrow K_{8 k+4}(R)\{2\} \rightarrow H^{2}\left(R ; \mathbb{Z}_{2}(4 k+3)\right) \rightarrow 0
$$

with $\rho-j$ cyclic factors with nontrivial extensions. Here $j \leq \rho<r_{1}$.

(c) There are $j$ cyclic factors with nontrivial extensions in the short exact sequence

$$
0 \rightarrow K_{8 k+6}(R)\{2\} \rightarrow H^{2}\left(R ; \mathbb{Z}_{2}(4 k+4)\right) \rightarrow(\mathbb{Z} / 2)^{r_{1}} \rightarrow 0 .
$$

Proof. We combine Theorem 6.14 with the numbers from Proposition 6.13 and Theorem 7.11. In each case the discrepancy between the 2-rank of an extension $A \rtimes B$ and the sum of the 2-rank of $A$ and the 2-rank of $B$ equals the number of cyclic summands of $A \rtimes B$ that extend nontrivially from $B$ to $A$. This proves (a) and (c).

Case (b) merits further discussion. The group $K_{8 k+5}\left(R ; \mathbb{Z} / 2^{\infty}\right)$ is an extension of $\left(\mathbb{Z} / 2^{\infty}\right)^{r_{1}+r_{2}} \oplus H^{2}\left(R ; \mathbb{Z}_{2}(4 k+3)\right)$ by $(\mathbb{Z} / 2)^{r_{1}-1}$, and has $K_{8 k+4}(R)\{2\}$ as its maximal finite quotient. Here the finite group $H^{2}\left(R ; \mathbb{Z}_{2}(4 k+3)\right)$ has 2-rank $s+t-1$ and $K_{8 k+4}(R)\{2\}$ is finite with 2-rank $s+u-1$, so there are precisely $r_{1}-j-1$ 
cyclic factors with nontrivial extensions in $K_{8 k+5}\left(R ; \mathbb{Z} / 2^{\infty}\right)$. Suppose $x$ of these are extensions from the divisible summands $\left(\mathbb{Z} / 2^{\infty}\right)^{r_{1}+r_{2}}$. Then $\rho=r_{1}-x-1$, and the remaining $r_{1}-j-x-1=\rho-j$ extensions come from the finite summand $H^{2}\left(R ; \mathbb{Z}_{2}(4 k+3)\right)$. In particular $j \leq \rho<r_{1}$.

\section{Appendix A: COHOMOLOGicAl Version OF THE LICHTENBAUM CONJECTURE AT THE PRIME 2 By Manfred Kolster}

The purpose of this appendix is to prove the following result, which was used in the main part of the paper by Rognes and Weibel.

Theorem A.1. Let $F$ be a totally real number field. If the 2-adic Main Conjecture holds for the field $F$ and the trivial character, then for any even integer $n \geq 2$ :

$$
\zeta_{F}(1-n) \sim_{2} \frac{\left|H_{\text {ét }}^{2}\left(\mathcal{O}_{F}\left[\frac{1}{2}\right] ; \mathbb{Z}_{2}(n)\right)\right|}{\left|H_{\text {ét }}^{1}\left(\mathcal{O}_{F}\left[\frac{1}{2}\right] ; \mathbb{Z}_{2}(n)\right)\right|} .
$$

Here we use the notation $a \sim_{2} b$ to indicate that both sides have the same 2-adic valuation. In the special case $n=2$ the theorem was proved in $[\mathrm{Ko}, \mathrm{Ngu}]$, and in particular the proof given in [Ngu] can be easily extended to the more general situation considered above. We present a slight simplification of the method used in $[\mathrm{Ngu}]$, similar to the corresponding proof for odd $p$ given in [KNF].

The 2-adic Main Conjecture was proved by Wiles ( $c f$. [Wi]) for all real onedimensional 2-adic Artin characters of type $S$ over the field $\mathbb{Q}$ of rational numbers. To extend the relation in Theorem A.1 to all Abelian number fields we prove a kind of "Going-Up" result for the Main Conjecture, which seems to be part of the general folklore in Iwasawa-theory. I am very grateful to R. Greenberg for some stimulating discussions on this subject, which led to a proof of the following:

Theorem A.2. Assume that the 2-adic Main Conjecture holds for all real 1-dimensional 2-adic Artin characters of type $S$ over a totally real number field $K$. Let $F$ be a finite totally real extension of $K$. Then the 2-adic Main Conjecture holds for all those real 1-dimensional 2-adic Artin characters $\psi$ of type $S$ over $F$, for which the field $F_{\psi}$ is Abelian over $K$.

As an immediate consequence we obtain:

Corollary A.3. The formula in Theorem A.1 holds for all totally real Abelian number fields.

We first recall one of the formulations of the Main Conjecture in Iwasawa theory due to Greenberg ( $c f$. [Gr2, Fed, Gri, Wi]). Throughout $F$ denotes a totally real number field with ring of integers $\mathcal{O}_{F}$. Let $p$ be a prime number and let $\psi$ denote a real one-dimensional $p$-adic Artin character for $F$, i.e., the field extension $F_{\psi}$ of $F$ attached to $\psi$ is real and Abelian over $F$. Deligne and Ribet ( $c f$. [DR]) proved the existence of a $p$-adic $L$-function $L_{p}(s, \psi)$, continuous for $s \in \mathbb{Z}_{p} \backslash\{1\}$ and also at $s=1$ if $\psi$ is nontrivial, which interpolates the values of $L$-functions via

$$
L_{p}(1-n, \psi)=L\left(1-n, \psi \omega^{-n}\right) \prod_{\mathfrak{P} \mid p}\left(1-\psi \omega^{-n}(\mathfrak{P}) N \mathfrak{P}^{n-1}\right)
$$

for $n \geq 1$. Here $\omega$ denotes the Teichmüller character

$$
\omega: \operatorname{Gal}\left(F\left(\mu_{2 p}\right) / F\right) \rightarrow \mathbb{Z}_{p}^{\times} .
$$


In particular, for $n \geq 2$ we always have

$$
L_{p}(1-n, \psi) \quad \sim_{p} \quad L\left(1-n, \psi \omega^{-n}\right) .
$$

The character $\psi$ is called of type $S$ if $F_{\psi} \cap F_{\infty}=F$ and of type $W$ if $F_{\psi} \subset F_{\infty}$. Here $F_{\infty}$ denotes the cyclotomic $\mathbb{Z}_{p}$-extension of $F$. Let $\Gamma=\operatorname{Gal}\left(F_{\infty} / F\right)$ and let $\gamma$ denote a topological generator of $\Gamma$. Let $\mathcal{O}_{\psi}=\mathbb{Z}_{p}[\psi]$. There exists a unique power series $(c f .[\mathrm{DR}]) G_{\psi}(T) \in \mathcal{O}_{\psi}[[T]]$, such that

$$
L_{p}(1-s, \psi)=G_{\psi}\left(\kappa(\gamma)^{s}-1\right) / H_{\psi}\left(\kappa(\gamma)^{s}-1\right),
$$

where

$$
H_{\psi}(T)= \begin{cases}\psi(\gamma)(1+T)-1 & \text { if } \psi \text { is of type } W \\ 1 & \text { otherwise }\end{cases}
$$

and $\kappa$ denotes the cyclotomic character. By the Weierstrass preparation theorem the power series $G_{\psi}(T)$ can be written uniquely as

$$
G_{\psi}(T)=\pi^{\mu\left(G_{\psi}\right)} G_{\psi}^{*}(T) u(T),
$$

where $\pi$ denotes a uniformizing parameter in $\mathcal{O}_{\psi}, G_{\psi}^{*}(T)$ is a distinguished polynomial and $u(T)$ is a unit power series.

In order to formulate the Main Conjecture we have to introduce a few more notations. Let $N$ be an arbitrary number field and let $S$ consist of the infinite primes of $N$ and the primes above $p$. Let $\Omega_{S}(N)$ denote the maximal algebraic extension of $N$ that is unramified outside of primes in $S$, and let $G_{S}(N)=\operatorname{Gal}\left(\Omega_{S}(N) / N\right)$. Let $M_{\infty}(N)$ denote the maximal Abelian $p$-extension of $N_{\infty}$ contained in $\Omega_{S}(N)$. We note that it is important to allow ramification at infinite primes, which of course only intervenes if $p=2$. The central Iwasawa module is now $\mathcal{X}(N)=\operatorname{Gal}\left(M_{\infty}(N) / N_{\infty}\right)$. If $N$ is totally real, then $\mathcal{X}(N)$ is a Noetherian torsion $\Lambda$-module, $\Lambda=\mathbb{Z}_{p}[[T]]$, without nontrivial finite submodules ( $c f$. [Iwa2]). Let us consider the case that $N=F_{\psi}$, and assume that $\psi$ is of type $S$. The $\operatorname{Galois} \operatorname{group} \operatorname{Gal}\left(N_{\infty} / F\right)$ is then isomorphic to $\Gamma \times \Delta$, where $\Delta=\operatorname{Gal}(N / F)$, and acts on $\mathcal{X}(N)$ via conjugation. Let

$$
\mathcal{X}(N)^{\psi}=\left\{x \in \mathcal{X}(N) \otimes \mathcal{O}_{\psi} \mid \sigma(x)=\psi(\sigma) x \quad \text { for all } \sigma \in \Delta\right\}
$$

and let $g_{\psi}(T) \in \mathcal{O}_{\psi}[[T]]$ denote the characteristic polynomial of $\mathcal{X}(N)^{\psi}$, which we can write as

$$
g_{\psi}(T)=\pi^{\mu\left(g_{\psi}\right)} g_{\psi}^{*}(T)
$$

with $g_{\psi}^{*}(T)$ distinguished. One way of formulating the Main Conjecture in Iwasawa Theory is the following ( $c f$. [Gr2]):

Main Conjecture. Let $F$ be a totally real number field, $p$ a prime and $\psi$ a real one-dimensional p-adic Artin character for $F$ of type S. Then

$$
G_{\psi}^{*}(T)=g_{\psi}^{*}(T) .
$$

The Main Conjecture was proved for odd primes $p$ and all totally real number fields and for the prime 2 and the field of rational numbers by Wiles in [Wi]. A different proof for the prime 2 and the field of rational numbers is due to Greither $([\mathrm{Gri}])$. 
Remark A.4. (a) Let $W(N)=\mathcal{X}(N) \otimes \overline{\mathbb{Q}}_{p}$. Then $W(N)$ is a finite-dimensional vector space and $g_{\psi}^{*}(T)$ is the characteristic polynomial of $\gamma-1$ acting on the $\psi$-eigenspace $W(N)^{\psi}$, since tensoring $\mathcal{X}(N)^{\psi}$ by $\overline{\mathbb{Q}}_{p}$ removes $\Lambda$-torsion. The formulation in [Gr2] is given in terms of $W(N)^{\psi}$.

(b) A stronger version of the Main Conjecture (First Form in [Ws]) would include a precise relation between the $\mu$-invariants of $G_{\psi}(T)$ and $g_{\psi}(T)$ as suggested by Greenberg in [Gr2]. For odd primes $p$ and characters of order prime to $p$ Wiles has shown that both $\mu$-invariants are equal. Of course they are conjectured to be trivial for odd $p$, this being true for Abelian number fields. For the prime 2 these invariants are nontrivial in general. However, in the special situation we are interested in, namely that $\psi$ is the trivial character, the two $\mu$-invariants are equal as a consequence of the analytic class number formula (see [Fed, Gr2]).

Proof of Theorem A.1. Let $\mathcal{X}=\mathcal{X}(F)$ and let us simply write $G(T)$ and $g(T)$ for $G_{\mathbf{1}}(T)$ and $g_{\mathbf{1}}(T)$, respectively. Since $n$ is even and $\geq 2$ we have

$$
\zeta_{F}(1-n) \sim_{2} \quad L_{2}(1-n, \mathbf{1})=\frac{G\left(\kappa(\gamma)^{n}-1\right)}{\kappa(\gamma)^{n}-1} .
$$

Now clearly $\kappa(\gamma)^{n}-1 \sim_{2} w_{n}(F) \sim_{2}\left|H_{\text {ét }}^{1}\left(\mathcal{O}_{F}\left[\frac{1}{2}\right] ; \mathbb{Z}_{2}(n)\right)\right|$. Remark A.4(b) and the Main Conjecture imply that

$$
G\left(\kappa(\gamma)^{n}-1\right) \quad \sim_{2} \quad g\left(\kappa(\gamma)^{n}-1\right) .
$$

Now $g\left(\kappa\left(\gamma^{n}\right)(1+T)-1\right)$ is the characteristic polynomial of $\mathcal{X}(-n)$. The nonvanishing of the zeta-function of $F$ at $1-n$ now implies that the groups $\mathcal{X}(-n)_{\Gamma}$ and $\mathcal{X}(-n)^{\Gamma}$ are finite (cf. [Coa, Lemma 9, Appendix]). Hence $\mathcal{X}(-n)^{\Gamma}$ is trivial, since $\mathcal{X}$ has no nontrivial finite submodules. Therefore, again by [Coa, Lemma 9 , Appendix], the order of $\mathcal{X}(-n)_{\Gamma}$ has the same 2-adic valuation as $g\left(\kappa(\gamma)^{n}-1\right)$. We are therefore reduced to showing that $\mathcal{X}(-n)_{\Gamma}$ has order $\left|H_{\text {êt }}^{2}\left(\mathcal{O}_{F}\left[\frac{1}{2}\right] ; \mathbb{Z}_{2}(n)\right)\right|$.

Consider the Pontryagin dual:

$$
\left(\mathcal{X}(-n)_{\Gamma}\right)^{\#} \cong \operatorname{Hom}\left(\mathcal{X}, \mathbb{Q}_{2} / \mathbb{Z}_{2}(n)\right)^{\Gamma}
$$

Since $n$ is even, $\mathbb{Q}_{2} / \mathbb{Z}_{2}(n)$ is a trivial module for $G_{S}\left(F_{\infty}\right)$, and hence

$$
\operatorname{Hom}\left(\mathcal{X}, \mathbb{Q}_{2} / \mathbb{Z}_{2}(n)\right)=H^{1}\left(G_{S}\left(F_{\infty}\right) ; \mathbb{Q}_{2} / \mathbb{Z}_{2}(n)\right) .
$$

Since the cohomology groups $H^{1}\left(\Gamma ; \mathbb{Q}_{2} / \mathbb{Z}_{2}(n)\right)$ and $H^{2}\left(\Gamma ; \mathbb{Q}_{2} / \mathbb{Z}_{2}(n)\right)$ vanish, the Hochschild-Serre spectral sequence gives an isomorphism

$$
H^{1}\left(G_{S}(F) ; \mathbb{Q}_{2} / \mathbb{Z}_{2}(n)\right) \cong H^{1}\left(G_{S}\left(F_{\infty}\right) ; \mathbb{Q}_{2} / \mathbb{Z}_{2}(n)\right)^{\Gamma} .
$$

Now the group $H^{1}\left(G_{S}(F) ; \mathbb{Q}_{2} / \mathbb{Z}_{2}(n)\right)$ is isomorphic to the étale cohomology group $H_{\text {ét }}^{1}\left(\mathcal{O}_{F}\left[\frac{1}{2}\right] ; \mathbb{Q}_{2} / \mathbb{Z}_{2}(n)\right)(c f$. [Z, Prop. 3.3.1]), which - being finite - is isomorphic to $H_{\text {ét }}^{2}\left(\mathcal{O}_{F}\left[\frac{1}{2}\right] ; \mathbb{Z}_{2}(n)\right)$. This proves the claim.

We now turn to the proof of Theorem A.2:

Proof of Theorem A.2. Let $N=F_{\psi}$ and let $G=\operatorname{Gal}(N / K), H=\operatorname{Gal}(N / F)$. Since $G$ is Abelian, the induced character $\operatorname{Ind}_{H}^{G}(\psi)$ is of the form $\operatorname{Ind}_{H}^{G}(\psi)=\sum \chi$, where $\chi$ runs through those characters of $G$, which restrict to $\psi$. Let $\gamma$ denote a topological 
generator of $\Gamma=\operatorname{Gal}\left(K_{\infty} / K\right)$. Let $K_{e}=F \cap K_{\infty}$ have degree $2^{e}$ over $K$. Then $\gamma_{e}:=\gamma^{2^{e}}$ is a topological generator of $\operatorname{Gal}\left(F_{\infty} / F\right)$, and we have

$$
L_{p}(1-s, \psi)=\prod_{\chi} L_{p}(1-s, \chi),
$$

hence

$$
\frac{G_{\psi}\left(\kappa\left(\gamma_{e}\right)^{s}-1\right)}{H_{\psi}\left(\kappa\left(\gamma_{e}\right)^{s}-1\right)}=\prod_{\chi} \frac{G_{\chi}\left(\kappa(\gamma)^{s}-1\right)}{H_{\chi}\left(\kappa(\gamma)^{s}-1\right)}
$$

If we let $T=\kappa(\gamma)^{s}-1$, then clearly $T_{e}:=(1+T)^{2^{e}}-1=\kappa\left(\gamma_{e}\right)^{s}-1$. It is easy to verify that

$$
H_{\psi}\left(T_{e}\right)=\prod_{\chi} H_{\chi}(T)
$$

hence we obtain

$$
G_{\psi}\left(T_{e}\right)=\prod_{\chi} G_{\chi}(T)
$$

and therefore also

$$
G_{\psi}^{*}\left(T_{e}\right)=\prod_{\chi} G_{\chi}^{*}(T)
$$

If $e=0$, then the polynomial $g_{\psi}^{*}(T)$ also behaves well under induction ( $c f$. Proposition 1 in [Gr3]), and therefore by inducing to $\operatorname{Gal}\left(N / K_{e}\right)$ we may assume that $\psi$ is a character over $F=K_{e}$. Let $\chi$ be a lift of $\psi$ to $G$, and let $\chi=\lambda \rho$ be a decomposition of $\chi$ into a product of a character $\lambda$ of type $S$ and a character $\rho$ of type $W$. We note that in general $\lambda$ and $\rho$ are not characters of $G$. We have $G_{\chi}^{*}(T) \sim G_{\lambda}^{*}(\rho(\gamma)(1+T)-1)$, where $\sim$ indicates that both sides differ by a root of unity. Since by assumption the Main Conjecture holds for $\lambda$ we have

$$
G_{\lambda}^{*}(\rho(\gamma)(1+T)-1)=g_{\lambda}^{*}(\rho(\gamma)(1+T)-1)
$$

Any other lift $\chi^{\prime}$ of $\psi$ is of the form $\chi^{\prime}=\chi \rho^{\prime}$, where $\rho^{\prime}$ is a character of $\operatorname{Gal}\left(K_{e} / K\right)$. Therefore

$$
G_{\psi}^{*}\left(T_{e}\right) \sim \prod_{\rho^{\prime}} g_{\lambda}^{*}\left(\rho \rho^{\prime}(\gamma)(1+T)-1\right)=\prod_{\zeta} g_{\lambda}^{*}(\zeta \rho(\gamma)(1+T)-1),
$$

where the products run over all characters $\rho^{\prime}$ of $\operatorname{Gal}\left(K_{e} / K\right)$ and all $2^{e}$ th roots of unity $\zeta$, respectively. Let $a_{1}, \ldots, a_{r}$ denote the roots of $g_{\lambda}^{*}(T)$. Then

$$
g_{\lambda}^{*}(\zeta \rho(\gamma)(1+T)-1)=\prod_{i=1}^{r}\left(\zeta \rho(\gamma)(1+T)-\left(1+a_{i}\right)\right) .
$$

Taking the product over all $\zeta$ yields

$$
G_{\psi}^{*}\left(T_{e}\right) \sim \prod_{i=1}^{r}\left(\rho(\gamma)^{2^{e}}(1+T)^{2^{e}}-\left(1+a_{i}\right)^{2^{e}}\right) \sim \prod_{i=1}^{r}\left(T_{e}-\left(\rho(\gamma)^{-2^{e}}\left(1+a_{i}\right)^{2^{e}}-1\right)\right),
$$

hence $\rho(\gamma)^{-2^{e}}\left(1+a_{i}\right)^{2^{e}}-1, i=1, \ldots, r$, are precisely the roots of the polynomial $G_{\psi}^{*}(T)$. Let us now consider the polynomial $g_{\psi}^{*}(T)$ : Let $L=K_{\lambda}$. Clearly $L_{\infty}=N_{\infty}$, and the representation space $W(N)^{\psi}$ equals $W(L)^{\lambda}$. We may identify $\operatorname{Gal}\left(L_{\infty} / K\right)$ with $\Delta \times \Gamma$, where $\Delta=\operatorname{Gal}(L / K)$. Now $\operatorname{Gal}\left(L_{\infty} / N\right)$ is a subgroup of 
$\operatorname{Gal}\left(L_{\infty} / K\right)$ isomorphic to $\operatorname{Gal}\left(K_{\infty} / K_{e}\right)$, hence has a topological generator $\gamma_{N}$ of the form

$$
\gamma_{N}=\delta \gamma_{e}
$$

for some $\delta \in \Delta$. The characteristic polynomial of $\gamma_{N}-1$ acting on $W(L)^{\lambda}$ is $g_{\psi}^{*}(T)$. Since $\delta$ acts on $W(L)^{\lambda}$ via $\lambda(\delta)$ the action of $\gamma_{N}$ is the same as the action of $\lambda(\delta) \gamma_{e}$. Now

$$
1=\chi\left(\gamma_{N}\right)=\chi(\delta) \chi\left(\gamma_{e}\right)=\lambda(\delta) \rho(\gamma)^{2^{e}},
$$

which yields $\lambda(\delta)=\rho(\gamma)^{-2^{e}}$. Now clearly the zeroes of the characteristic polynomial of $\rho(\gamma)^{-2^{e}} \gamma^{2^{e}}-1$ acting on $W(L)^{\lambda}$ are precisely of the form $\rho(\gamma)^{-2^{e}}(1+\alpha)^{2^{e}}-1$ with $\alpha$ a zero of $g_{\lambda}^{*}(T)$, hence $G_{\psi}^{*}(T)$ and $g_{\psi}^{*}(T)$ have the same zeroes, hence are equal.

\section{ReFERENCES to ApPendix A}

[Coa] J. Coates, p-adic L-functions and Iwasawa's theory, Algebraic Number Fields (A. Fröhlich, ed.), Academic Press, London, 1977, pp. 269-353. MR 57:276

[DR] P. Deligne and K. Ribet, Values of Abelian L-functions at negative integers over totally real fields, Invent. Math. 59 (1980), 227-286. MR 81m:12019

[Fed] L. J. Federer, Regulators, Iwasawa modules, and the Main Conjecture for $p=2$, Number Theory Related to Fermat's Last Theorem (N. Koblitz, ed.), Birkhäuser, Basel, 1982, pp. 289-296. MR 84a:10004

[Gr] R. Greenberg, On p-adic L-functions and cyclotomic fields, Nagoya Math. J. 56 (1974), 61-77. MR 50:12984

[Gr2] R. Greenberg, On p-adic L-functions and cyclotomic fields. II, Nagoya Math. J. 67 (1977), 139-158. MR 56:2964

[Gr3] R. Greenberg, On p-adic Artin L-functions, Nagoya Math. J. 89 (1983), 77-87. MR 85b:11104

[Gri] C. Greither, Class Groups of Abelian Fields, and the Main Conjecture, Ann. Inst. Fourier, Grenoble 42 (1992), 449-499. MR 93j:11071

[Iwa] K. Iwasawa, Lectures on p-adic L-functions, Princeton University Press, Princeton, 1972. MR 50:12974

[Iwa2] K. Iwasawa, On $\mathbb{Z}_{l}$-extensions of algebraic number fields, Annals of Math. 98 (1973), 246-326. MR 50:2120

[Ko] M. Kolster, A relation between the 2-primary parts of the Main Conjecture and the BirchTate Conjecture, Can. Bull. Math. 32 (1989), 248-251. MR 90k:11154

$[\mathrm{KNF}]$ M. Kolster, T. Nguyen Quang Do and V. Fleckinger, Twisted S-units, -adic class number formulas and the Lichtenbaum Conjectures, Duke Math. J. 84 (1996), 679-717. MR 97g:11136; MR 98k:11172

[Li] S. Lichtenbaum, On the values of zeta and L-functions: I, Annals of Math. 96 (1972), 338-360. MR 50:12975

$[\mathrm{MW}]$ B. Mazur and A. Wiles, Class-fields of Abelian extensions of $\mathbb{Q}$, Invent. Math. 76 (1984), 179-330. MR 85m:11069

[Ngu] T. Nguyen Quang Do, Une Étude Cohomologique de la Partie 2-Primaire de $K_{2} \mathcal{O}, K$ Theory 3 (1990), 523-542. MR 91h:11130

[Ws] L. C. Washington, Introduction to cyclotomic fields, Graduate Texts in Mathematics, vol. 83, Springer, 1982. MR 85g:11001

[Wi] A. Wiles, The Iwasawa conjecture for totally real fields, Annals of Math. 131 (1990), 493-540. MR 91i: 11163

[Z] T. Zink, Étale cohomology and duality in algebraic number fields, Appendix 2, Galois cohomology of algebraic number fields, by K. Haberland, Deutscher Verlag der Wissenschaften, Berlin, 1978. MR 81i:12009 


\section{Appendix B: Bloch and Lichtenbaum's spectral Sequence WITH COEFFICIENTS \\ By J. Rognes And C. Weibel}

In this appendix we prove that there is a "Bloch-Lichtenbaum spectral sequence" with finite coefficients $\mathbb{Z} / m$. We use this result in a crucial way in the main body of the paper, and several other papers have also cited the existence of such a spectral sequence, including [V2], [W3], and [K2].

To state the result, we define $C H^{i}(F, n ; \mathbb{Z} / m)$ to be the $n$th homotopy group of the simplicial Abelian group $[p] \mapsto \mathcal{Z}_{\mathrm{Bl}}^{i}(F, p) \otimes \mathbb{Z} / m=\mathcal{Z}_{\mathrm{Bl}}^{i}(F, p ; \mathbb{Z} / m)$.

Theorem B.1. For every integer $m$ and every field $F$ there is a convergent third quadrant spectral sequence

$$
E_{2}^{p, q}=C H^{-q}(F,-p-q ; \mathbb{Z} / m) \Longrightarrow K_{-p-q}(F ; \mathbb{Z} / m) .
$$

Here $E_{2}^{p, q}=0$ unless $q \leq p \leq 0$.

Before proving Theorem B.1, it is instructive to check how much of the proof in [BL] goes through if we replace all occurrences of the spectra $K(X)$ and $K H(X)$ by the corresponding spectra $K(X ; \mathbb{Z} / m)$ and $K H(X ; \mathbb{Z} / m)$. It turns out that almost the entire proof in $[\mathrm{BL}]$ goes through - everything except for the proof of the "key point."

The reason that Theorem B.1 is not an immediate consequence of the methods in [BL] is this: if $R$ is a regular ring and $I$ is a radical ideal in $R$, then $K_{0}(R, I)=0$ but $K_{0}(R, I ; \mathbb{Z} / m)$ can be nonzero, because there may be torsion in $K_{-1}(R, I)=$ $K_{0}(R / I) / K_{0}(R)$. Thus Corollary 2.3.3 of [BL] is false with finite coefficients. That is unfortunately the result which plays a crucial role in the proof of the "key point," Proposition 6.1 of $[\mathrm{BL}]$.

Here is the analog of [BL, (1.3.2)]. We will not explain the notation, which is identical to that of $[\mathrm{BL}]$, because it is not important for this appendix.

Proposition B.2. For every field $F$ and integers $i, p$ the spectrum map

$$
f: K_{\mathcal{V}^{i+1}}\left(\Delta^{p}, \Sigma\right) \rightarrow K_{\mathcal{V}^{i}}\left(\Delta^{p}, \Sigma\right)
$$

is null homotopic. Since $j i: K_{\mathcal{V}^{i+1}}\left(\Delta^{p}, \partial\right) \rightarrow K_{\mathcal{V}^{i}}\left(\Delta^{p}, \Sigma\right)$ and $i k: K_{\mathcal{V}^{i+1}}\left(\Delta^{p}, \Sigma\right) \rightarrow$ $K_{\mathcal{V}^{i}}\left(\Delta^{p-1}, \partial\right)$ factor through this map, it follows that for every integer $m$ there is a chain complex:

$$
\begin{aligned}
\cdots K_{0, \mathcal{V}^{i+1}}\left(\Delta^{p}, \partial ; \mathbb{Z} / m\right) \stackrel{i}{\rightarrow} K_{0, \mathcal{V}^{i}}\left(\Delta^{p}, \partial ; \mathbb{Z} / m\right) \stackrel{j}{\rightarrow} K_{0, \mathcal{V}^{i}}\left(\Delta^{p}, \Sigma ; \mathbb{Z} / m\right) \stackrel{k}{\rightarrow} \\
\stackrel{k}{\rightarrow} K_{0, \mathcal{V}^{i}}\left(\Delta^{p-1}, \partial ; \mathbb{Z} / m\right) \stackrel{i}{\rightarrow} K_{0, \mathcal{V}^{i-1}}\left(\Delta^{p-1}, \partial ; \mathbb{Z} / m\right) \cdots .
\end{aligned}
$$

Proof. We only need to promote the argument in [BL, 1.3] to the level of spectra. It suffices to show that the maps $f: K_{\mathcal{V}^{i+1}}\left(\Delta^{p}, \Sigma\right) \rightarrow K_{\mathcal{V}^{i}}\left(\Delta^{p}, \Sigma\right)$ are null homotopic, for then we can form the diagram of topological spaces analogous to diagram (1.3.1) of [BL], and take homotopy groups with any coefficients to get the chain complex (B.3).

By the Dold-Kan decomposition of the simplicial spectra $[p] \mapsto K_{\mathcal{V}^{i}}\left(\Delta^{p}\right)$ and $[p] \mapsto K_{\mathcal{V}^{i+1}}\left(\Delta^{p}\right)$, the multirelative map $f$ factors through the absolute map

$$
K_{\mathcal{V}^{i+1}}\left(\Delta^{p}\right) \rightarrow K_{\mathcal{V}^{i}}\left(\Delta^{p}\right)
$$

this is essentially the argument of [BL, 1.2.1]. In turn, the absolute map is a filtered colimit of maps $f_{W}: K_{W}\left(\Delta^{p}\right) \rightarrow K_{\mathcal{V}^{i}}\left(\Delta^{p}\right)$, so it suffices to see that the maps $f_{W}$ 
are null homotopic. If $R$ is such that $W=\operatorname{Spec}(R)$, then the use of "Quillen's trick" in [BL, 1.2.2] shows that $f_{W}$ factors through the map $K^{\prime}(R) \rightarrow K^{\prime}(R[t])$. But this map is null homotopic, as Quillen showed in [Q2] using the additivity theorem in $K$-theory.

We remark that Quillen's trick is also used in the discussion in 2.4, the proof of Proposition 5.2, and the start of the proof of Proposition 6.1 in [BL]. Each time the analog with finite coefficients is valid.

The notation in (B.3) is awkward. To simplify things, we shall write $K_{j}(i, p)$ for $K_{j, \mathcal{V}^{i}}\left(\Delta^{p}, \partial\right)$ and $K_{j}(i, p ; \mathbb{Z} / m)$ for $K_{j, \mathcal{V}^{i}}\left(\Delta^{p}, \partial ; \mathbb{Z} / m\right)$. We shall also write $\mathcal{Z}_{\mathrm{Bl}}^{i}(p)$ for $\mathcal{Z}_{\mathrm{Bl}}^{i}(F, p)_{\text {norm }}$ and $\mathcal{Z}_{\mathrm{Bl}}^{i}(p ; \mathbb{Z} / m)$ for $\mathcal{Z}_{\mathrm{Bl}}^{i}(F, p ; \mathbb{Z} / m)_{\text {norm }}$. With this notation, Theorem 1.3 .3 of $[\mathrm{BL}]$ says that the following sequence is exact:

(B.4) $\cdots K_{0}(i+1, p) \stackrel{i}{\rightarrow} K_{0}(i, p) \stackrel{j}{\rightarrow} \mathcal{Z}_{\mathrm{Bl}}^{i}(p) \stackrel{k}{\rightarrow} K_{0}(i, p-1) \stackrel{i}{\rightarrow} K_{0}(i-1, p-1) \cdots$.

Lemma B.5. If $y \in K_{0}(i, p)$ satisfies $m y=0$, then $y=i(x)$ for an $x \in K_{0}(i+1, p)$.

Proof. Since $\mathcal{Z}_{\mathrm{Bl}}^{i}(F, p)$ is a free Abelian group, its subgroup $\mathcal{Z}_{\mathrm{Bl}}^{i}(p)$ is torsion free. Hence we must have $j(y)=0$. Hence $y$ lifts by exactness of (B.4).

Here is the finite coefficient analog of Theorem 1.3.3 in [BL]. The proof of this result will occupy most of this appendix.

Theorem B.6. The sequences in (B.3) are exact for every field $F$.

To connect this result to Theorem B.1, we make the following observation. Because $K_{-1, \mathcal{V}^{i}}\left(\Delta^{p}\right)=0$, the identifications in [BL, Lemma 1.2.3] of $K_{0, \mathcal{V}^{i}}\left(\Delta^{p}\right)$ with $\mathcal{Z}_{\mathrm{Bl}}^{i}(F, p)$ and $K_{0, \mathcal{V}^{i}}\left(\Delta^{p}, \Sigma\right)$ with $\mathcal{Z}_{\mathrm{Bl}}^{i}(p)=\mathcal{Z}_{\mathrm{Bl}}^{i}(F, p)_{\text {norm }}$ become $K_{0, \mathcal{V}^{i}}\left(\Delta^{p} ; \mathbb{Z} / m\right) \cong$ $\mathcal{Z}_{\mathrm{Bl}}^{i}(F, p) \otimes \mathbb{Z} / m=\mathcal{Z}_{\mathrm{Bl}}^{i}(F, p ; \mathbb{Z} / m)$, and

$$
K_{0, \mathcal{V}^{i}}\left(\Delta^{p}, \Sigma ; \mathbb{Z} / m\right) \cong \mathcal{Z}_{\mathrm{Bl}}^{i}(p ; \mathbb{Z} / m)=\mathcal{Z}_{\mathrm{Bl}}^{i}(F, p ; \mathbb{Z} / m)_{\text {norm }} .
$$

The latter term occurs in the chain complex (B.3), and the finite coefficient analog of diagram [BL, (1.3.1)] shows that each sequence in (B.3) is exact at this term.

Proof of Theorem B.1, assuming Theorem B.6. We argue as in [BL, 1.3.4]. Given the above identification, the sequences in (B.3) assemble to form an exact couple with

$$
E_{1}^{-p,-q}=\mathcal{Z}_{\mathrm{Bl}}^{q}(F, p+q ; \mathbb{Z} / m)_{\text {norm }}
$$

and $D_{1}^{-p,-q}=K_{0, \mathcal{V}^{q}}\left(\Delta^{p+q}, \partial ; \mathbb{Z} / m\right)$. By inspection, $E_{2}^{-p,-q}=C H^{q}(F, p+q ; \mathbb{Z} / m)$. For convergence, we make some observations. If $q>0$, the notation clearly implies that $E_{1}^{p q}=D_{1}^{p q}=0$. If $p>0$, then $E_{1}^{p q}=0$ and $D_{1}^{p q} \cong K_{-p-q}(F ; \mathbb{Z} / m)$. Thus the exact couple lies in the third quadrant and converges to $K_{-p-q}(F ; \mathbb{Z} / m)$.

We now begin the proof of Theorem B.6. The following commutative diagram is a continuation to the right of the diagram (1.3.1) in [BL], and has exact rows. The vertical map $\mathcal{Z}_{\mathrm{Bl}}^{i+1}(p) \rightarrow \mathcal{Z}_{\mathrm{Bl}}^{i}(p)$ is zero by [BL, 1.2.2].




The diagonal map $h$ makes both triangles commute and is injective, by surjectivity of the connecting map $g$ and exactness of (B.4) at $K_{0}(i+1, p-1)$. From the universal coefficient formulas we have a diagram



Let us write ${ }_{m} \tilde{K}_{-1}(i+1, p)$ for the subgroup of all elements $\bar{x} \in K_{-1}(i+1, p)$ such that $m \bar{x}=i^{\prime}(\bar{x})=0$. Then the maps $i$ and $j$ induce maps $\bar{\imath}:{ }_{m} \tilde{K}_{-1}(i+1, p) \rightarrow$ $K_{0}(i, p) / m K_{0}(i, p)+i K_{0}(i+1, p)$ and $\bar{\jmath}:{ }_{m} K_{-1}(i, p) \rightarrow \mathcal{Z}_{\mathrm{Bl}}^{i}(p) / m \mathcal{Z}_{\mathrm{Bl}}^{i}(p)+j K_{0}(i, p)$.

Lemma B.8. The map $\bar{\jmath}$ induces an injection

$$
\frac{{ }_{m} K_{-1}(i, p)}{i^{\prime}\left({ }_{m} K_{-1}(i+1, p)\right)} \rightarrow \frac{\mathcal{Z}_{\mathrm{Bl}}^{i}(p)}{m \mathcal{Z}_{\mathrm{Bl}}^{i}(p)+j K_{0}(i, p)} .
$$

Proof. Let $\bar{y} \in K_{-1}(i, p)$ be the image of an element $\gamma \in K_{0}(i, p ; \mathbb{Z} / m)$, and let $y \in K_{0}(i, p-1)$ be a lift of $\bar{y}$ in (B.7). Since $m \bar{y}=0$, there is a $z \in \mathcal{Z}_{\mathrm{Bl}}^{i}(p)$ with $k(z)=m y$. Since the bottom row of (B.7) is a fibration sequence for $j$, it is well known from topology that the class of $z$ represents $j(\gamma)$ in $\mathcal{Z}_{\mathrm{Bl}}^{i}(p) / m \mathcal{Z}_{\mathrm{Bl}}^{i}(p)+$ $j K_{0}(i, p)$ up to sign. If $z=m t+j(u)$, then $y^{\prime}=y-k(t)$ is another lift of $\bar{y}$ with $m y^{\prime}=0$. By Lemma B.5, $y^{\prime}=i(x)$ for some $x$. By commutativity of the right square in (B.7), we have $\bar{y}=i^{\prime}(\bar{x})$ for some $\bar{x}$. Finally $m \bar{x}=0$ by injectivity of $h$.

Definition B.9. Given an element $\bar{x}$ of $K_{-1}(i+1, p)$ such that $m \bar{x}=i^{\prime}(\bar{x})=0$, we define an element $a$ in $K_{0}(i, p) / m K_{0}(i, p)+i K_{0}(i+1, p)$ as follows. Let $y=$ $h(\bar{x}) \in K_{0}(i, p-1)$. Then $y=i(x)$ for any lift $x \in K_{0}(i+1, p-1)$ of $\bar{x}$. Using (B.7) choose a lift $z \in \mathcal{Z}_{\mathrm{Bl}}^{i}(p)$ of $y$. Since $m \bar{x}=0$ we have $m y=0$, and we define $a$ to be a lift of $m z$. A diagram chase on (B.7) shows that $a$ is well defined modulo $m K_{0}(i, p)+i K_{0}(i+1, p)$. This yields a map

$$
\phi:{ }_{m} \tilde{K}_{-1}(i+1, p) \rightarrow K_{0}(i, p) / m K_{0}(i, p)+i K_{0}(i+1, p) .
$$

An analysis of the topological spaces involved shows that the map $\phi$ agrees up to sign with the map $\bar{\imath}$ induced by $i: K_{0}(i+1, p ; \mathbb{Z} / m) \rightarrow K_{0}(i, p ; \mathbb{Z} / m)$.

Lemma B.10. The map $\phi$ is an injection.

Proof. Suppose that $a=m b+i(c)$ for some $b$ and $c$. Then $m j(b)=j(a)=m z$. Since $\mathcal{Z}_{\mathrm{Bl}}^{i}(p)$ is torsion free, this yields $j(b)=z$. Hence $y=k j(b)=0$. Since $i(x)=0$, exactness of (B.4) shows that $x=k\left(z^{\prime}\right)$ and hence $\bar{x}=0$.

Exactness of (B.3) at $K_{0}(i, p ; \mathbb{Z} / m)$. Let $\gamma \in K_{0}(i, p ; \mathbb{Z} / m)$ be in the kernel of $j$. By Lemma B.8, $\gamma$ comes from an element $a \in K_{0}(i, p)$ with $j(a)=m z$ for some $z \in \mathcal{Z}_{\mathrm{Bl}}^{i}(p)$. Set $y=k(z)$. Since $m y=k j(a)=0$, Lemma B.5 implies that $y=i(x)$ for some $x \in K_{0}(i+1, p-1)$. If $\bar{x}$ denotes the image of $x$ in $K_{-1}(i+1, p)$, Definition B.9 shows that $a=\phi(\bar{x})$. 
Exactness of (B.3) at $K_{0}(i, p-1 ; \mathbb{Z} / m)$. Let $\gamma \in K_{0}(i, p-1 ; \mathbb{Z} / m)$ be in the kernel of $i$. By Lemma B.10, $\gamma$ comes from an element $x$ of $K_{0}(i, p-1)$ with $i(x)=m y$ for some $y \in K_{0}(i-1, p-1)$. Hence we have $m j(y)=j i(x)=0$. Because $\mathcal{Z}_{\mathrm{Bl}}^{i}(p)$ is torsion free this implies that $j(y)=0$, and hence that $y=i(w)$ for some $w \in K_{0}(i, p-1)$. Hence $i(x-m w)=0$, so exactness of (B.4) implies that $x-m w=k(z)$ for some $z \in \mathcal{Z}_{\mathrm{Bl}}^{i}(p)$. Hence $\gamma$ is the image of $z$.

This finishes the proof of Theorem B.6, and hence the proof of Theorem B.1.

\section{REFERENCES}

[A] J. F. Adams, On the groups $J(X) . I V$, Topology 5 (1966), 21-71. MR 33:6628

[Bl] S. Bloch, Algebraic cycles and higher K-theory, Adv. Math. 61 (1986), 267-304. MR 88f: 18010

[BL] S. Bloch and S. Lichtenbaum, A spectral sequence for motivic cohomology, Invent. Math. (to appear).

[Bo] A. Borel, Stable real cohomology of arithmetic groups, Ann. Sci. Ecole Norm. Sup. 7 (1974), 235-272. MR 52:8338

[CF] J. W. S. Cassels and A. Frölich, eds., Algebraic Number Theory, Academic Press, 1967. MR 35:6500

[DF] W. Dwyer and E. Friedlander, Algebraic and étale K-theory, Trans. AMS 292 (1985), 247280. MR 87h: 18013

[FV] E. Friedlander and V. Voevodsky, Bivariant cycle cohomology, UIUC K-theory preprint server, no. 75, 1995.

[Ga] O. Gabber, K-theory of Henselian local rings and Henselian pairs, AMS Contemp. Math., vol. 126, 1992, pp. 59-70. MR 93c:19005

[Gri] C. Greither, Class groups of Abelian fields, and the Main Conjecture, Ann. Inst. Fourier, Grenoble 42 (1992), 449-499. MR 93j:11071

[HS] B. Harris and G. Segal, $K_{i}$ groups of rings of algebraic integers, Annals of Math. 101 (1975), 20-33. MR 52:8222

[H] R. T. Hoobler, When is $\operatorname{Br}(X)=\operatorname{Br}^{\prime}(X)$ ?, Lecture Notes in Math., vol. 917, Springer Verlag, 1982, pp. 231-244. MR 83g:14006

[J] U. Jannsen, Continuous étale cohomology, Math. Ann. 280 (1988), 207-245. MR 89a:14022

[K1] B. Kahn, Some conjectures on the algebraic K-theory of fields I: K-theory with coefficients and étale cohomology, Algebraic $K$-theory: Connections with Geometry and Topology ( $\mathrm{J}$. F. Jardine and V. P. Snaith, eds.), NATO ASI Series C, vol. 279, Kluwer, 1989, pp. 117-176. MR 91d:19006

[K2] _ The Quillen-Lichtenbaum Conjecture at the prime 2, UIUC K-theory preprint server, no. 208, 1997.

[L1] S. Lichtenbaum, On the values of zeta and L-functions, I, Annals of Math. 96 (1972), 338-360. MR 50:12975

[L2] - Values of zeta functions, étale cohomology, and algebraic K-theory, Lecture Notes in Math., vol. 342, Springer Verlag, 1973, pp. 489-501. MR 53:10765

[M1] J. S. Milne, Étale Cohomology, Princeton University Press, 1980. MR 81j:14002

[M2] - Arithmetic Duality Theorems, Academic Press, 1986. MR 88e:14028

[NS] Yu. P. Nesterenko and A. A. Suslin, Homology of the general linear group over a local ring, and Milnor's K-theory (Russian), Izv. Akad. Nauk. SSSR Ser. Mat. 53 (1989), 121-146. MR 90a:20092

[N] J. Neukirch, Class Field Theory, Grundlehren der mathematischen Wissenschaften, vol. 280, Springer Verlag, 1986. MR 87i: 11005

[P] I. Panin, On a theorem of Hurewicz and $K$-theory of complete discrete valuation rings, Math. USSR Izvestiya 29 (1987), 119-131. MR 88a:18021

[Q1] D. Quillen, On the cohomology and $K$-theory of the general linear group over a finite field, Annals of Math. 96 (1972), 552-586. MR 47:3565

[Q2] - Higher algebraic K-theory, I, Lecture Notes in Math., vol. 341, Springer Verlag, 1973, pp. 85-147. MR 49:2895

[Q3] - Finite generation of the groups $K_{i}$ of rings of algebraic integers, Lecture Notes in Math., vol. 341, Springer Verlag, 1973, pp. 179-198. MR 50:2305 
[Q4] Higher algebraic K-theory, Proc. Intern. Congress Math., Vancouver, 1974, vol. I, Canad. Math. Soc., 1975, pp. 171-176. MR 54:10382

[Q5] Letter from Quillen to Milnor on $\operatorname{Im}\left(\pi_{i} O \rightarrow \pi_{i}^{S} \rightarrow K_{i} \mathbb{Z}\right)$, Lecture Notes in Math., vol. 551, Springer Verlag, 1976, pp. 182-188. MR 58:2811

[R] J. Rognes, Algebraic K-theory of the two-adic integers, J. Pure Appl. Algebra 134 (1999), 219-286. CMP 99:06

[RO] J. Rognes and P. A. Østvær, Two-primary algebraic K-theory of two-regular number fields, Math. Z. (to appear).

[RW] J. Rognes and C. Weibel, Étale descent for two-primary algebraic K-theory of totally imaginary number fields, K-Theory 16 (1999), 101-104. CMP 99:08

[Sc] P. Schneider, Über gewisse Galoiscohomologiegruppen, Math. Z. 168 (1979), 181-205. MR 81i: 12010

[Se] J.-P. Serre, Local Fields, Graduate Texts in Mathematics, vol. 67, Springer Verlag, 1979. MR 82e: 12016

[So] C. Soulé, $K$-théorie des anneaux d'entiers de corps de nombres et cohomologie étale, Invent. Math. 55 (1979), 251-295. MR 81i: 12016

[S1] A. A. Suslin, On the K-theory of algebraically closed fields, Invent. Math. 73 (1983), 241245. MR 85h:18008a

[S2] Higher Chow groups and étale cohomology, Preprint, 1994.

[S3] _ Algebraic K-theory and motivic cohomology, Proc. Intern. Congress Math., Zürich, 1994, Birkhäuser, 1995, pp. 342-351. MR 98g:19007

[SV] A. A. Suslin and V. Voevodsky, The Bloch-Kato conjecture and motivic cohomology with finite coefficients, UIUC K-theory preprint server, no. 83, 1995.

[T] J. Tate, Duality theorems in Galois cohomology over number fields, Proc. Intern. Congress Math., Stockholm, 1962, Inst. Mittag-Leffler, 1963, pp. 234-241. MR 31:168

[V1] V. Voevodsky, Triangulated categories of motives over a field, UIUC K-theory preprint server, no. 74, 1995.

[V2] _ The Milnor Conjecture, UIUC K-theory preprint server, no. 170, 1996.

$[\mathrm{Wg}]$ J. B. Wagoner, Continuous cohomology and p-adic $K$-theory, Lecture Notes in Math., vol. 551, Springer Verlag, 1976, pp. 241-248. MR 58:16609

[Ws] L. C. Washington, Introduction to Cyclotomic Fields, Graduate Texts in Mathematics, vol. 83, Springer Verlag, 1982. MR 85g:11001

[W1] C. Weibel, Étale Chern classes at the prime 2, Algebraic K-theory and Algebraic Topology (P. Goerss and J. F. Jardine, eds.), NATO ASI Series C, vol. 407, Kluwer, 1993, pp. 249-286. MR 96m:19013

[W2] _ An introduction to homological algebra, Cambridge Univ. Press, 1994. MR 95f: 18001

[W3] - The 2-torsion in the K-theory of the integers, C. R. Acad. Sci. Paris 324 (1997), 615-620. MR 98h:19001

[Wi] A. Wiles, The Iwasawa Conjecture for totally real fields, Annals of Math. 131 (1990), 493-540. MR 91i: 11163

Department of Mathematics, University of Oslo, Oslo, Norway

E-mail address: rognes@math.uio.no

Department of Mathematics, Rutgers University, New Brunswick, New Jersey 089032101

E-mail address: weibel@math.rutgers.edu

Department of Mathematics and Statistics, McMaster University, Hamilton, OnTARIO, CANADA L8S $4 \mathrm{~K} 1$

E-mail address: kolster@mcmail.CIS.McMaster.CA 